\title{
Ultrafast optical manipulation of magnetic order
}

\author{
Andrei Kirilyuk, ${ }^{*}$ Alexey V. Kimel, and Theo Rasing \\ Radboud University Nijmegen, Institute for Molecules and Materials, Heyendaalseweg \\ 135, 6525 AJ Nijmegen, The Netherlands
}

(Published 22 September 2010)

\begin{abstract}
The interaction of subpicosecond laser pulses with magnetically ordered materials has developed into a fascinating research topic in modern magnetism. From the discovery of subpicosecond demagnetization over a decade ago to the recent demonstration of magnetization reversal by a single $40 \mathrm{fs}$ laser pulse, the manipulation of magnetic order by ultrashort laser pulses has become a fundamentally challenging topic with a potentially high impact for future spintronics, data storage and manipulation, and quantum computation. Understanding the underlying mechanisms implies understanding the interaction of photons with charges, spins, and lattice, and the angular momentum transfer between them. This paper will review the progress in this field of laser manipulation of magnetic order in a systematic way. Starting with a historical introduction, the interaction of light with magnetically ordered matter is discussed. By investigating metals, semiconductors, and dielectrics, the roles of (nearly) free electrons, charge redistributions, and spin-orbit and spin-lattice interactions can partly be separated, and effects due to heating can be distinguished from those that are not. It will be shown that there is a fundamental distinction between processes that involve the actual absorption of photons and those that do not. It turns out that for the latter, the polarization of light plays an essential role in the manipulation of the magnetic moments at the femtosecond time scale. Thus, circularly and linearly polarized pulses are shown to act as strong transient magnetic field pulses originating from the nonabsorptive inverse Faraday and inverse Cotton-Mouton effects, respectively. The recent progress in the understanding of magneto-optical effects on the femtosecond time scale together with the mentioned inverse, optomagnetic effects promises a bright future for this field of ultrafast optical manipulation of magnetic order or femtomagnetism.
\end{abstract}

DOI: 10.1103/RevModPhys.82.2731 PACS number(s): 75.78.Jp, 85.75.-d, 78.47.jh, 75.60.-d

\section{CONTENTS}

I. Introduction

A. Issues and challenges in magnetization dynamics

B. The problem of ultrafast laser control of magnetic order

II. Theoretical Considerations

A. Dynamics of magnetic moments: Landau-Lifshitz-Gilbert equation

B. Finite temperature: Landau-Lifshitz-Bloch equation

C. Interaction of photons and spins

III. Experimental Techniques

A. Pump-and-probe method

B. Optical probe

C. Ultraviolet probe and spin-polarized electrons

D. Far-infrared probe

E. X-ray probe

IV. Thermal Effects of Laser Excitation

A. Ultrafast demagnetization of metallic ferromagnets

1. Experimental observation of ultrafast demagnetization

2732

2732

2733

2734

2734

2735

2735

2737

2737

2737

2738

2739

2739

2739

2739

2. Phenomenological three-temperature model

3. Spin-flip and angular momentum transfer in metals

4. Interaction among charge, lattice, and spin subsystems

\footnotetext{
*a.kirilyuk@science.ru.nl
}

a. Time-resolved electron photoemission experiments

b. Time-resolved x-ray experiments 2745

5. Microscopic models of ultrafast demagnetization

2746

B. Demagnetization of magnetic semiconductors 2747

C. Demagnetization of magnetic dielectrics 2748

D. Demagnetization of magnetic half metals 2749

E. Laser-induced coherent magnetic precession 2750

1. Precession in exchange-biased bilayers 2750

2. Precession in nanostructures 2751

3. Precession in (III,Mn)As ferromagnetic semiconductors

4. Precession in ferrimagnetic materials

F. Laser-induced phase transitions between two magnetic states

1. Spin reorientation in $\mathrm{TmFeO}_{3}$

2755

2. Spin reorientation in $(\mathrm{Ga}, \mathrm{Mn}) \mathrm{As}$

2756

3. AM-to-FM phase transition in FeRh 2756

G. Magnetization reversal

2757

V. Nonthermal Photomagnetic Effects

2758

A. Photomagnetic modification of magnetocrystalline anisotropy

1. Laser-induced precession in magnetic garnet films

a. Experimental observations 2758

b. Phenomenological model 2759

c. Microscopic mechanism

2760

2. Laser-induced magnetic anisotropy in antiferromagnetic $\mathrm{NiO}$

2761 
3. Photomagnetic excitation of spin precession in $(\mathrm{Ga}, \mathrm{Mn}) \mathrm{As}$

B. Light-enhanced magnetization in (III,Mn)As semiconductors

VI. Nonthermal Optomagnetic Effects

A. Inverse magneto-optical excitation of magnetization dynamics: Theory

1. Formal theory of inverse optomagnetic effects

2. Example I: Cubic ferromagnet

3. Example II: Two-sublattice antiferromagnet

B. Excitation of precessional magnetization dynamics with circularly polarized light

1. Optical excitation of magnetic precession in garnets

2. Optical excitation of antiferromagnetic resonance in $\mathrm{DyFeO}_{3}$

3. Optical excitation of precession in $\mathrm{GdFeCo}$

C. All-optical control and switching

1. Double-pump coherent control of magnetic precession

2. Femtosecond switching in magnetic garnets

3. Inertia-driven switching in antiferromagnets

4. All-optical magnetization reversal

5. Reversal mechanism via a nonequilibrium state

D. Excitation of the magnetization dynamics with linearly polarized light

1. Detection of the FMR mode via magnetic linear birefringence in $\mathrm{FeBO}_{3}$

2. Excitation of coherent magnons by linearly and circularly polarized pump pulses

3. ISRS as the mechanism of coherent magnon excitation

4. Effective light-induced field approach

VII. Conclusions and Outlook

Acknowledgments

References

\section{INTRODUCTION}

\section{A. Issues and challenges in magnetization dynamics}

The time scale for magnetization dynamics is extremely long and varies from the billions of years connected to geological events such as the reversal of the magnetic poles down to the femtosecond regime connected with the exchange interaction between spins. From a more practical point of view, the demands for the ever-increasing speed of storage of information in magnetic media plus the intrinsic limitations that are connected with the generation of magnetic field pulses by current have triggered intense searches for ways to control magnetization by means other than magnetic fields. Since the demonstration of subpicosecond demagnetization by a $60 \mathrm{fs}$ laser pulse by Beaurepaire et al. (1996), manipulating and controlling magnetization with ultrashort laser pulses has become a challenge. Femtosecond laser pulses offer the intriguing possibility to probe a magnetic system on a time scale that

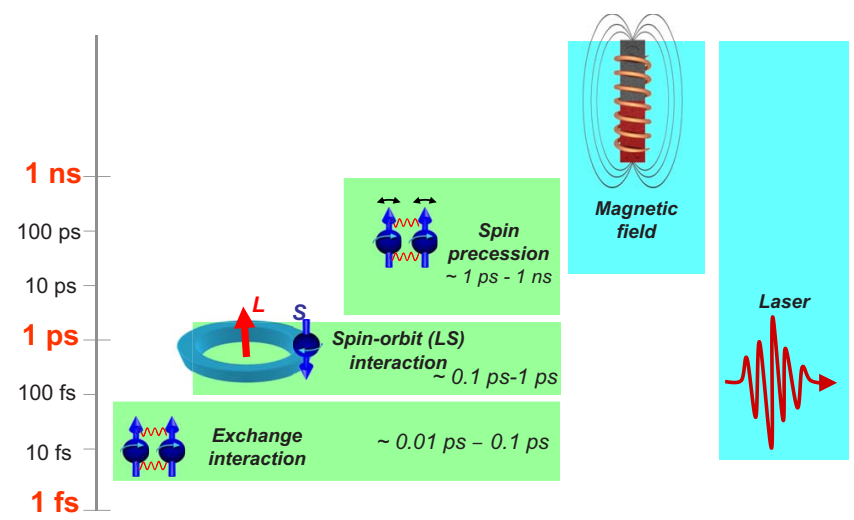

FIG. 1. (Color online) Time scales in magnetism as compared to magnetic field and laser pulses. The short duration of the laser pulses makes them an attractive alternative to manipulate the magnetization.

corresponds to the (equilibrium) exchange interaction, responsible for the existence of magnetic order, while being much faster than the time scale of spin-orbit interaction (1-10 ps) or magnetic precession (100-1000 ps); see Fig. 1. Because the latter is considered to set the limiting time scale for magnetization reversal, the option of femtosecond optical excitation immediately leads to the question whether it would be possible to reverse magnetization faster than within half a precessional period. As magnetism is intimately connected to angular momentum, this question can be rephrased in terms of the more fundamental issues of conservation and transfer of angular momentum: How fast and between which reservoirs can angular momentum be exchanged and is this even possible on time scales shorter than that of the spin-orbit interaction?

While such questions are not relevant at longer times and for equilibrium states, they become increasingly important as times become shorter and, one by one, the various reservoirs of a magnetic system, such as the magnetically ordered spins, the electron system, and the lattice, become dynamically isolated. The field of ultrafast magnetization dynamics is therefore concerned with the investigation of the changes in a magnetic system as energy and angular momentum are exchanged between the thermodynamic reservoirs of the system (Stöhr and Siegmann, 2006).

Although deeply fundamental in nature, such studies are also highly relevant for technological applications. Indeed, whereas electronic industry is successfully entering the nanoworld following Moore's law, the speed of manipulating and storing data lags behind, creating a so-called ultrafast technology gap. This is also evident in modern PCs that already have a clock speed of a few gigahertz while the storage on a magnetic hard disk requires a few nanoseconds. A similar problem is experienced by the emerging field of spintronics as in, for example, magnetic random access memory devices. Therefore, the study of the fundamental and practical limits on the speed of manipulation of the magnetization direction is obviously also of great importance for 
magnetic recording and information processing technologies.

In magnetic memory devices, logical bits ("ones" and "zeros") are stored by setting the magnetization vector of individual magnetic domains either "up" or "down." The conventional way to record a magnetic bit is to reverse the magnetization by applying a magnetic field (Landau and Lifshitz, 1984; Hillebrands and Ounadjela, 2002). Intuitively, one would expect that switching could be infinitely fast, limited only by the attainable strength and shortness of the magnetic field pulse. However, recent experiments on magnetization reversal using uniquely short and strong magnetic field pulses generated by relativistic electrons from the Stanford Linear Accelerator (Tudosa et al., 2004) suggest that there is a speed limit on such a switching. It was shown that deterministic magnetization reversal does not take place if the magnetic field pulse is shorter than 2 ps. Could optical pulses be an alternative?

\section{B. The problem of ultrafast laser control of magnetic order}

The discovery of ultrafast demagnetization of a $\mathrm{Ni}$ film by a $60 \mathrm{fs}$ optical laser pulse (Beaurepaire et al., 1996) triggered the new and booming field of ultrafast laser manipulation of magnetization. Subsequent experiments not only confirmed these findings (Hohlfeld et al., 1997; Scholl et al., 1997; Güdde et al., 1999; Ju et al., 1999; Koopmans et al., 2000; Bigot, 2001; Hicken, 2003; Rhie et al., 2003; Bigot et al., 2004; Ogasawara et al., 2005) but also demonstrated the possibility to optically generate coherent magnetic precession (Ju, Nurmikko, et al., 1998; van Kampen et al., 2002), laser-induced spin reorientation (Kimel, Kirilyuk, et al., 2004; Bigot et al., 2005), or even modification of the magnetic structure (Ju et al., 2004; Thiele et al., 2004) and this all on a time scale of 1 ps or less. However, despite all these exciting experimental results, the physics of ultrafast optical manipulation of magnetism is still poorly understood.

A closer look at this problem reveals that excitation with a femtosecond laser pulse puts a magnetic medium in a highly nonequilibrium state, where the conventional macrospin approximation fails and a description of magnetic phenomena in terms of thermodynamics is no longer valid. In the subpicosecond time domain, typical times are comparable to or shorter than the characteristic time of spin-orbit interaction, and the magnetic anisotropy becomes a time-dependent parameter. Note that, although the spin-orbit coupling is an important ingredient of the magnetic anisotropy mechanism, the latter is the result of a balance between different crystalfield-split states. Therefore, the typical anisotropy energy is considerably lower than that of spin-orbit coupling, which is also translated into the corresponding response times. At shorter time scales even the exchange interaction should be considered as time dependent. All these issues seriously complicate a theoretical analysis of this problem. In addition, experimental studies of ultrafast magnetization dynamics are often ham- pered by artifacts, and interpretations of the same data are often the subject of heated debates.

What are the roles of spin-orbit, spin-lattice, and electron-lattice interactions in the ultrafast optical control of magnetism? How does the electronic band structure affect the speed of the laser-induced magnetic changes? A systematic study of the laser-induced phenomena in a broad class of materials may answer these questions, as optical control of magnetic order has been demonstrated in metals, semiconductors, and dielectrics. So far several attempts to summarize and systematize these studies have been dedicated to metals (Bigot, 2001; Zhang, Hübner, et al., 2002; Hicken, 2003; Bennemann, 2004; Bovensiepen, 2007), ferromagnetic semiconductors (Wang et al., 2006), or dielectrics (Kirilyuk et al., 2006; Kimel et al., 2007).

This review aims to introduce and summarize the experimental and theoretical studies of ultrafast optical manipulation of spins in all the classes of both ferromagnetically and antiferromagnetically ordered solids studied so far, including metals, semiconductors, and dielectrics. We present an overview of the different experimental and theoretical approaches to the problem and distinguish effects of light on the net magnetization, magnetic anisotropy, and magnetic structure. As a result, important conclusions can be drawn about the role of different reservoirs of angular momentum (free electrons, orbital motion, and lattice) and their mutual exchange for the ultrafast optical manipulation of magnetism.

The effects of a pump laser pulse on a magnetic system could be classified as belonging to one of the following classes:

(1) Thermal effects: Because of the absorption of photons, energy is pumped into the medium. The change in the magnetization corresponds to that of spin temperature: $M=M\left(T_{s}\right)$. Since in the electric dipole approximation spin-flip transitions are forbidden, the direct pumping of energy from light to spins is not effective. Instead, light pumps the energy into the electron and phonon system. The time scale of the subsequent magnetization change is determined by internal equilibration processes such as electronelectron, electron-phonon, and electron-spin interactions, which for itinerant ferromagnets can be very short, down to $50 \mathrm{fs}$. For dielectric magnets, in contrast, this time is of the order of a nanosecond due to the absence of direct electron-spin processes. The lifetime of such thermal effects is given by external parameters such as thermal conductivity of a substrate as well as the geometry of the sample.

(2) Nonthermal (photomagnetic) effects involving the absorption of pump photons (Kabychenkov, 1991): In this case the photons are absorbed via certain electronic states that have a direct influence on magnetic parameters, such as, for example, the magnetocrystalline anisotropy. The change is instantaneous (e.g., the rise time of the pump pulse). These parameters, in turn, cause a motion of the magnetic mo- 
ments that obeys the usual precessional behavior. The lifetime of this effect is given by the lifetime of the corresponding electronic states. Note, however, that if this time is much shorter than the precession period, the effect will be difficult to detect.

(3) And finally, there are nonthermal optomagnetic effects that do not require the absorption of pump photons but are based on an optically coherent stimulated Raman scattering mechanism (Kabychenkov, 1991). The action of this mechanism can be considered as instantaneous and is limited by the spin-orbit coupling, which is the driving force behind the change in the magnetization in this case ( $\sim 20$ fs for a typical $50 \mathrm{meV}$ value of spin-orbit coupling). The lifetime of the effect coincides with that of optical coherence (100-200 fs). Note that in practice thermal effects are always present to some extent.

\section{THEORETICAL CONSIDERATIONS}

\section{A. Dynamics of magnetic moments: Landau-Lifshitz-Gilbert equation}

The interactions of magnetic moments with magnetic fields are basic to the understanding of all magnetic phenomena and may be applied in many ways. Homogeneously magnetized solids exhibit a magnetic moment, which for a volume $V$ is given by $\mathbf{m}=V \mathbf{M}$, where $\mathbf{M}$ is the magnetization. If $V$ is the atomic volume, then $\mathbf{m}$ is the magnetic moment per atom; if $V$ is the volume of the magnetic solid, $\mathbf{m}$ is the total magnetic moment of the body. The latter case is often called the "macrospin approximation." Also, for the inhomogeneous case, the magnetic solid can often be subdivided into small regions in which the magnetization can be assumed homogeneous. These regions are large enough that the motion of the magnetization can in most cases be described classically.

The precessional motion of a magnetic moment in the absence of damping is described by the torque equation. According to quantum theory, the angular momentum associated with a magnetic moment $\mathbf{m}$ is

$$
\mathbf{L}=\mathbf{m} / \gamma,
$$

where $\gamma$ is the gyromagnetic ratio. The torque on the magnetic moment $\mathbf{m}$ exerted by a magnetic field $\mathbf{H}$ is

$$
\mathbf{T}=\mathbf{m} \times \mathbf{H} .
$$

The change in angular momentum with time equals the torque:

$$
\frac{d \mathbf{L}}{d t}=\frac{d}{d t} \frac{\mathbf{m}}{\gamma}=\mathbf{m} \times \mathbf{H} .
$$

If the spins not only experience the action of the external magnetic field but are also affected by the magnetocrystalline anisotropy, shape anisotropy, magnetic dipole interaction, etc., the situation becomes more compli- cated. All these interactions will contribute to the thermodynamical potential $\Phi$, and the combined action of all these contributions can be considered as an effective magnetic field

$$
\mathbf{H}^{\mathrm{eff}}=-\partial \Phi / \partial \mathbf{M} \text {. }
$$

Thus the motion of the magnetization vector can be written as the following equation, named after Landau and Lifshitz (Landau and Lifshitz, 1935):

$$
d \mathbf{m} / d t=\gamma \mathbf{m} \times \mathbf{H}^{\mathrm{eff}},
$$

which describes the precession of the magnetic moment around the effective field $\mathbf{H}^{\mathrm{eff}}$. As mentioned, $\mathbf{H}^{\mathrm{eff}}$ contains many contributions:

$$
\mathbf{H}^{\mathrm{eff}}=\mathbf{H}_{\mathrm{ext}}+\mathbf{H}_{\mathrm{ani}}+\mathbf{H}_{\mathrm{dem}}+\cdots,
$$

where $\mathbf{H}_{\text {ext }}$ is the external applied field, $\mathbf{H}_{\text {ani }}$ is the anisotropy field, and $\mathbf{H}_{\mathrm{dem}}$ is the demagnetization field. Except for $\mathbf{H}_{\mathrm{ext}}$, all other contributions will be material dependent. Consequently, optical excitation of a magnetic material may result, via optically induced changes in the material-related fields, in a change in $\mathbf{H}^{\mathrm{eff}}$, giving rise to optically induced magnetization dynamics.

At equilibrium, the change in angular momentum with time is zero, and thus the torque is zero. A viscous damping term can be included to describe the motion of a precessing magnetic moment toward equilibrium. A dissipative term proportional to the generalized velocity $(-\partial \mathbf{m} / \partial t)$ is then added to the effective field. This dissipative term slows down the motion of the magnetic moment and eventually aligns $\mathbf{m}$ parallel to $\mathbf{H}^{\mathrm{eff}}$. This gives the Landau-Lifshitz-Gilbert (LLG) equation of motion (Gilbert, 1955):

$$
\frac{\partial \mathbf{m}}{\partial t}=\gamma \mathbf{m} \times \mathbf{H}^{\mathrm{eff}}+\frac{\alpha}{|\mathbf{m}|} \mathbf{m} \times \frac{\partial \mathbf{m}}{\partial t},
$$

where $\alpha$ is the dimensionless phenomenological Gilbert damping constant.

Equation (7) may be used to study the switching dynamics of small magnetic particles. If the particles are sufficiently small, the magnetization may be assumed to remain uniform during this reversal process, and the only contributions to the effective field are the anisotropy field, the demagnetizing field, and the applied external field. For larger samples, and in the case of inhomogeneous dynamics, such as spin waves with $k \neq 0$, the magnetic moment becomes a function of spatial coordinates: $\mathbf{m}=\mathbf{m}(\mathbf{r})$. The effective magnetic field in this case also acquires a contribution from the exchange interaction. In this case, nonhomogeneous elementary excitations of the magnetic medium may exist, first proposed by Bloch in 1930 (Bloch, 1930). These excitations are called spin waves and involve many lattice sites. More details on these aspects can be found in Hillebrands and Ounadjela (2002).

The LLG equation can also be used in the atomistic limit to calculate the evolution of the spin system using Langevin dynamics, which has proved to be a powerful 
approach to modeling ultrafast magnetization processes (Kazantseva, Nowak, et al., 2008; Atxitia et al., 2009a); see also the next section.

Some limitation of the LLG equation may come from the fact that, on a time scale shorter than the spin-orbit coupling (of the order of $20 \mathrm{fs}$ ), the description with a single gyromagnetic ratio fails and spin and orbital contributions must be considered separately.

\section{B. Finite temperature: Landau-Lifshitz-Bloch equation}

The Landau-Lifshitz-Gilbert equation considers magnetization as a vector of fixed length and ignores its longitudinal relaxation. Such an approach is obviously unsatisfactory at elevated temperatures since magnetization entering the LLG equation is an average over some distribution function and its magnitude can change. On the other hand, the atomistic approach described in the previous section is prohibitively time consuming in macroscopic systems.

A macroscopic equation of motion for the magnetization of a ferromagnet at elevated temperatures should thus contain both transverse and longitudinal relaxation terms and should interpolate between the LandauLifshitz equation at low temperatures and the Bloch equation (Bloch, 1946) at high temperatures.

We start with an atomistic approach where a magnetic atom is described as a classical spin vector $\mathbf{s}$ of unit length. The magnetic moment of the atom is given by $\mu=\mu_{0} \mathbf{s}$. In the case of a weak coupling with the bath, the dynamics of the vector $\mathbf{s}$ can be described with the help of the stochastic Landau-Lifshitz equation,

$$
\frac{d \mathbf{s}}{d t}=\gamma[\mathbf{s} \times(\mathbf{H}+\zeta)]-\gamma \lambda[\mathbf{s} \times[\mathbf{s} \times \mathbf{H}]],
$$

with $\lambda<0,|\lambda| \gg 1$, where correlators of the $\alpha, \beta=x, y, z$ components of the Langevin field $\zeta(t)$ are given by

$$
\left\langle\zeta_{\alpha}(t) \zeta_{\beta}\left(t^{\prime}\right)\right\rangle=\frac{2 \lambda T}{\gamma \mu_{0}} \delta_{\alpha \beta} \delta\left(t-t^{\prime}\right),
$$

with $\mu_{0}$ the atomic magnetic moment and $\lambda$ the parameter describing the coupling to the bath system. The coefficient in front of the delta function in Eq. (9) is determined by the fluctuation-dissipation theorem, which may limit the applicability of this approach on a time scale comparable to the correlation time of electron system, about 10 fs for metals (Stöhr and Siegmann, 2006).

The basis of Eq. (9) is thus the separation of time scales, assuming that the heat bath (phonon or electron system) acts much faster than the spin system. In this case, the bath degrees of freedom can be averaged out and replaced by a stochastic field with white noise correlation functions. The ensemble-averaged spin polarization gives the magnetization of the material: $\mathbf{m}$ $=\mu_{0}\langle\mathbf{s}\rangle$. Then, Eq. (8) results in the following LandauLifshitz-Bloch equation (Garanin, 1991, 1997):

$$
\begin{aligned}
\frac{d \mathbf{m}}{d t}= & \gamma\left[\mathbf{m} \times \mathbf{H}^{\mathrm{eff}}\right]-\gamma \lambda_{\|} \frac{\left(\mathbf{m} \cdot \mathbf{H}^{\mathrm{eff}}\right) \mathbf{m}}{m^{2}} \\
& +\gamma \lambda_{\perp} \frac{\left[\mathbf{m} \times\left[\mathbf{m} \times \mathbf{H}^{\mathrm{eff}}\right]\right]}{m^{2}},
\end{aligned}
$$

where $\lambda_{\|}$and $\lambda_{\perp}$ are dimensionless longitudinal and transverse damping parameters given by

$$
\lambda_{\|}=\lambda \frac{2 T}{3 T_{c}^{\mathrm{MF}}}, \quad \lambda_{\perp}=\lambda\left(1-\frac{T}{3 T_{c}^{\mathrm{MF}}}\right)
$$

for $T<T_{c}^{\mathrm{MF}}$ and the same with $\lambda_{\perp} \Rightarrow \lambda_{\|}$for $T>T_{c}^{\mathrm{MF}}$, where $T_{c}^{\mathrm{MF}}$ is the mean-field Curie temperature. Obviously, the damping parameters $\lambda_{\|}$and $\lambda_{\perp}$ depend on the Langevin field. The correlation between the spectrum of the Langevin field and the damping parameters has been recently investigated by (Atxitia et al., 2009).

The LLB equation (10) was shown to capture the complex physics revealed by the atomistic model, in particular, the variation in the magnetization magnitude during reversal and the increase in damping with temperature (Chubykalo-Fesenko et al., 2006). The longitudinal and transverse relaxation times calculated from the LLB equation also agree well with those calculated from the atomistic model. The LLB equation could therefore serve in the future as a basis for an improved micromagnetics at elevated temperatures. Particularly interesting would be its application in the area of multiscale simulations where atomistic simulations of nanometer-size areas are linked to micromagnetic regions to extend those calculations to macroscopic length scales.

\section{Interaction of photons and spins}

We now discuss the possibility of a direct interaction between photons and spins using energy considerations (Pershan, 1963). It can be shown that the thermodynamical potential $\Phi$ of an isotropic, nonabsorbing, magnetically ordered medium with static magnetization $\mathbf{M}(0)$ in a monochromatic light field $\mathbf{E}(\omega)$ includes a term

$$
\Phi=\alpha_{i j k} E_{i}(\omega) E_{j}(\omega)^{*} M_{k}(0),
$$

where $\alpha_{i j k}$ is the magneto-optical susceptibility (Pitaevskii, 1961; van der Ziel et al., 1965; Pershan et al., 1966; Landau and Lifshitz, 1984; Kabychenkov, 1991). The existence of this potential arises from the absence of dissipation; moreover, in the case of an isothermal process considered here, this is equivalent to the time-averaged free energy of the system; see (Pershan, 1963; Nye, 2004) for details.

In the electric dipole approximation the linear optical response of a medium to a field $\mathbf{E}(\omega)$ is defined by the optical polarization $\mathbf{P}(\omega)=\partial \Phi / \partial \mathbf{E}(\omega)^{*}$. From Eq. (12) one can easily see that the optical polarization $\mathbf{P}(\omega)$ should have a contribution $\mathbf{P}^{(m)}$ proportional to the magnetization $\mathbf{M}$, 


$$
P_{i}^{(m)}(\omega)=\alpha_{i j k} E_{j}(\omega) M_{k}(0) .
$$

From this equation one can determine that, when linearly polarized light is transmitted through a magnetized medium, the polarization plane of the light gradually rotates over an angle $\theta_{F}$ given by

$$
\theta_{F}=\frac{\alpha_{i j k} M_{k}(0) \omega L}{c n},
$$

where $c$ is the speed of light in vacuum, $n$ is the refraction coefficient of the medium, and $L$ is the propagation distance of the light in the medium (Zvezdin and Kotov, 1997). Equation (14) describes the so-called magnetooptical Faraday effect discovered by M. Faraday in 1846 (Faraday, 1846). This polarization rotation is the result of spin-orbit interaction which creates an asymmetry of the electronic wave functions, leading to a rotation of the excited dipolar currents (Argyres, 1955).

The magneto-optical Faraday effect demonstrates that a magnetically ordered medium can indeed affect photons and change the polarization of light (Zvezdin and Kotov, 1997). Is the inverse phenomenon feasible, that is, is it possible that polarized photons affect the magnetization? From Eq. (12) one can find that an electric field of light at frequency $\omega$ will act on the magnetization as an effective magnetic field $\mathbf{H}^{\text {eff }}$ directed along the wave vector of the light $\mathbf{k}$ :

$$
H_{k}=-\frac{\partial \Phi}{\partial M_{k}}=\alpha_{i j k} E_{i}(\omega) E_{j}(\omega)^{*} .
$$

In isotropic media, $\alpha_{i j k}$ is a fully antisymmetric tensor with a single independent element $\alpha$. Therefore, Eq. (15) can be rewritten as

$$
\mathbf{H}=\alpha\left[\mathbf{E}(\omega) \times \mathbf{E}(\omega)^{*}\right] .
$$

From this it becomes obvious that right- and left-handed circularly polarized waves should act as magnetic fields of opposite sign (Pitaevskii, 1961; van der Ziel et al., 1965; Pershan et al., 1966; Landau and Lifshitz, 1984). Therefore, it is seen from Eq. (16) that, in addition to the well-known magneto-optical Faraday effect where the polarization of light is affected by the magnetization $\mathbf{M}$, the same susceptibility $\alpha$ also determines the inverse optomagnetic phenomenon: circularly polarized light affects the magnetization via the inverse Faraday effect. Thus in a thermodynamical approach, the effect of light on spins in a magnetically ordered material can be described by the Landau-Lifshitz equation, where the magnetic field is generated by light via the inverse Faraday effect.

We note, however, that Eq. (16) has been derived for a monochromatic optical excitation in the approximation of thermal equilibrium. It is therefore interesting to consider the situation when the product $\mathbf{E}(\omega) \times \mathbf{E}(\omega) *$ changes much faster than the fundamental time scales in a magnetically ordered material, given by the spin precession period and the spin-lattice relaxation time (Pershan et al., 1966). Consider the excitation of spins by a laser pulse with duration $\Delta t=100$ fs. Using Fourier trans-

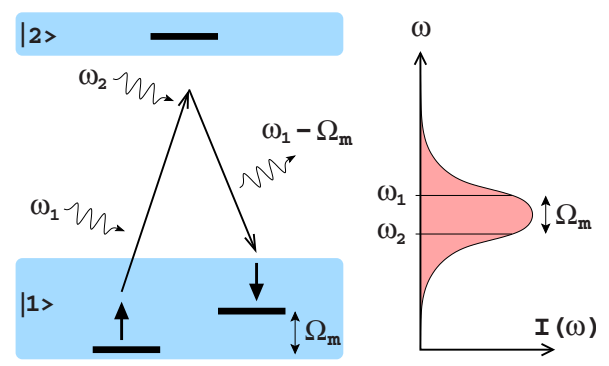

FIG. 2. (Color online) Illustration of stimulated coherent Raman scattering, a possible microscopic mechanism responsible for an ultrafast optically generated magnetic field. Two frequency components of the electromagnetic radiation from the spectrally broad laser pulse take part in the process. The frequency $\omega_{1}$ causes a transition into a virtual state with strong spin-orbit coupling. Radiation at the frequency $\omega_{2}$ stimulates the relaxation back to the ground state together with the creation of a magnon.

formation, one can see that such a laser pulse, being ultrashort in the time domain, is spectrally broad $(\Delta \omega$ $\sim 5 \mathrm{THz}$ ). Such laser excitation of spins is conveniently described in the frequency domain (see Fig. 2). Initially the electron is in the ground state $|1\rangle$ and its spin is up. If the state is nondegenerate, being an orbital singlet, the spin-orbit coupling for the electron in this state can be neglected. If we act on this electron with a photon, during the optical transition the wave function of the electron becomes a superposition of several eigenstates. This will effectively increase the orbital momentum of the electron, leading to an increase in the interaction between spins and orbital moments and thus resulting in an increase in the probability of a spin-flip process. If the energy of the photon is smaller than the gap between the ground state $|1\rangle$ and the nearest excited state $|2\rangle$, the photon will not excite any real electronic transition but just result in a spin flip of the electron in the ground state. In other words, the spin flip in the ground state is due to the fact that circularly polarized light mixes a fraction of the excited-state wave function into the ground state (Pershan et al., 1966). This process will be accompanied by the coherent reemission of a photon of energy $\hbar \omega_{2}=\hbar\left(\omega_{1}-\Omega_{m}\right)$. In magnetically ordered materials, $\hbar \Omega_{m}$ corresponds to the energy of a magnon. Moreover, such a laser-induced spin-flip process can be coherently stimulated if both frequencies $\omega_{1}$ and $\omega_{2}$ are present in the laser pulse (see Fig. 2). The time of the spin-flip process $\tau_{\mathrm{sf}}$ is given by the energy of the spinorbit interaction in the perturbed ground state $E_{\mathrm{SO}}$. For materials with a large magneto-optical susceptibility, the energy of the spin-orbit coupling may exceed $50 \mathrm{meV}$ (Kahn et al., 1969), and thus the spin-flip process can be as fast as $\tau_{\mathrm{sf}} \sim \hbar / E_{\mathrm{SO}} \sim 20 \mathrm{fs}$. This microscopic picture shows that the generation of effective magnetic fields by circularly polarized light should also work on the subpicosecond time scale.

Note that the spin-flip process as described above is allowed in the electric dipole approximation (Shen and Bloembergen, 1966). This mechanism is much more ef- 
fective than magnetic dipole transitions and does not require annihilation of a photon. It means that the energy transfer from photons to spins (magnons) is realized via an inelastic scattering process. While some photons lose a small part of their energy, the total number of photons remains unchanged. It is important to note that in a spin flip via stimulated Raman scattering, as described above, the stimulating and reemitted photons have identical polarization, implying that such a photoninduced spin flip is not accompanied by a loss of the angular momentum of the photons. It has been argued, on the other hand, that even in the absence of spinlattice interaction, the angular momentum can be supplied via the change in the direction of the pump light, i.e., via the linear momentum of photons (Woodford, 2009). This, however, still needs to be confirmed in an experiment.

\section{EXPERIMENTAL TECHNIQUES}

\section{A. Pump-and-probe method}

The investigation of ultrafast spin dynamics triggered by a picosecond or subpicosecond laser pulse requires methods that allow detection of the changes in the magnetization in a medium with subpicosecond temporal resolution. Practically all the studies of ultrafast laserinduced magnetization dynamics employ some kind of pump-and-probe method, where the magnetization dynamics is triggered by a short laser pulse in the optical range (pump), while detection (probe) of the laserinduced magnetic changes is done with the help of a second pulse of electromagnetic radiation (Shah, 1996). Analysis of the properties of the probe pulse after interaction with the medium for different time separations between pump-and-probe pulses allows reconstruction of the ultrafast laser-induced magnetic changes actually taking place in the medium. The duration of the probe pulse defines the temporal resolution, while the spectrum of the probe is crucial for the sensitivity of such measurements. Choosing the probe pulse in the farinfrared, optical, ultraviolet, or x-ray spectral ranges, one defines the electronic transitions responsible for the interaction of the probe pulse with the medium. As a result, all these spectroscopic methods are characterized by different sensitivity to spin and orbital degrees of freedom. Therefore measurements in the far-infrared, optical, ultraviolet, or x-ray spectral ranges allow one to obtain four different views on the same phenomenon and thus obtain the most complete information.

\section{B. Optical probe}

The interaction of a medium with a probe pulse in the visible range or its vicinity $(0.4-10 \mu \mathrm{m})$ can be described in the electric dipole approximation. Using energy considerations, one can analyze such an interaction by considering the thermodynamical potential $\Phi$. For an isotropic nondissipating, magnetically ordered medium with static magnetization $\mathbf{M}(0)$ or antiferromagnetic vector
$\mathbf{I}(0)$ in a monochromatic light field $\mathbf{E}(\omega)$, neglecting terms of order higher than 3 in $\mathbf{E}(\omega)$, $\Phi$ can be written as

$$
\begin{aligned}
\Phi= & \chi_{i j}^{(l)} E_{i}(\omega)^{*} E_{j}(\omega)+\alpha_{i j k}^{(l)} E_{i}(\omega)^{*} E_{j}(\omega) M(0)_{k} \\
& +\beta_{i j k}^{(l)} E_{i}(\omega)^{*} E_{j}(\omega) l(0)_{k}+\chi_{i j k}^{(n l)} E_{i}(2 \omega)^{*} E_{j}(\omega) E_{k}(\omega) \\
& +\alpha_{i j k l}^{(n l)} E_{i}(2 \omega)^{*} E_{j}(\omega) E_{k}(\omega) M(0)_{l} \\
& +\beta_{i j k l}^{(n l)} E_{i}(2 \omega)^{*} E_{j}(\omega) E_{k}(\omega) l(0)_{l},
\end{aligned}
$$

where $\chi_{i j}^{(l)}, \alpha_{i j k}^{(l)}, \beta_{i j k}^{(l)}, \chi_{i j k}^{(n l)}, \alpha_{i j k l}^{(n l)}$, and $\beta_{i j k l}^{(n l)}$ are tensors that define the optical properties of the medium, and the superscripts $l(n l)$ indicate the linear (nonlinear) response, respectively (Pershan, 1963). Equation (17) is a generalization of Eq. (12) from which the Faraday effect was derived.

Similarly, because of the polarization $\mathbf{P}^{(m)}$, one can obtain polarization rotation of light upon reflection from a magnetized medium; this phenomenon is called the magneto-optical Kerr effect (MOKE). Therefore, linear magneto-optical effects can be used as a probe of the magnetization of a medium and, using modulation techniques, can be sensitive to even (sub)monolayer magnetic films (Qiu and Bader, 1999).

Considering in Eq. (17) the terms linear in the antiferromagnetic vector $\mathbf{l}(0)$, it is easy to see that linear optics can also serve as a probe of magnetic order in geometries where no Faraday or Kerr effect is present or in materials with no net magnetization, such as antiferromagnets or ferrimagnets at their compensation point (Chaudhari et al., 1973). It is important to note that all linear magneto-optical phenomena are sensitive to certain projections of the magnetic vectors ( $\mathbf{M}$ and $\mathbf{I})$ and can be observed in all media, irrespective of their crystal symmetry or crystallographic orientation (Smolenskil et al., 1975; Ferré and Gehring, 1984).

Although the magneto-optical Faraday effect (i.e., magnetic circular birefringence) and the magnetic linear birefringence at a frequency $\omega$ do not require absorption at this frequency, causality does not allow complete neglect of absorption. Because of the Kramers-Kronig relations, an observation of circular or linear birefringence in a certain spectral range should be accompanied by similar effects of polarization-dependent absorption in another spectral domain. Such effects are known as magnetic circular dichroism and magnetic linear dichroism and are often employed as efficient probes of magnetization as well (Zvezdin and Kotov, 1997; Kottler et al., 1998).

In the nonlinear optical approximation of Eq. (17), one should take into account the terms of third order with respect to the electric field of light. In that approximation, the optical field $\mathbf{E}(\omega)$ is able to induce a polarization in the medium at the double frequency $\mathbf{P}(2 \omega)$. The appearance of this polarization results in the phenomenon of second-harmonic generation when the medium excited by the optical field $\mathbf{E}(\omega)$ generates light at the double frequency $2 \omega$. The intensity of the secondharmonic light in ferromagnets or antiferromagnets can be found to be 


$$
\begin{aligned}
& I(2 \omega) \sim\left|P(2 \omega)_{i}\right|^{2} \\
&= {\left[\chi_{i j k}^{(n l)} E_{j}(\omega) E_{k}(\omega)+\alpha_{i j k l}^{(n l)} E_{j}(\omega) E_{k}(\omega) M(0)_{l}\right]^{2} } \\
& \text { or } \quad\left[\chi_{i j k}^{(n l)} E_{j}(\omega) E_{k}(\omega)+\alpha_{i j k l}^{(n l)} E_{j}(\omega) E_{k}(\omega) l(0)_{l}\right]^{2},
\end{aligned}
$$

respectively.

From this one can conclude that the second-harmonic generation can also be a measure of the magnetization $\mathbf{M}(0)$ or antiferromagnetic vector $\mathbf{I}(0)$ in the medium. However, in contrast to linear magneto-optical effects, the second harmonic in the electric dipole approximation is generated only in media in which the crystallographic symmetry does not contain a center of inversion. In centrosymmetric media the second-harmonic generation is thus only allowed at surfaces and interfaces where the bulk symmetry is broken. Consequently, the magnetic second-harmonic generation (MSHG) technique can serve as a unique tool for probing surface and interface magnetism (Kirilyuk and Rasing, 2005).

Since the optical response of media in the visible spectral range is dominated by electric dipole transitions and the selection rules for such transitions do not allow a spin flip, the sensitivity of light to magnetic order in case of both linear and nonlinear magneto-optical effects requires a strong spin-orbit interaction. A strong coupling between spins and orbitals results in substantial values of the $\alpha$ and $\beta$ components in Eq. (17) so that one can use the magneto-optical effects to obtain information about the magnetic state of a medium.

Interpretation of the results of magneto-optical studies of solids is hampered by the fact that optical transitions in the visible spectral range are relatively broad. The corresponding spectral lines often overlap even for dielectric materials (Lever, 1984). For an analysis of optical excitations of metals, one has therefore to consider a continuum of transitions, making a theoretical description of the magneto-optical spectra an extremely difficult problem. Moreover, to apply them for ultrafast studies one has to take into account a possible nonequilibrium behavior of the system that also strongly affects the magneto-optical response (Oppeneer and Liebsch, 2004).

In addition, magneto-optical effects are only proportional to $\mathbf{M}$ and $\mathbf{I}$ and are thus only indirect probes of spin ordering. This results in a number of uncertainties in the interpretation of time-resolved magneto-optical measurements. Laser excitation can change populations of the excited states (this process is called "bleaching") and modify the symmetry of the ground state, and thus may result in changes in the magneto-optical response of a medium even if its magnetic order and magnetization vector have not been affected (Koopmans et al., 2000; Regensburger et al., 2000; Oppeneer and Liebsch, 2004). In terms of a thermodynamical description of the magneto-optical effects, such a situation corresponds to laser-induced changes in the tensors $\alpha_{i j k}^{(l)}, \beta_{i j k}^{(l)}, \alpha_{i j k l}^{(n l)}$, and $\beta_{i j k l}^{(n l)}$. However, we stress here that, although a thermodynamical description is suitable to demonstrate the fact that optical phenomena can serve as a probe of magnetic order, an explanation of femtosecond laser-induced ef- fects in magnets in terms of time-dependent tensors cannot be expected to fully describe the phenomena that actually take place at the ultrafast time scale. Laserinduced magneto-optical Kerr or Faraday effects in pump-probe experiments should be described in terms of a nonlinear polarization of third order, as done by Sham (1999) and Kimel et al. (2001). Such a theory can be easily developed for a simple two- or three-level system. However, a proper theoretical framework that allows an adequate description of the time-resolved pump-probe magneto-optical experiments in metals, magnetic semiconductors, and even dielectrics remains challenging, and the number of approaches is limited (Oppeneer and Liebsch, 2004; Vernes and Weinberger, 2005; Zhang et al., 2009).

Recently Zhang et al. claimed the emergence of a new "paradigm of the time-resolved magneto-optical Kerr effect for femtosecond magnetism" (Zhang et al., 2009). Despite the timeliness and relevance of this result, it should be noted that this article (a) does not consider pump-and-probe experiments at all since there is only one pulse in the model; (b) considers only the $M_{x y}$ component of the magnetization tensor, while the relation between the calculated off-diagonal components of the magnetization tensor $M_{x y}$ and the net magnetization of the sample $M_{z z}$ remains unclear. The work of Zhang et $a l$. is no doubt a step toward a better understanding of the results of time-resolved magneto-optical experiments on magnetic metals. However, as the study involves quite some simplifications and leaves many questions unanswered, a new paradigm might be a slight overstatement.

\section{Ultraviolet probe and spin-polarized electrons}

The excitation of a medium with photons with an energy larger than the work function, the difference between the Fermi level and the vacuum energy, may result in an emission of electrons from the surface. Photoelectron spectroscopies facilitate access to the static electronic structure of condensed matter as it enables one to obtain information about both the energy and momentum distribution of the electrons (Hüfner, 1995). With spin-sensitive detectors one can detect the average spin polarization of the emitted electrons, providing thus direct access to the spin distribution of the electron gas in a metal. This is in contrast to the magneto-optical methods, which are indirect. For this reason the interpretation of time-resolved photoemission experiments is much more straightforward than that of time-resolved magneto-optical measurements (Vaterlaus, Beutler, and Meier, 1990, 1991; Scholl et al., 1997; Lisowski et al., 2005). The disadvantages of photoemission are the strong requirements on the conductivity and the quality of the surface. Consequently, the technique can be applied to a limited set of compounds, mainly, metals or half metals.

In principle, important insights into the dynamics and relaxation processes of excited electrons can be obtained with the help of two-photon photoemission (2PPE) that 
is also spin, energy, and time resolved (Aeschlimann et al., 1997). This is a two-step process, where an ultrashort pump laser pulse excites electrons from the Fermi level, and the transient population of the intermediate excitation is probed by a second pulse which promotes the still excited electrons to the vacuum where their energy is analyzed.

The nonlinearity of the two-photon process leads to an increase in the 2PPE yield when the pulses are spatially and temporarily superimposed. As long as the two laser pulses temporarily overlap it is obvious that an electron can be emitted by absorbing just one photon from each pulse. However, if the pulses are temporarily separated, then an excited electron from the first pulse is able to absorb a photon from the second pulse only as long as the inelastic lifetime of the intermediate state exceeds the delay or if the normally unoccupied electronic state is refilled by a secondary electron. Because of the precise measurement of the time delay between the two pulses, this technique allows analysis of relaxation times which are considerably shorter than the laser pulse duration; see, e.g., Schmidt et al. (2005).

\section{Far-infrared probe}

The energy of far-infrared photons falls in the range where metals have an intense response of their free electrons. This Drude response of free electrons to electric field transients allows one to obtain information about the conductivity of the material. Therefore, timeresolved far-infrared spectroscopy has become a powerful tool for the investigation of phase transitions in strongly correlated systems, such as superconductorconductor and metal-dielectric transitions (Averitt et al., 2001). If the conductance of the free-electron gas is sensitive to the magnetic order, it can be used as an indirect probe of magnetic phase transitions as well. It is interesting to note that the frequencies of magnetic resonance of most antiferromagnets lie in the range from $100 \mathrm{GHz}$ to $3 \mathrm{THz}$. Moreover, several rare-earth ions have spin-flip transitions in the range between $100 \mathrm{GHz}$ and $10 \mathrm{THz}$. Thus terahertz spectroscopy can potentially deliver a direct access to the spin system and thus be a unique probe of transient laser-induced magnetic changes complementary to optical or ultraviolet methods (Hilton et al., 2006).

\section{E. X-ray probe}

The development of synchrotron x-ray sources has enabled the generation of linearly and circularly polarized radiation with intensities sufficient for the measurements of magnetic circular and magnetic linear dichroism in the X-ray domain (XMCD and XMLD, respectively) (van der Laan et al., 1986; Schütz et al., 1987; Schneider et al., 1993; Stöhr et al., 1993). Similar to magneto-optical phenomena in the visible spectral range, XMCD and XMLD can be employed in order to deduce the orientation of the magnetization and antiferromagnetic vector, respectively. However, in contrast to the optical spectral range, in the range of x-rays magnetic materials are characterized by relatively narrow transitions at well-defined energies, so that x-ray absorption techniques are characterized by elemental and even chemical specificity. Moreover, because spectral lines in the $\mathrm{x}$-ray regime are narrow and well separated, one can use so-called sum rules to obtain quantitative information about spin and orbital moments of the ground state (Carra et al., 1993).

Because of the advantages of x-ray techniques, timeresolved XMCD measurement with subpicosecond temporal resolution would provide novel and unique insights into ultrafast laser-induced magnetic changes. The development of such a method has become one main goal in the physics of magnetism over the last ten years. The first XMCD results with 100 fs temporal resolution have recently been reported (Stamm et al., 2007). It should be noted, however, that ultrafast measurements in the $\mathrm{x}$-ray spectral range are still technically challenging, requiring much effort. Also, theory for the interpretation of ultrafast XMCD measurements still needs to be developed (Carva et al., 2009).

\section{THERMAL EFFECTS OF LASER EXCITATION}

\section{A. Ultrafast demagnetization of metallic ferromagnets}

Metallic ferromagnets happen to be complex systems to understand because of the itinerant character of their magnetism. At the same time, metallic magnets are used in numerous applications, from power transformers and sensors to data storage and spintronics. It is therefore not surprising that the experimental studies of subpicosecond magnetization dynamics has started with such "simple" systems as Fe (Kampfrath et al., 2002; Carpene et al., 2008), $\mathrm{Ni}$ (Beaurepaire et al., 1996), or Gd samples. Despite some progress, the results are still being debated, both theoretically and experimentally.

The first ultrafast time-resolved studies of the impact of laser pulses on the magnetization were done on $\mathrm{Ni}$ and $\mathrm{Fe}$ using picosecond laser pulses. However, these were not successful in observing any magnetic effects up to the melting point of the samples (Agranat et al., 1984; Vaterlaus, Beutler, and Meier, 1990). Later, using timeresolved spin-polarized photoemission as a probe of the magnetization Vaterlaus et al. succeeded in estimating the spin-lattice relaxation time in $\mathrm{Gd}$ films to be $100 \pm 80$ ps (Vaterlaus, Beutler, and Meier, 1991). The difficulties in the measurements were related to the fact that the available laser pulses were of the same duration or even longer ( $60 \mathrm{ps}$ to $10 \mathrm{~ns}$ ) than the relevant time scales. Only later was it realized that, with such long excitations, the various parts of the system were always in equilibrium with each other so that the system followed the excitation profile. To study the intrinsic time scales mentioned earlier, much shorter stimuli are required. 


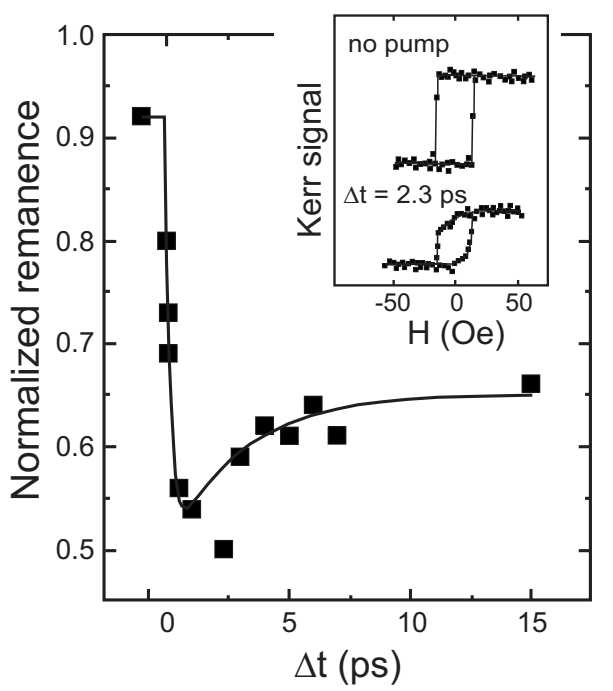

FIG. 3. Remanent MO contrast measured for a nickel thin film as a function of time after exciting by a 60 fs laser pulse. The results demonstrate ultrafast loss of the magnetic order of the ferromagnetic material within a picosecond after laser excitation. From Beaurepaire et al., 1996.

\section{Experimental observation of ultrafast demagnetization}

Beaurepaire et al. were the first to use $60 \mathrm{fs}$ laser pulses to measure both the transient transmittivity and the linear MOKE of $22 \mathrm{~nm}$ Ni films (Beaurepaire et al., 1996), see Fig. 3. It was estimated from the transient reflectivity that the electron thermalization time is about $260 \mathrm{fs}$ and the electron temperature decay constant is 1 ps. In contrast, the spin temperature deduced from the time dependence of hysteresis loops reached its maximum around 2 ps only. Thus, different electron and spin dynamics were postulated. On the other hand, in the following MSHG experiments by Hohlfeld et al., a similar electron thermalization time was obtained ( $280 \mathrm{fs})$, but no delay between electron excitation and the loss of magnetization was seen (Hohlfeld et al., 1997). In both cases, the observed dynamics was much faster than what could be expected on the basis of the spin-lattice relaxation time. In fact, ignoring a possible small difference between electron and spin temperature, these results showed that the excitation by an ultrashort laser pulse leads to ultrafast heating of the electronic system. As a result, the magnetization will decrease equally fast following its normal temperature dependence $M=M\left(T_{e}\right)$, where $T_{e}$ is the electron temperature. In the two-photon photoemission of Scholl et al., two different demagnetization processes were observed, a fast one in less than $300 \mathrm{fs}$, and a very slow one around $500 \mathrm{ps}$ which was ascribed to the excitation of spin waves (Scholl et al., 1997). Femtosecond demagnetization was also demonstrated by Stamm et al. using XMCD (Stamm et al., 2007).

While it has been generally accepted that the magnetization follows the electron temperature with a possible delay between the electron excitation and the magnetic breakdown of no more than $50 \mathrm{fs}$, questions arose about

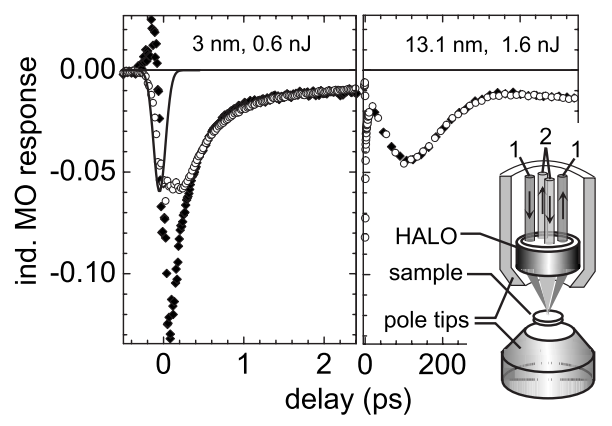

FIG. 4. Comparison of induced MO ellipticity (open circles) and rotation (filled diamonds) for a $\mathrm{Cu}(111) / \mathrm{Ni} / \mathrm{Cu}$ epitaxial film and Ni thickness and pulse energy as indicated. The inset depicts the polar configuration with pump (1) and probe (2) beams. From Koopmans et al., 2000.

the interpretation of the experimental results. For example, is the magneto-optical response from a nonequilibrium system indeed proportional to the magnetization? A study on $\mathrm{Cu} / \mathrm{Ni} / \mathrm{Cu}$ wedges (Koopmans et al., 2000) demonstrated that during the first hundreds of femtoseconds the dynamical evolution of the Kerr ellipticity and rotation do not coincide (see Fig. 4), breaking down the proportionality between the magnetization and the Voigt vector that is the basis of magneto-optics. Such breakdown is indeed a consequence of the out-ofequilibrium character of the electron system immediately after the femtosecond excitation. Only after the electronic equilibration process can one reliably deduce the change in the magnetization from changes in Faraday or Kerr rotation. Such arguments were used to cast doubt on ultrafast (instantaneous) light-induced changes in the magnetization (Koopmans et al., 2000).

Nevertheless, using laser pulses as short as 20 fs duration and carefully separating the dynamics of the diagonal and the nondiagonal elements of the time-dependent dielectric tensor, it has been shown that a significant demagnetization can be obtained at a sub-100 fs time scale, for example, in $\mathrm{CoPt}_{3}$ (Guidoni et al., 2002) (see Fig. 5). Moreover, it has been shown by time-resolved photoemission that the exchange splitting between majority and minority spin bands is affected at a similar time scale (Rhie et al., 2003).

The breakdown between the magneto-optical response and magnetization in ultrafast pump-probe experiments has been also supported by ab initio calculation of the magneto-optical Kerr effect in $\mathrm{Ni}$ (Oppeneer and Liebsch, 2004). By evaluating the complex conductivity tensor of $\mathrm{Ni}$ for nonequilibrium electron distributions, Oppeneer and Liebsch considered dichroic bleaching and state-blocking effects. It was shown that the conductivity tensor and therefore the complex Kerr angle can be substantially modified so that the Kerr rotation and ellipticity are no longer proportional to the magnetization of the sample and the Kerr response at ultrashort times can therefore not be taken as a measure of demagnetization. For $\mathrm{CoPt}_{3}$, the role of dichroic bleaching and state-blocking effects in ultrafast magneto-optical pump-probe experiments has been veri- 

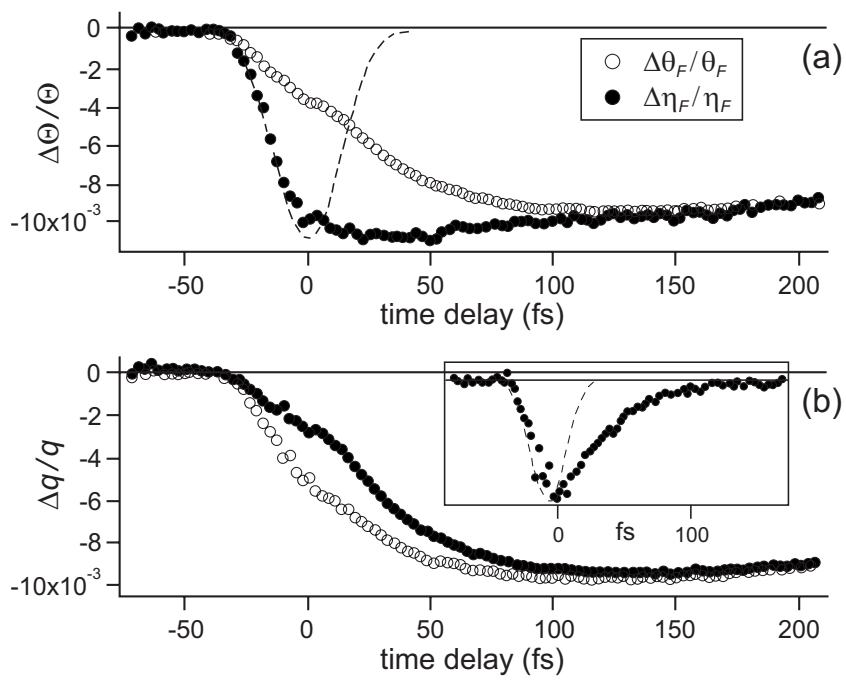

FIG. 5. Ultrafast laser-induced dynamics in $\mathrm{CoPt}_{3}$. (a) Timeresolved Faraday MO signals, rotation (open circles), and ellipticity (filled circles). The pump-probe cross correlation (dashed line) is displayed for reference. (b) Short-delay relative variations of real and imaginary dielectric tensor components retrieved from the data in (a). For $t \leqslant 100 \mathrm{fs}$, the real and imaginary parts follow different dynamics. From Guidoni et al., 2002.

fied experimentally, employing measurements where the wavelength of the probe was varied in the broad spectral range between 500 and $700 \mathrm{~nm}$ (Bigot et al., 2004). If bleaching and state-blocking effects are indeed present, one would expect to observe a spectral dependence in the magneto-optical response, associated with the population bleaching. However, the spectral response was found to be rather flat, and it was concluded that the magneto-optical signal predominantly reflects the spin dynamics in this ferromagnet. We mention that relatively small magneto-optical signals from $\mathrm{Ni}$ did not allow the performance of similar measurements in this metal. Therefore, strictly speaking the conclusions of Bigot et al. (2004) cannot be expanded to other metals. Moreover, a saturation of ultrafast laser-induced demagnetization at high excitation densities has recently been observed in $\mathrm{Ni}$ and has been explained in terms of band filling effects (Cheskis et al., 2005).

Another signature of ultrafast laser-induced demagnetization is emission in the terahertz spectral range. If the magnetization of a ferromagnetic film is changed on a subpicosecond time scale, an electromagnetic wave will be generated according to Maxwell's equations. This radiated wave thus contains information about the intrinsic spin dynamics. Similar ideas were used to investigate carrier dynamics in semiconductors and Cooper-pair breaking in superconductors. Beaurepaire et al. were first to apply this technique on thin Ni films in order to obtain an extra proof of the subpicosecond demagnetization (Beaurepaire et al., 2004). A rather standard electro-optic sampling technique in a 0.5 -mm-thick ZnTe crystal was used [for a review see Ferguson and Zhang (2002)].

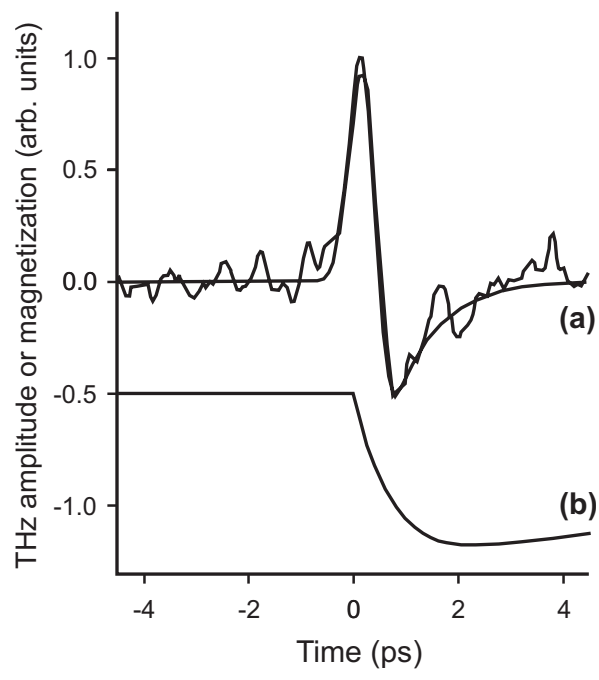

FIG. 6. Laser-induced generation of $\mathrm{TH}_{2}$ radiation. (a) The $\mathrm{THz}$ pulse generated upon ultrafast laser heating of 42 - $\AA$-thick in-plane magnetized $\mathrm{Ni}$ film. The smooth line is a simulation assuming the time-dependent magnetization of (b) convoluted with a Gaussian instrument response function (full width at half maximum 540 fs). From Beaurepaire et al., 2004.

The electric field thus measured upon ultrafast laserinduced demagnetization of the thin $\mathrm{Ni}$ film is shown in Fig. 6(a). If one assumes that pump pulses coherently excite the film, producing a time-varying magnetization, the electric field in the far field (polarized orthogonal to the magnetization vector $M_{x}$ ), propagating in the $z$ direction is

$$
E_{y}(t)=\frac{\mu_{0}}{4 \pi^{2} r} \frac{\partial^{2} M_{x}}{\partial t^{2}}(t-r / c)
$$

where $r$ is the distance to the dipole. Thus the measured transient electric field emitted by the sample is given by the second derivative of the magnetization.

With this in mind, and assuming the magnetization variation as shown in Fig. 6(b), the solid curve of Fig. 6(a) is obtained, showing a perfect correspondence with the experimentally observed terahertz radiation. It has also been verified that the outgoing terahertz wave is indeed polarized along the $y$ axis and that the effect is independent of the incoming polarization of the visible pump light.

Similar experiments have also been performed on thin Fe films (Hilton et al., 2004). There the terahertz emission was found to have two contributions. One part of the terahertz signal depended on the polarization of the pump beam and was attributed to the nonlinear effect of optical rectification. Another part did not depend on the pump polarization and likely originated from the ultrafast demagnetization.

Finally, we mention that ultrafast laser-induced demagnetization of metals can be accompanied by a change in magnetization reversal behavior (Weber et al., 2004; Wilks et al., 2005; Roth et al., 2008; Xu et al., 2008). 


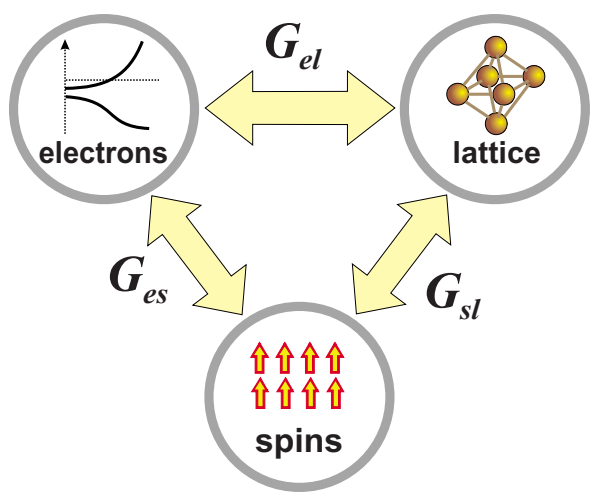

FIG. 7. (Color online) Interacting reservoirs (carriers, spins, and lattice) in the three-temperature model as suggested by Agranat et al. (1984). Some of the possible interaction channels, such as spin lattice and electron-phonon interactions, are considered to be well understood. The direct electron-spin coupling is a subject of current research and active debates.

\section{Phenomenological three-temperature model}

Already, since the first laser-induced magnetization dynamics experiments, it has become clear that the resulting demagnetization is a complicated process, where various components of the system are participating that involve different relaxation processes.

The interactions in such a system can be qualitatively described with a help of a single model that contains three separate but interacting reservoirs, namely, the electrons, lattice, and spins (see Fig. 7). These three reservoirs are connected by interactions of different origin and efficiency. A particular effective temperature can be assigned to each of these reservoirs. Note, however, that this assignment is possible only if a certain equilibrium is assumed within the considered subsystem. Given the short time scales, this assumption is not always valid, imposing a limit on the validity of this model.

The three-temperature model describes the temporal evolution of the system by three coupled differential equations,

$$
\begin{aligned}
& C_{e} d\left(T_{e}\right) / d t=-G_{e l}\left(T_{e}-T_{l}\right)-G_{e s}\left(T_{e}-T_{s}\right)+P(t), \\
& C_{s} d\left(T_{s}\right) / d t=-G_{e s}\left(T_{s}-T_{e}\right)-G_{s l}\left(T_{s}-T_{l}\right), \\
& C_{l} d\left(T_{l}\right) / d t=-G_{e l}\left(T_{l}-T_{e}\right)-G_{s l}\left(T_{l}-T_{s}\right),
\end{aligned}
$$

where $G_{i j}$ represents the coupling between the $i$ th and $j$ th baths, $C_{i}$ describes their heat capacity, $T_{i}$ is the temperature of the corresponding system, and $P(t)$ is the optical input. The $G_{i j}$ coefficients are phenomenological parameters that once determined tell us how strong a particular link is but at the same time tell us nothing about the nature of the interaction. Depending on the heat capacities $C_{j}$, effective temperature differences can be very large. For example, since the electron heat capacity is typically one to two orders of magnitude smaller than that of the lattice, $T_{e}$ may reach several thousand Kelvin within the first tens of femtoseconds after excitation, while the lattice remains relatively cold

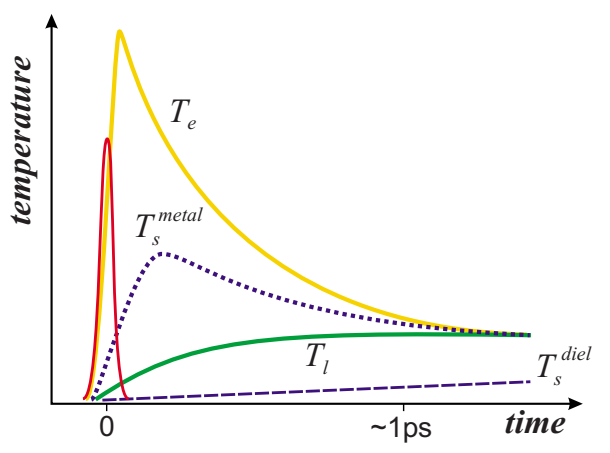

FIG. 8. (Color online) Temporal behavior of electron, lattice, and spin temperatures following excitation with a short laser pulse. The behavior of the spin temperature is shown for both strong coupling (metals) and weak coupling (dielectrics) cases.

(Fatti et al., 2000) even after the equilibration process. Schematically the process of the equilibration between different reservoirs following the excitation with a femtosecond laser pulse is shown in Fig. 8.

A typical scenario of the processes leading to a laserinduced demagnetization is the following. At optical frequencies, only electrons are able to respond to an electromagnetic excitation, absorbing or scattering the photons practically instantaneously. Thus, in the first step (i) the laser beam hits the sample and creates electron-hole pairs (hot electrons) on a time scale of $\sim 1$ fs; (ii) the electronic system equilibrates at elevated temperatures $T_{e}$ by electron-electron interactions within $50-500 \mathrm{fs}$, depending on the system; and (iii) the equilibrated electronic excitations decay via phonon cascades on a time scale given by the electron-phonon interaction time (within $100 \mathrm{fs}-1 \mathrm{ps}$ for metals) and heat up the lattice, increasing $T_{l}$. Thus, at the end of a picosecond, electron and lattice systems are in thermal equilibrium with each other. What happens with the spins?

As the essence of magnetization is angular momentum, apart from the energy, angular momentum conservation needs to be considered in magnetic systems (Zhang and George, 2008). Also, in the process of demagnetization, a certain amount of angular momentum must be taken away from the spin system. It is therefore crucial to disclose the possible channels for this angular momentum transfer when considering the various processes involved in ultrafast demagnetization.

Generally speaking, both the electron system and the lattice should be able to absorb this angular momentum, even if only temporarily. The spin-lattice interaction is usually considered to be of the same order of magnitude as the magnetocrystalline anisotropy. The latter is rather weak $(\sim 100 \mu \mathrm{eV})$ in transition metals because of the crystal-field-induced quenching of the electron orbits. Therefore the corresponding interaction time is expected to be quite long, e.g., $300 \mathrm{ps}$ in $\mathrm{Ni}$ (Hübner and Zhang, 1998). However, after femtosecond demagnetization had been demonstrated (Beaurepaire et al., 1996), it became clear that in metals there is a much stronger coupling between the spins and the two other reservoirs. Mechanisms of an effective electron-spin or phonon- 
spin coupling responsible for a demagnetization faster than 100 fs are the subject of current research and active debates.

Recently Bigot et al. suggested that the laser-induced demagnetization of Ni could be related to a direct coupling between photons and spins (Bigot et al., 2009). In particular, a novel relativistic quantum mechanical mechanism was claimed to be responsible for the ultrafast change in magnetization. However, in this work the only direct, i.e., polarization-dependent, effect was observed during the overlap of pump and probe pulses. This is similar to earlier work by Dalla Longa et al. (2007), where an analogous effect was attributed to the effects of optical coherence. Indeed, if the pump pulse leaves the system in the same magnetic state regardless the polarization, what then is the result of that "direct coupling"? It should also be mentioned that a relativistic coupling of spins to a light field has been considered earlier for the description of magneto-optical effects in uranium compounds by Kraft et al. (1995), but their Hamiltonian is different from the one suggested by Bigot et al. In this respect, it is worth noting that it has recently been demonstrated, using polarization pulse shaping, for the case of an elementary two-level system that such instantaneous laser-induced magneto-optical effect has nothing to do with spin excitations but originates from off-resonant pumping of excited-state population, obviously present only during the action of the light pulse (Versluis et al., 2009). Clearly, on the topic of direct coupling between photons and spins, the last word has not yet been said.

\section{Spin-flip and angular momentum transfer in metals}

The interaction that is responsible for the angular momentum flow into the electron orbits is the spin-orbit coupling, about $50 \mathrm{meV}$ in ferromagnetic metals. This would correspond to a minimum relaxation time of some $20 \mathrm{fs}$, i.e., fast enough to explain the observed ultrafast effects. There are three main mechanisms through which an excited electron can flip its spin in a ferromagnetic metal. These are (i) a Stoner excitation, effective at relatively high energies (Stöhr and Siegmann, 2006); (ii) an inelastic electron-spin-wave scattering event, effective in the cases of relatively low excitation photon energy (Edwards and Hertz, 1973; Hertz and Edwards, 1973; Zhukov and Chulkov, 2009), and (iii) a single-particle-like spin-flip scattering with impurities or phonons, called the Elliott-Yafet mechanism (Elliott, 1954; Yafet, 1963). These three mechanisms are characterized by short time scales and can lead, in principle, to the observed subpicosecond changes of magnetization in metals after excitation with a laser pulse, as far as the energy relaxation is concerned. The relaxation of the energy into the lattice occurs in parallel to this and can be considered only as a factor limiting the demagnetization magnitude because of too fast energy dissipation.

In metals, the spin-flip probability is strongly affected by the band structure. The electron-electron interactions in itinerant-electron systems lead to a separation of the electronic bands for spin-up and spin-down electrons. When the Fermi level lies between these two bands, the lower-energy spin-up (or majority) states are occupied, while the higher-energy spin-down (or minority) states are not. It is this difference that leads to a net spin polarization of the conduction electrons. Moreover, bands that are exchange-split across the Fermi level also present the possibility of a unique single-particle magnetic excitation. (Such excitation is nevertheless manybody-like in the sense of a Coulomb interaction.) An electron may be removed from an occupied majority state, undergo spin reversal, and be placed in a previously unoccupied minority state. The resulting electronhole pair with opposite spins and net vector $q$ is called a Stoner excitation. Note that for each combination of $q$ and spin, a distribution of Stoner excitations is possible (Mohn, 2003).

Since Stoner excitations involve a spin reversal, these are the fundamental single-particle magnetic excitations in itinerant-electron magnets. At low energy and wave vector, the configuration of the electronic bands (the large value of exchange splitting) does not allow individual Stoner excitations. In this case, a coherent superposition of virtual excitations of wave vector $q$ can produce a collective magnetic excitation at $q$ (called spin wave) at low energy. In the regions of energymomentum space where individual Stoner excitations are (almost) allowed, the spin waves and Stoner modes will be coupled so that the former become heavily damped.

From this picture it is clear that in the low-energy region spin waves will dominate over Stoner excitations. This is true in the case of most (though not all) laserinduced magnetization dynamics experiments that use near-infrared wavelengths around $800 \mathrm{~nm}$, corresponding to a photon energy of $\sim 1.5 \mathrm{eV}$. Note, however, that no finite $q$ vector is required for on-site spin flips, while nevertheless a low-energy excitation becomes possible due to many-body effects.

In general, a possible mechanism for a flip of one single spin in a metal was studied more than 50 years ago and called Elliott-Yafet scattering (Elliott, 1954; Yafet, 1963). In this theory it is shown that a scattering event of an excited electron with a phonon changes the probability to find that electron in one of the spin states $|\uparrow\rangle$ or $|\downarrow\rangle$, thus delivering angular momentum from the electronic system to the lattice. Because of the spin-orbit coupling, an electronic state in a solid is always a mixture of the two spin states, e.g., a dominant spin-up contribution and a small spin-down contribution, defining the total crystal wave function (Steiauf and Fähnle, 2009),

$$
\psi_{\mathbf{k}, \uparrow}=a_{\mathbf{k}}(\mathbf{r})|\uparrow\rangle+b_{\mathbf{k}}(\mathbf{r})|\downarrow\rangle,
$$

where $\mathbf{k}$ is the wave vector. The potential describing the interaction of an electron with a phonon of wave vector q consists of two parts 


$$
U_{\mathbf{q}}=U_{\mathbf{q}}^{0}+U_{\mathbf{q}}^{\text {spin-orbit }}
$$

where $U_{\mathbf{q}}^{0}$ is the spin-independent part and $U_{\mathbf{q}}^{\text {spin-orbit }}$ refers to the spin-dependent part of the scattering potential. The matrix element which is of interest for demagnetization is the spin-flip matrix element between a spin-up state $\psi_{\mathbf{k}, \uparrow}$ and a spin-down state $\psi_{\mathbf{k}+\mathbf{q}, \downarrow}$ :

$$
m_{\mathbf{k}+\mathbf{q}, \downarrow ; \mathbf{k}, \uparrow}=\left\langle\psi_{\mathbf{k}+\mathbf{q}, \downarrow}\left|U_{\mathbf{q}}^{0}+U_{\mathbf{q}}^{\text {spin-orbit }}\right|_{\psi_{\mathbf{k}, \uparrow}}\right\rangle .
$$

It was the important observation of Elliott (1954) that the first part of this matrix element, originating from $U_{\mathbf{q}}^{0}$, is already nonzero because it connects the large and small components of two spinors. It was shown to be essential, however, that both parts of the potential are taken into account (Yafet, 1963).

Steiauf and Fähnle recently calculated the spin-mixing parameters in several transition metals using $a b$ initio density functional theory (Steiauf and Fähnle, 2009). The calculations showed that in $\mathrm{Co}$ and $\mathrm{Fe}$ as well as $\mathrm{Ni}$ the spin-mixing parameter is about a factor of 25 larger than the value commonly used for $\mathrm{Cu}$. Although these results support the idea that angular momentum transfer from spins to the lattice via the Elliott-Yafet-like mechanism may play an important role in the femtosecond demagnetization, one should remember that this is a single-electron model, where exchange interaction is not taken into account and hence the spin-flip events are assumed to be independent. Therefore a straightforward application of these conclusions to magnetically ordered materials would result in an oversimplified model which neglects fundamental features of magnetism.

An attempt to predict the time scale of ultrafast laserinduced demagnetization of metals has been recently performed under the assumption that the speed of the demagnetization is defined by the speed of spin-flip processes via the Elliot-Yafet mechanism (Koopmans, Kicken, et al., 2005; Koopmans, Ruigrok, et al., 2005). Using the Landau-Lifshitz-Gilbert equation (see Sec. II.A), an analytical expression was derived that connects the demagnetization time constant with the Gilbert damping parameter $\alpha$ via the Curie temperature. Basically this was an attempt to connect the characteristic times of magnon scattering at the center and at the edge of the Brillouin zone. However, in strong contrast to $a b$ initio calculations Gómez-Abal et al. (2004) unavoidably neglected the electronic band structure of the material in light-matter interactions. In contrast to atomistic and micromagnetic calculations, the spin-wave band structure was neglected as well (Djordjevic and Münzenberg, 2007). Such crude approximations have raised doubts about the adequateness of the approach (Walowski et al., 2008; Radu et al., 2009). It has recently been demonstrated that the model fails to describe the experimental results on demagnetization of the rare-earth-doped metals even qualitatively (Radu et al., 2009). In the follow up to this work (Koopmans et al., 2010) the spin-scattering mechanisms are described by a single dimensionless spin-flip parameter $a_{\text {sf }}$ that can be derived directly from experimental data. This allows the macroscopic demagnetization process to be related to microscopic spin-flip

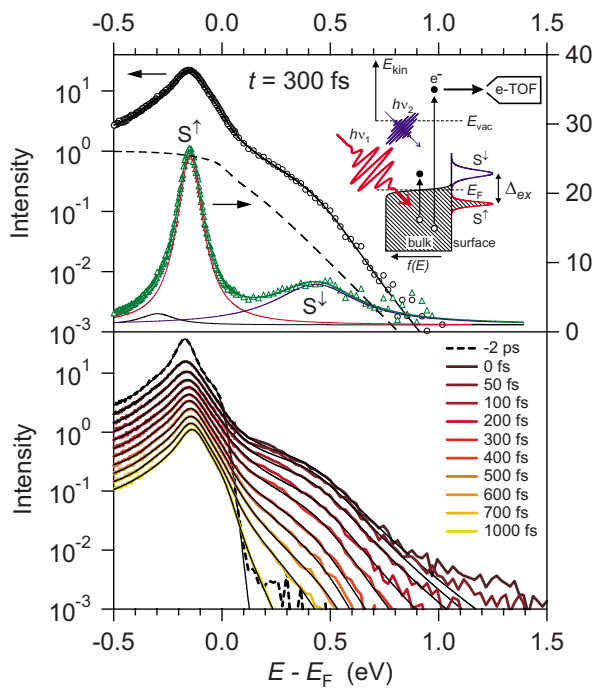

FIG. 9. (Color online) Time-resolved electron photoemission. Top: Normalized photoelectron spectrum at $300 \mathrm{fs}$ (circles) with fit (solid line) and distribution function $f(E ; t)$ (dashed line) on a logarithmic scale and divided by $f$ (triangles) on a linear scale at right. The inset sketches the TRPE experiment. Bottom: Photoelectron spectra at different delays are displayed in color (fits in black) and are offset vertically. From Lisowski et al., 2005.

processes. It will be interesting to see how this parameter $a_{\text {sf }}$ compares with the one extracted from spintransport experiments; see, e.g., Moreau et al. (2007).

\section{Interaction among charge, lattice, and spin subsystems}

\section{a. Time-resolved electron photoemission experiments}

Valuable information on the microscopic processes that accompany the ultrafast laser-induced demagnetization can be obtained by studying magnetic surfaces with surface-sensitive techniques. Such a low-dimensional structure reduces the phase space available for relaxation processes. As a consequence, relaxation rates are decreased compared to those in the bulk, facilitating a more detailed analysis. This advantage of surfaces has led in the last decade to a comprehensive understanding of the principles governing the decay of electronic excitations in metals (Echenique et al., 2004).

The $\operatorname{Gd}(0001)$ surface was found to be a favorable model system to study electron-phonon, electronmagnon, and phonon-magnon interactions and their respective dynamics by femtosecond time-resolved laser spectroscopy (Melnikov et al., 2003, 2008; Lisowski et al., 2005; Bovensiepen, 2007). The surface presents an exchange-split $5 d_{z^{2}}$ surface state with the majority spin component being occupied and the minority component unoccupied, respectively. Time-resolved photoemission (TRPE) was used to investigate the pump-induced variation in this exchange-split surface state. The top panel of Fig. 9 shows a spectrum around $E_{F}$ at 300 fs with the PE yield measured over five orders of magnitude. The occupied surface state $S^{\uparrow}$ is readily visible, while the unoccu- 
pied component $S^{\downarrow}$ shows up as a shoulder at $0.4 \mathrm{eV}$ above $E_{F}$. To fit the spectra two Lorentzians for $S^{\uparrow \downarrow}$, a constant bulk density of states, and a distribution function $f(E ; t)$ are used. Convolution with a Gaussian of $45 \mathrm{meV}$ width accounts for broadening of the laser pulse and the spectrometer resolution. As shown by Greber et al. (1997) and Rhie et al. (2003), unoccupied states can be analyzed with photoemission by normalizing the spectrum with a Fermi-Dirac distribution. On ultrafast time scales, nonthermalized electrons have to be included (Lisowski et al., 2004), which leads to small corrections of the Fermi distribution for Gd. After normalization to $f$, the surface contributions $S^{\uparrow}$ and $S^{\downarrow}$ are clearly discernible. Figure 9 shows that the optical excitation leads to an instantaneous population of $S \downarrow$ that decays within about 1 ps.

To develop a microscopic understanding of the observed ultrafast spin dynamics, two contributions should be considered: (i) secondary electrons in combination with transport processes and (ii) spin-flip scattering of hot electrons among spin-mixed states. The pump pulse excites $S^{\uparrow}$ electrons from the occupied surface state to unoccupied bulk states, minority electrons from bulk to unoccupied surface states, and transitions between bulk states. Transitions involving the surface state redistribute spin polarization from the surface to the bulk because majority electrons are excited into bulk states or minority electrons are excited to the surface state. This redistribution is a consequence of transport effects since the minority holes or majority electrons, which are excited in the vicinity of the surface, propagate into the bulk.

\section{b. Time-resolved $x$-ray experiments}

In an attempt to identify the angular momentum reservoir responsible for the disappearance of the spin order, Bartelt et al. performed studies of the laser-induced demagnetization of $\mathrm{Fe} / \mathrm{Gd}$ multilayers using timeresolved measurements of x-ray magnetic circular dichroism with 2 ps temporal resolution (Bartelt et al., 2007). Later Stamm et al. performed similar studies of ultrafast laser-induced demagnetization of $\mathrm{Ni}$ but with femtosecond temporal resolution and using femtosecond x-ray pulses (see Fig. 10) (Stamm et al., 2007). In both cases by separating spin and orbital contributions to the $\mathrm{XMCD}$, it was unambiguously demonstrated that the electron orbitals were not responsible for this fast demagnetization. Therefore the only possible reservoir should be the lattice. Because of the above-mentioned weak spin-lattice interaction, they argued that this can only be possible if some extra interaction, for example, via light-induced virtual states, enhances the spin-lattice interaction.

This finding seems to favor the Elliot-Yafet scattering as the most likely interaction mechanism. Indeed, electron-spin-wave scattering would keep the angular momentum inside the electron and spin systems, and only phonon- or impurity-induced scattering can transfer it to the lattice. Combining spin-, energy-, and time-

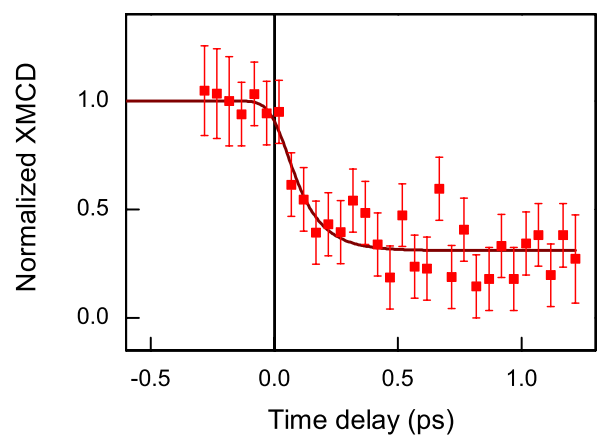

FIG. 10. (Color online) Time-resolved XMCD signal with circularly polarized $x$ rays incident at $60^{\circ}$ relative to the sample surface vs pump-probe time delay (symbols) measured at the $L_{3}$ edge maximum. As the measured value is proportional to $S+\frac{3}{2} L$, overall decrease in the signal excludes orbital moments from gaining any significant value at the cost of spins. From Stamm et al., 2007.

resolved two-photon photoemission with the timeresolved MOKE, Cinchetti et al. claimed to have found experimental evidence for the relevance of the ElliottYafet spin-flip processes for the ultrafast demagnetization on a time scale around 300 fs (Cinchetti et al., 2006).

How do these findings correlate with the generally accepted weak spin-lattice interaction? To answer this question, we recall the underlying physics. In general, spins couple to the electron orbital motion via spin-orbit coupling $U_{\mathrm{so}}=\lambda \mathbf{S} \cdot \mathbf{L}$, which is of the order of $50 \mathrm{meV}$. When placed in a crystal lattice, however, the orbits become distorted, so that the final states of the system are linear combinations of the original spherical harmonics. Such combinations are best viewed as standing waves of cylindrical symmetry with a periodic perturbation, and contain harmonics with opposite values of angular momentum. This interaction is in fact very strong, with typical values of the state splitting of the order of $1 \mathrm{eV}$. The exact value and sign of the splitting is given by the particular crystal symmetry. As a result of this interaction, the average orbital moment in each of the final states is zero or close to it (quenching of the orbital moments in transition metals). This leads to a strong reduction in the averaged value of the spin-orbit coupling in transitionmetal magnets which also leads to a weak static magnetocrystalline anisotropy.

Dynamically, however, the energy and angular momentum transfer from spins to lattice can be treated as a two-step process. In the first step, exchange of the energy and momentum between spins and orbitals occurs; in the second, it is the one between orbitals and lattice. At first it seems that such picture contradicts the observations by Stamm et al., who have not found any increase in the orbital angular momentum during the demagnetization process. Note, however, that in such twostep processes, the second step happens much faster than the first one so that any accumulation of orbital momentum is not to be expected. The transfer rate is thus determined by the slowest part, namely, the coupling between spins and the original atomiclike orbitals, 
around $50 \mathrm{meV}$, and thus occurs at a time scale of tens of femtoseconds.

Additional aspects of the role of angular momentum will be treated in Sec. VI.D.

\section{Microscopic models of ultrafast demagnetization}

$A b$ initio modeling has revealed possible mechanisms of ultrafast demagnetization without having the lattice involved. To achieve a control on demagnetization (and eventually magnetic switching), the electromagnetic field of the laser pulse was invoked as an active lever to drive the process (Zhang and Hübner, 2000). Also a model for ultrafast demagnetization of materials with discrete electronic structure was suggested (Gómez-Abal and Hübner, 2002) and all-optical subpicosecond spin switching was observed in ab initio simulations performed for $\mathrm{NiO}$ (Gómez-Abal et al., 2004). However, currently there is no experimental evidence that the mechanisms of the demagnetization and spin switching in $\mathrm{Ni}$ and $\mathrm{NiO}$ suggested by the $a b$ initio calculations are really taking place in these materials. Nevertheless, these works contained a number of fruitful ideas related to ultrafast coupling of light and matter. For instance, it was noted that switching by optical femtosecond pulses may follow a totally different trajectory, such as via a Raman-like twophoton excitation process.

An alternative approach to describe ultrafast demagnetization is based on an analysis of magnon-magnon and phonon-magnon interactions, while it neglects the electronic structure of a material. The classical micromagnetic modeling is performed for magnetic elements larger than $0.5 \mathrm{~nm}$, assuming a fixed length of the magnetization vector (macrospin) and using the LandauLifshitz-Gilbert equation (see Sec. II.A). Often an assumption of zero temperature is made; however, a stochastic variant of micromagnetic simulations allows one to take finite temperature into account (Berkov and Gorn, 1998; Fidler and Schrefl, 2000; Nowak et al., 2005). A modeling using the LLG equation cannot describe the processes in a magnetic system close to the Curie temperature, in general, and the phenomenon of ultrafast demagnetization, in particular. In this case the damping is enhanced when approaching the Curie temperature and the magnetization magnitude is not constant in time (Nowak et al., 2005). However, it is expected that an ultrafast laser excitation of free electrons in metals is followed by an electron-phonon interaction at the time scale of about 1 ps. Because of this interaction, the electron temperature decreases, bringing the magnetic system well below the Curie point. It is therefore reasonable to expect that micromagnetic simulations may provide an adequate modeling of the processes that occur in a spin system on a time scale longer than $1 \mathrm{ps}$ after laser excitation. An attempt to describe the processes following ultrafast laser excitation of a magnetic metal using such micromagnetic simulations was performed by Djordjevic and Münzenberg (2007) (Fig. 11). The model did not regard the details of the demagnetization and the quenching was assumed to be an instan-
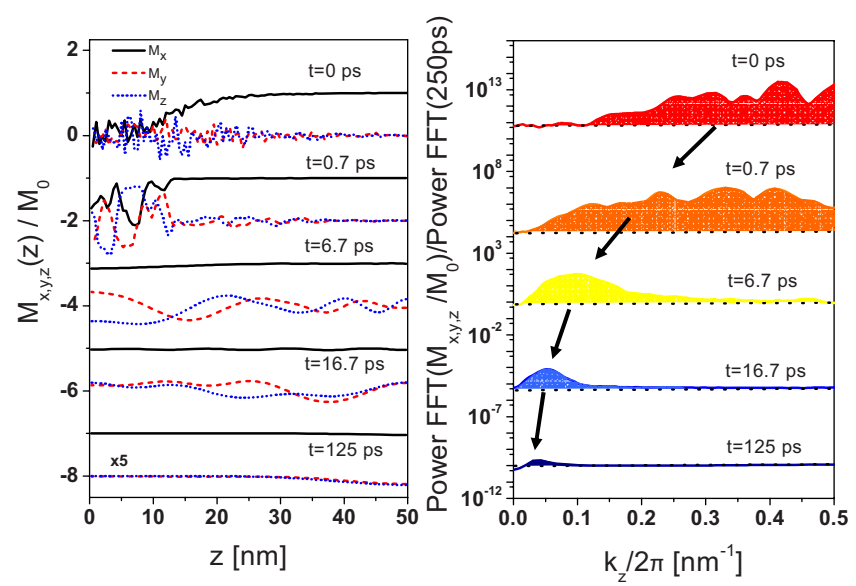

FIG. 11. (Color online) Cut through micromagnetic simulation for the Ni film after a partial demagnetization. Left: The evolution of spin-wave emission from the excited area shown in real space. Right: The corresponding Fourier transform shown as a function of spatial frequency. From Djordjevic and Münzenberg, 2007.

taneous process; it was described by orienting the magnetization vectors of $0.5 \mathrm{~nm}$ elements by a random angle (Eilers et al., 2006). From the micromagnetic simulations one sees that the instantaneous demagnetization is equivalent to the generation of a large number of magnons at the edge of the Brillion zone. In the following time domain the magnetization slowly recovers, and the speed of the recovery is defined by the speed of the energy transfer from the short-wavelength magnons to long-wavelength spin excitations via a spin-wave relaxation chain (i.e., magnon-magnon interaction). The results of the micromagnetic simulations based on the LLG equation were found in good agreement with timeresolved magneto-optical measurements of the laserinduced magnetization dynamics but only at time scales longer than 1 ps. For an adequate description of faster dynamics, a development of micromagnetic theory that is able to deal with elevated temperatures and macrospin dynamics beyond the LLG equation is necessary.

Another approach to modeling ultrafast laser-induced spin dynamics in a metallic magnet is to use the LLG equation on the atomic level (Chubykalo-Fesenko et al., 2006; Kazantseva, Nowak, et al., 2008). Essentially such modeling consists of the use of the Heisenberg model for the exchange coupling and the Langevin dynamic approach (the LLG equation augmented by a random field to include the effects of temperature) to describe the evolution of the ensemble of coupled spins. Ab initio calculations are used to provide information on the local properties such as the spin and exchange integral. A typical amount of modeled spins at the moment does not exceed $10^{6}$, corresponding to a maximum size of 20 $\times 20 \times 20 \mathrm{~nm}^{3}$. Nevertheless, the method appeared to be quite powerful for modeling magnetic systems in the vicinity of phase transitions (Chubykalo-Fesenko et al., 2006). For instance, it was able to reproduce an increase in the macroscopic transverse damping when the Curie temperature was approached. Similarly to micromag- 
netic simulations based on the LLG equation, the atomistic modeling was able to reproduce the recovery of the magnetization at the time scale of a few picoseconds (Kazantseva, Nowak, et al., 2008). Most importantly, the results of the simulations with the stochastic LLG equation on an atomistic level were found in excellent agreement with the experimental observations of a subpicosecond time scale for laser-induced demagnetization of a ferromagnet. Moreover, it was found that, even if energy pumped into the spin system at the subpicosecond time scale corresponds to heating above the Curie point, the system does not necessarily demagnetizes fully. Such a finding shows that for a spin system far from equilibrium the concept of spin temperature, often used for the description of ultrafast demagnetization (Beaurepaire et al., 1996), is not valid. It should be mentioned that microspin simulations based on the Landau-Lifshitz-Bloch equation seemed to capture the physics revealed by the atomistic model (Chubykalo-Fesenko et al., 2006) (see Sec. II.B). Finally, we note that it is becoming more obvious that for a solution of the problem of ultrafast demagnetization a multiscale approach, combining $a b$ initio, atomistic, and micromagnetic simulations, should be developed.

\section{B. Demagnetization of magnetic semiconductors}

The separation of spins and electrons into two separate baths (see Sec. IV.A.2) is a crude approach for treating itinerant ferromagnets, where no sharp separation between $s$ and $d$ bands is present. In contrast, such an approach is ideally suited for materials, where most of the macroscopic magnetization comes from the localized $d$ or $f$ shell spins, while the exchange interaction between the spins is mediated via itinerant $s$ carriers (Jungwirth et al., 2006). It is generally accepted that such a mechanism of exchange interaction is typical for rareearth metals such as Gd. At the same time, a similar mechanism of exchange is considered to be responsible for ferromagnetism in diluted magnetic semiconductors such as $\mathrm{EuO}, \mathrm{Ga}_{1-x} \mathrm{Mn}_{x} \mathrm{As}$, and $\mathrm{In}_{1-x} \mathrm{Mn}_{x}$ As.

In contrast to $\mathrm{EuO}$ and $\mathrm{EuS}$, fabrication of the novel (III,Mn)V ferromagnetic semiconductors only became possible not long ago. The first successful doping of $\mathrm{Mn}$ ions into InAs exceeding the heavy doping regime was reported by Munekata and co-workers in 1989 (Munekata et al., 1989). The subsequent discovery of ferromagnetism in $p$-type InMnAs films in 1991 triggered much interest in these materials (Ohno et al., 1992).

Ferromagnetism of (III,Mn)V semiconductors induced by relatively low concentrations of free carriers makes these materials unique model systems for the investigation of laser control of magnetic order. Unlike transition metals, in ferromagnetic semiconductors separation of the spins and electrons into two distinct subsystems is theoretically substantiated. And, unlike in rare-earth metals, the concentration of the free carriers responsible for the exchange between localized spins is relatively low and can be substantially controlled with the help of optically injected photocarriers.
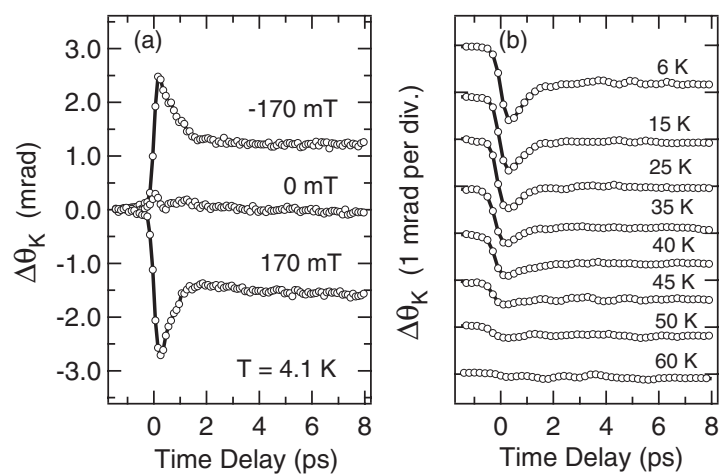

FIG. 12. Transient magneto-optical Kerr rotation in $\mathrm{Ga}_{0.974} \mathrm{Mn}_{0.026} \mathrm{As}$ triggered by a 140 fs laser pulse with a photon energy of $0.61 \mathrm{eV}$. (a) Magnetic-field dependence of the Kerr rotation. (b) Temperature dependence of the Kerr rotation between 6 and $60 \mathrm{~K}$. The signal decreases quickly with increasing temperature and is absent above the Curie temperature $T_{C}=50 \mathrm{~K}$. From Wang et al., 2006, 2008.

The first experiments on excitation of GaMnAs with subpicosecond laser pulses (the energy of the photons used was about $3.1 \mathrm{eV}$ ) revealed the feasibility of optical control of magnetism in these materials (Kojima et al., 2003). A photoinduced demagnetization process was observed at a time scale of $1 \mathrm{~ns}$, and no demagnetization at a subpicosecond time scale has been detected.

Later, however, experiments using time-resolved magneto-optical Kerr spectroscopy of ferromagnetic GaMnAs with mid-infrared pump pulses have allowed two distinct demagnetization processes to be revealed, a fast $(<1 \mathrm{ps})$ and a slow ( $\sim 100 \mathrm{ps})$ one; see Fig. 12 (Wang et al., 2006, 2008). While the slow component with a characteristic time of about $100 \mathrm{ps}$ is interpreted as a spin temperature increase via spin-lattice relaxation, an original concept was suggested for the interpretation of the time of the fast demagnetization processes (<1 ps) (Cywiński and Sham, 2007; Wang et al., 2008).

Since the energy of the photons used in the experiment $(0.61 \mathrm{eV})$ was insufficient for excitation of an electron from the valence to the conduction band, it was concluded that the observed demagnetization of GaMnAs resulted from the excitation of holes and a subsequent increase in their kinetic energy (temperature) (Wang et al., 2006, 2008) (Fig. 13). It was shown that the probability of a spin-flip process for $\mathrm{Mn}$ ions is enhanced with increasing effective hole temperature; the phenomenon was called the "inverse Overhauser effect" (Cywiński and Sham, 2007). It was noticed, however, that, if the demagnetization of the ferromagnet is not complete, the speed at which the magnetization drops might have no relation to the characteristic interaction time of the spins with other subsystems (electrons and phonons), see Fig. 13. Instead, the demagnetization time can be given by the characteristic time of the electron temperature drop. Since the probability for spin flip of Mn spins in the inverse Overhauser effect is proportional to the temperature of the free carriers, the fast cooling of the electron subsystem below a certain level 

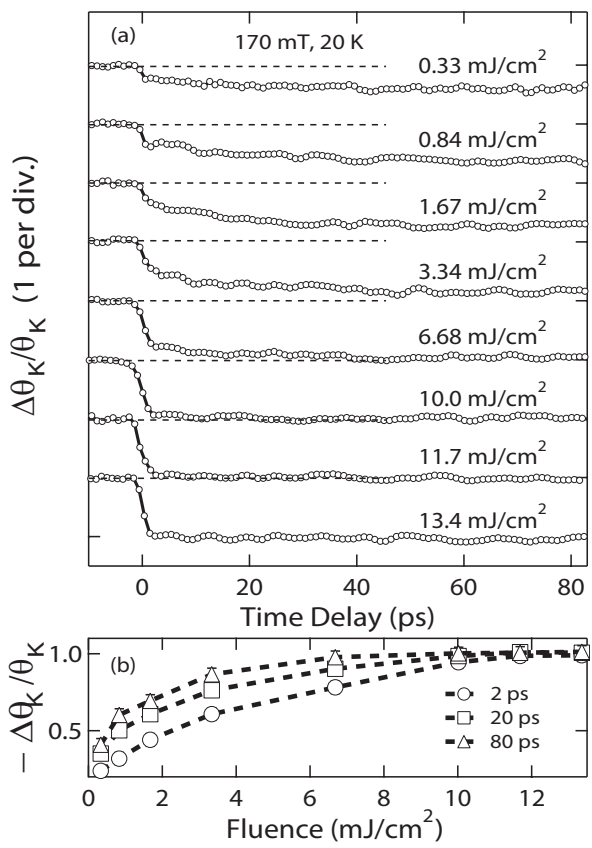

FIG. 13. Normalized photoinduced magneto-optical Kerr rotation in $\mathrm{In}_{0.87} \mathrm{Mn}_{0.13} \mathrm{As} / \mathrm{GaSb}$ heterostructure with Curie temperature $\sim 60 \mathrm{~K}$ (a) vs time for different pump fluences and (b) vs pump fluence for different time delays. At high fluences, the signal saturates to $\sim 1$, suggesting a complete quenching of ferromagnetic order. From Wang et al., 2005.

will effectively stop the demagnetization. Thus, the subpicosecond time scale of the demagnetization process may simply be the characteristic time of carrier-phonon interaction. It is important that there is again no electronic many-body interaction necessary. The connection between demagnetization time and energy relaxation of the excited carriers has been experimentally demonstrated in the study of the correlations between these two processes in GaMnAs (Wang et al., 2006, 2008).

We note, however, that an efficient angular momentum transfer from Mn spins to a hot hole gas is not enough to result in demagnetization since the $p$ - $d$ interaction conserves the total angular momentum. In addition to the redistribution of the angular momentum between the Mn spins and holes, an effective sink of the angular momentum from the holes to the lattice is required. Calculations show that short hole-spin relaxaion times of the order of $10 \mathrm{fs}$ are required for the explanation of ultrafast laser-induced demagnetization in GaMnAs and InMnAs (Cywiński and Sham, 2007; Morandi et al., 2009).

Finally, we note that the demagnetization of $(\mathrm{Ga}$, $\mathrm{Mn}$ )As can also be seen in an ultrafast change in coercivity (Hall et al., 2008) or terahertz emission (Héroux et $a l ., 2006)$. Terahertz emission induced by ultrashort laser excitation has also been observed in InMnAs. However, this was not associated with ultrafast demagnetization since the signal was also observed above the Curie point (Zhan et al., 2007).

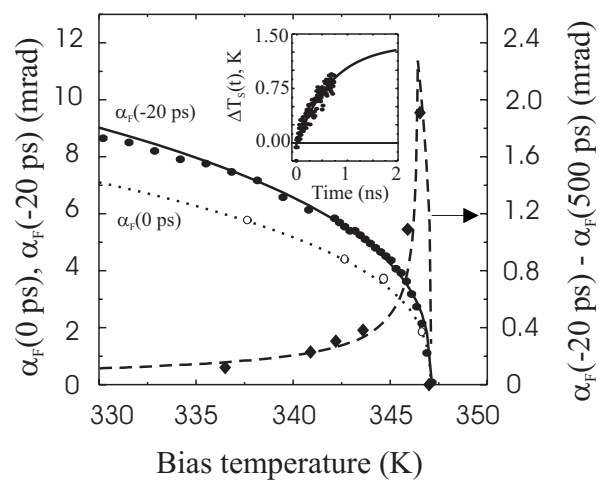

FIG. 14. The Faraday rotation at negative (solid circles) and zero (open circles) time delay as a function of the bias temperature with the fit to Eq. (23) (solid and dotted lines, respectively). The difference between the intrinsic magneto-optical signal and that at $500 \mathrm{ps}$ is shown by diamonds, together with the calculation based on the fitted parameters (dashed line). The inset shows the transient component of the magnon temperature as a function of the time delay. The solid line is the fit according to Eq. (24). From Kimel et al., 2002.

\section{Demagnetization of magnetic dielectrics}

It is instructive to compare the ultrafast demagnetization of ferromagnetic metals with a similar process in magnetic dielectrics. In the latter case, electrons are strongly localized and spins are located at the magnetic ions. Optical excitations in such a system rapidly relax via phonon cascades without affecting the magnetization, leading to an increase in the lattice temperature on a subpicosecond time scale. Only after this can the spin system be heated via phonon-magnon interaction. The collision integral between these quasiparticles is relatively small and is predominantly related to the spinorbit coupling in the magnetic ions, in the limited spectral range near the center of the Brillouin zone. It is thus no wonder that the typical times are of the order of a nanosecond.

To illustrate this, we consider here the laser-induced demagnetization of iron borate $\mathrm{FeBO}_{3}$ (Kimel et al., 2002). The detailed magnetic structure of this material is not important and will be considered below; see Sec. VI.D.1. In short, $\mathrm{FeBO}_{3}$ is a weak ferromagnet, i.e., the antiferromagnetically coupled spins are slightly tilted, leading to a net magnetization $M$ that can be probed via the Faraday effects in a pump-probe experiment.

The intrinsic Faraday effect in $\mathrm{FeBO}_{3}$ is shown in Fig. 14 as a function of temperature. Since the Faraday rotation is proportional to the order parameter, its temperature dependence is generally given by (Landau and Lifshitz, 1984)

$$
\alpha_{F}(T)=\alpha_{0}\left(1-T / T_{N}\right)^{\beta},
$$

where $T_{N}$ is the Néel temperature, $\beta$ is the critical exponent, and $T$ is the temperature of the spin system, which drives the order parameter. A fitting of Eq. (23) to the corresponding measurements in Fig. 14 results in $\beta$ $=0.364 \pm 0.008$ and $T_{N}=347.0 \pm 0.1 \mathrm{~K}$. These values are in 
good agreement with $\beta=0.354$ and $T_{N}=348.35 \mathrm{~K}$ as reported before (Wijn, 1994).

Figure 14 also shows the difference between the intrinsic magneto-optical signal and that at a time delay of 500 ps. This difference increases drastically before dropping to zero at the Néel point. This implies that the pump-induced relaxation of the magneto-optical signal is related to an increase in the magnon temperature. At a temperature of $T=346.5 \mathrm{~K}$ and a delay time of $500 \mathrm{ps}$, the Néel point is reached and magnetic order is destroyed.

Thus the magnetization dynamics is not directly affected by the optical excitation itself but instead is delayed considerably. In order to derive information about the dynamics from the measured transient Faraday rotation, the latter is converted into transient magnon temperatures $T_{s}(t)$ by means of Eq. (23) for all data below $T_{N}$. Decomposing these temperatures into a static temperature $T$ and an optically induced transient component $\Delta T_{s}(t)$, we found all $\Delta T_{s}(t)$ to be identical within the experimental error. Their average is shown in the inset of Fig. 14 and is characterized by a monotonic increase that was fitted by

$$
\Delta T_{s}(t)=T_{s}^{0}\left[1-\exp \left(-t / \tau_{\mathrm{sl}}\right)\right],
$$

where $T_{s}^{0}$ is the amplitude of the dynamical spin temperature and $\tau_{\mathrm{sl}}$ is the phonon-magnon interaction time. All variables were set as fitting parameters, and the result of the fit for $T_{s}^{0}=1.4 \mathrm{~K}$ and $\tau_{\mathrm{sl}}=700 \mathrm{ps}$ is shown in the inset by a solid line.

Using the deduced parameters $T_{s}^{0}, \tau_{\mathrm{sl}}$, and $\alpha_{0}$, the difference between the intrinsic Faraday rotation and that at a time delay of $500 \mathrm{ps}$ as a function of temperature (dashed line in Fig. 14) is directly derived. The excellent agreement with the experimental data supports the validity of such description.

Microscopically these results can be understood as follows. Because of the ${ }^{6} \Gamma_{1}^{+} \rightarrow{ }^{4} \Gamma_{4}^{+}$excitation, the electron potential energy increases by only $1.4 \mathrm{eV}$, while the excess of the photon energy is transferred either to the lattice or to the magnetic system. Generally, magnonassisted transitions are less intense than phonon-assisted ones (Tanabe and Aoyagi, 1982). Consequently, after the optical excitation the temperature of the phonons is higher than that of the magnons: $T_{l}>T_{s}$. This difference gradually vanishes and the magnon temperature increases with a time constant $\tau_{\mathrm{sl}}=700 \mathrm{ps}$ determined by the phonon-magnon interaction, which is predominantly related to the spin-orbit coupling in the magnetic ions (Kittel, 1958; Akhiezer et al., 1968).

A comparative study of different types of magnetic materials in the work of Ogasawara et al. (2005) showed that the relaxation time constant $\tau_{\mathrm{sl}}$ is strongly material dependent and scales with the magnetocrystalline anisotropy, indicating that the spin-orbit coupling is a dominant interaction for this process. It can also be understood in terms of the magnetic spin-wave frequency scaling with the anisotropy constant and thus accelerating all relevant processes.
Note that, although iron borate is an antiferromagnet, the presence of the weak ferromagnetic moment greatly facilitates the observation of the demagnetization dynamics. Similar experiments in pure antiferromagnets $\mathrm{Cr}_{2} \mathrm{O}_{3}$ (Satoh et al., 2007) are unavoidably connected with interpretation difficulties.

\section{Demagnetization of magnetic half metals}

A half metal is characterized by an electronic band structure that shows metallic behavior for one spin component, whereas it is a semiconductor (having a band gap) for the other. Experiments on laser-induced demagnetization of half-metallic materials therefore represent another intriguing opportunity to reveal a connection between electronic band structure and the dynamics of laser-induced demagnetization.

Laser-induced demagnetization was studied in the half-metallic ferromagnet $\mathrm{Sr}_{2} \mathrm{FeMoO}_{6}$ (Kise et al., 2000). An extremely slow relaxation of the spins on a time scale of about 500 ps was observed. It was claimed that this slow dynamics was due to the half-metallic nature of the material, where the spins are thermally insulated from the electron and lattice systems. In another half metal, $\mathrm{CrO}_{2}$, a similarly slow laser-induced demagnetization was observed and likewise explained by a vanishingly small interaction between electrons and spins typical for a half-metallic material (Zhang et al., 2006).

For ferromagnetic $(\mathrm{Ga}, \mathrm{Mn}) \mathrm{As}$, the observed slow spin dynamics upon laser-induced demagnetization was also explained as a consequence of the half-metallic band structure of this material (Kojima et al., 2003). After a rapid nonradiative relaxation, the subsequent carrier scattering occurs mainly near the Fermi surface. Therefore the demagnetization rate of carriers strongly depends on their spin polarization near the Fermi level. In particular, if the density of states for one spin direction is much smaller than that of the other direction, spin flips are inefficient, and spins and charges become thermally isolated, thus excluding the possibility of ultrafast laserinduced demagnetization. Later ultrafast demagnetization was demonstrated in both InMnAs (Fig. 13) and GaMnAs (Fig. 12), which seriously challenged this description in terms of half metallicity. The correlation between demagnetization time and spin polarization at the Fermi level has recently been studied systematically (Müller et al., 2009).

It should be mentioned, however, that the absence of ultrafast laser-induced demagnetization can have a much easier explanation. It has been noted in Sec. IV.C that insulating materials do not show ultrafast laser-induced demagnetization either. Because of the fundamental differences in the magnetic and transport properties of metals and insulators, the effect of a femtosecond pump pulse on these two types of magnetic material is different. In insulators, the effect of laser excitation is lattice heating, while in materials with wider energy bands, laser excitation leads to an increase in the kinetic energy of the itinerant electrons. As shown in Sec. IV.A, an increase in electron temperature can in principle lead to 


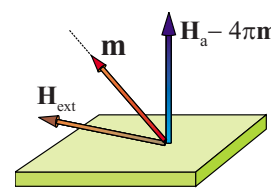

(a)

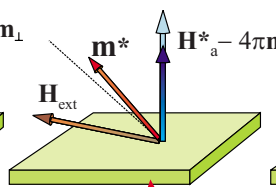

(b)

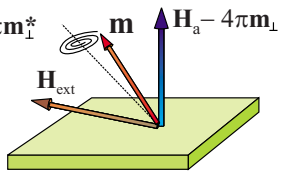

(c)
FIG. 15. (Color online) All-optical excitation of coherent precession. (a) $\Delta t<0$, the magnetization points in the equilibrium direction, given by the sum of the anisotropy, demagnetizing, and external fields; (b) $0<\Delta t<1 \mathrm{ps,} \mathrm{the} \mathrm{magnitude} \mathrm{of} M$ and/or the anisotropy change due to heating, thereby altering the equilibrium orientation; (c) the magnetization precesses around the new equilibrium direction that is slowly restored due to the heat diffusing away.

a rapid increase in spin temperature and consequently ultrafast demagnetization, while owing to the phononmagnon bottleneck, a heating of the lattice is followed by a much slower demagnetization at the subnanosecond time scale.

\section{E. Laser-induced coherent magnetic precession}

Ultrafast laser-induced changes of the magnetization can be exploited to provide an easy experimental method to study magnetic precession. For this purpose, an external field is applied in the near-hard-axis directions, in order to create a noncollinear geometry of the anisotropy, applied, and possibly also demagnetizing (shape anisotropy, $H_{d}=-4 \pi M_{\perp}$ ) fields [see Fig. 15(a)]. A rapid laser-induced change in the temperature and the magnetization value results in a change in the magnetocrystalline and/or shape anisotropies, and consequently in a change in the total effective field direction [Fig. 15(b)]. If this change is faster than the corresponding precession period, the magnetization vector will not be able to follow the net field and will start to precess around its new equilibrium [Fig. 15(c)]. Thus a noncollinear initial configuration of the various components of the net magnetic field is important so that the change in the value of the one component modifies the direction of the sum.

Time-domain measurements on the excited precession give quantitative information on the anisotropy, switching, and damping phenomena (Ju, Nurmikko, et al., 1998; van Kampen et al., 2002; Zhang, Nurmikko, et al., 2002; Vomir et al., 2005; Kimel et al., 2006; Rzhevsky et al., 2007; Langner et al., 2009). This technique can be used for a local probe of the magnetization dynamics in small structures. In addition, because of the nonuniform character of the excitation, standing spin waves can be excited, allowing for the investigation of spin-wave dispersion. Moreover, this technique is also well suited to study lateral standing waves, such as occur in nanostructures (Kruglyak, Barman, et al., 2005; Keatley et al., 2008).
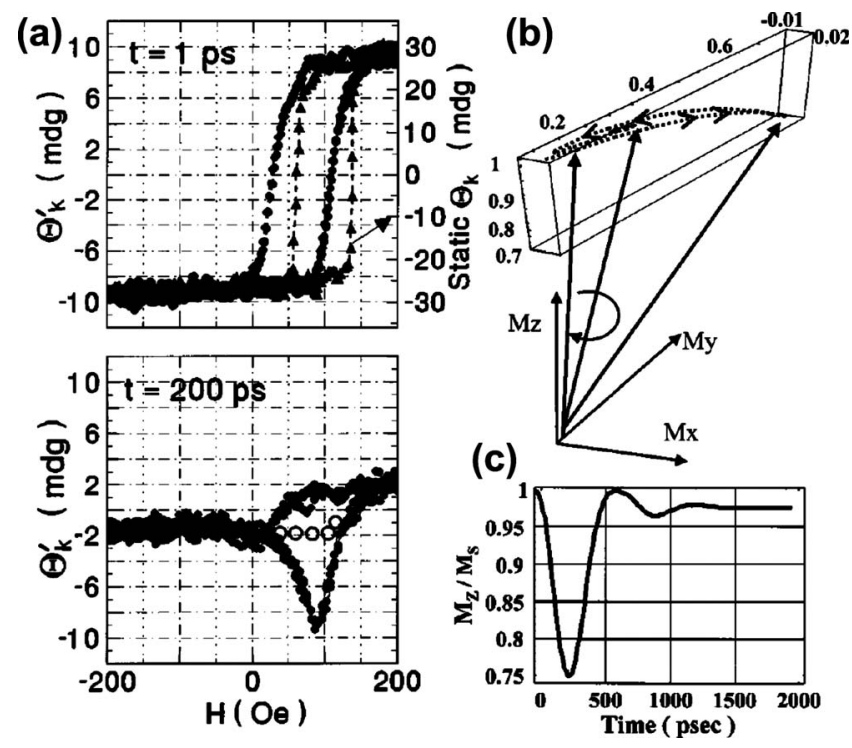

FIG. 16. Ultrafast laser-induced dynamics in $\mathrm{NiFe} / \mathrm{NiO}$ exchange-biased bilayer: (a) Easy-axis transient Kerr loops for the photoexcited sample at $t=1$ and $200 \mathrm{ps}$ following pulse photoexcitation at $t=0$. The open circles inserted in the bottom trace show expected behavior in the absence of coherent magnetization rotation. (b) Illustration of the trajectory of the magnetization vector in the typical LLG calculation. The initial direction of $M$ is directed along the $z$ axis. $M$ is subject to a damped rotation with a large $y$ component and a finite (outof-plane) $x$ component indicated schematically. The transient modulation of the $z$ component, measured by experiment, is projected out explicitly in (c). From Ju et al., 1999, 2000.

\section{Precession in exchange-biased bilayers}

As a specific test system, we consider the work of Ju et al., where an exchange-coupled $\mathrm{NiFe} / \mathrm{NiO}$ ferromagnetic (FM)/antiferromagnetic (AF) bilayer was studied (Ju, Nurmikko, et al., 1998; Ju et al., 1999, 2000). This bilayer is characterized by a distinct unidirectional magnetic anisotropy (Meiklejohn and Bean, 1956). The magnetic characteristics of such coupled FM/AF systems show both an effective exchange bias field $H_{\mathrm{ex}}$ (shifted hysteresis loop) and an increased coercivity $\left(H_{c}\right)$. The idea is to create spin excitations by laser excitation at the FM/AF interface by abruptly reducing the exchange coupling. When the magnetization of the FM layer is initially biased antiparallel to the external applied field $H$, the optically induced "unpinning" of the exchange would result in an ultrafast switching of the internal field $H_{\mathrm{ex}}$ and provide the driving force for the subsequent coherent magnetization dynamics. The low blocking temperature $T_{b} \sim 220{ }^{\circ} \mathrm{C}$ of the $\mathrm{NiO} / \mathrm{NiFe}$ system is convenient for breaking the exchange bias thermally.

Figure 16(a) shows "snapshots" of the easy-axis transient Kerr hysteresis loops acquired from a $100 \AA / 400 \AA \mathrm{NiFe} / \mathrm{NiO}$ bilayer sample at probe delays of $t \sim 1$ and $200 \mathrm{ps}$, following the pulsed laser excitation. The results are typical of several different samples studied and should be compared with the static (unperturbed) Kerr loop for the sample (dashed lines in the top 
panel). Within about 1 ps the transient Kerr loops show a significant reduction in the $H_{\mathrm{ex}}$. Some "softening" of the loop is seen, demonstrating direct electronic access to both the exchange coupling and the spins in the $\mathrm{NiFe}$ layer. On the other hand, the coercivity remains nearly unaffected.

With increasing time delay, the photoexcited, coupled FM/AF bilayer system relaxes via electron, spin, and lattice interactions. Over several tens of picoseconds, the transient Kerr loop, while reduced in amplitude due to these relaxation processes, begins to display a pronounced change in its shape. An asymmetric distortion sets in, the effect being concentrated in the lower right corner of the loop, which becomes quite dramatic at $t$ $\sim 200$ ps. Note that the detected signal is a measure of the pump-induced modulation of the magnetization and contains a contribution that is proportional to the changes in magnetization component in the plane of incidence, $\Delta M_{z}$. The large "negative Kerr loop anomaly" implies that a coherent magnetization rotation is triggered upon photoexcitation of the bilayer. When calibrated against the static Kerr rotation, this magnetization modulation induced by each pump laser pulse in Fig. 16(a), corresponds to $\Delta M_{z} / M_{s} \sim 0.4$ under these experimental conditions, or an average magnetization rotation of about $53^{\circ}$.

Figure 16(b) shows a pictorial view of the typical LLG simulation of the dynamical magnetization rotation process. The initial direction of the magnetization vector $\mathbf{M}$ is taken along the $z$ axis (in the plane of the thin film). Following the photoinduced change in the exchange bias field, $\mathbf{M}$ is subject to a damped rotation with a large $y$ component and a finite out-of-plane $x$ component indicated schematically in Fig. 16(b). The transient modulation of the $z$ component is projected out explicitly in Fig. 16(c).

\section{Precession in nanostructures}

Another example of light-induced magnetic precession concerns magnetic nanodots, where pump-probe measurements represent a real-time alternative to the spectroscopic tool such as Brillouin light scattering (Demokritov and Hillebrands, 2002).

The ground-state magnetization of a continuous thin film is virtually uniform. Consequently, the precession frequency is fully determined by the magnetic parameters of a sample and the value of the applied magnetic field (Gurevich and Melkov, 1996). In contrast, the internal magnetic field in finite-sized nonellipsoidal magnetic elements (such as used in practice) is nonuniform, which introduces additional complexity into the character of the observed magnetization dynamics. For example, the nonuniform demagnetizing field may lead to a spatial confinement and quantization of spin-wave modes on the nanometer length scale (Demokritov et al., 2001).

Here we illustrate this on an example of magnetic precession measured in a time-resolved Kerr-effect scheme from arrays of square Permalloy elements of different sizes in the nanometer range.

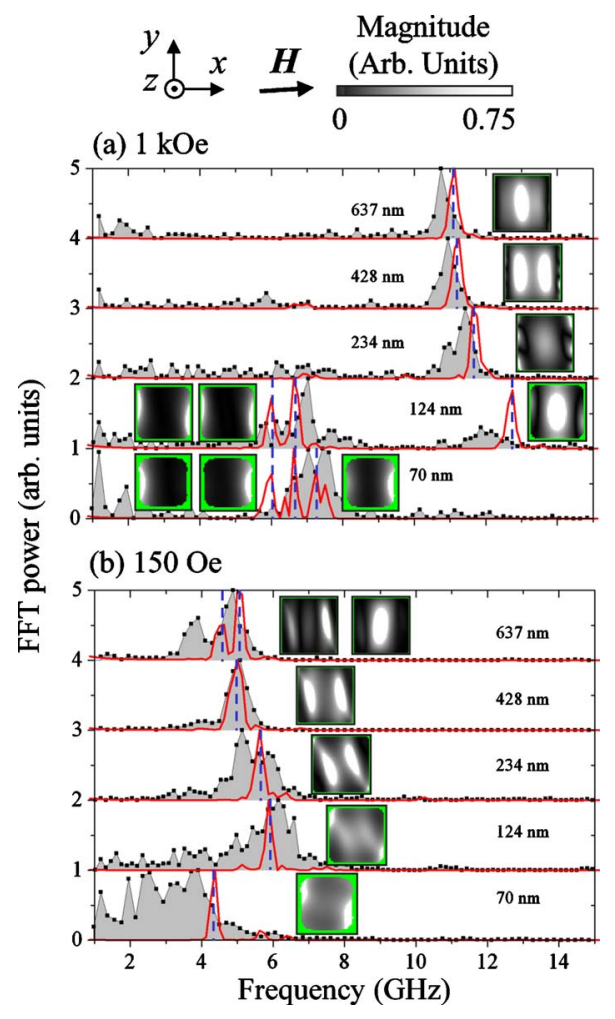

FIG. 17. (Color online) The dependence of the mode frequency upon the element size is shown for bias field values of (a) $1 \mathrm{kOe}$ and (b) $150 \mathrm{Oe}$. The shaded spectra were obtained experimentally while simulated spectra are shown with a solid curve. For each element size, the spatial distribution of the FFT magnitude of the modes in the simulated spectra is shown in the insets. From Keatley et al., 2008.

Fast Fourier transform (FFT) spectra calculated from the measured and simulated time-resolved signals are shown in Fig. 17 for two values of the bias field (a) 1 and (b) 150 Oe. At the bias field value of $1 \mathrm{kOe}$, the dependence of the experimental FFT spectra upon the size of the elements closely resembles that observed by Atxitia et al. (2009). As the element size is reduced from 637 to $236 \mathrm{~nm}$, the frequency of the dominant mode initially increases. At an element size of $124 \mathrm{~nm}$, at least two modes are clearly seen in both the experimental and simulated data. The "higher-frequency mode" continues the trend of increasing frequency as the element size is reduced; however, in the $124 \mathrm{~nm}$ element, its spectral power is reduced. The "lower-frequency mode" is separated from the higher-frequency mode by about $5 \mathrm{GHz}$ in the experimental spectra and has slightly higher spectral power. The frequency of the lower-frequency mode then increases as the element size is reduced further. The trend is reproduced by the simulated spectra. The spectra show a crossover in mode intensity for an element size of about $124 \mathrm{~nm}$.

Comparison of the measured spectra with simulations enables derivation of the spatial character of the observed precessional modes, as shown in the insets of Fig. 17. Modes can be classified according to their spatial character as center, edge, or detached edge modes. In 

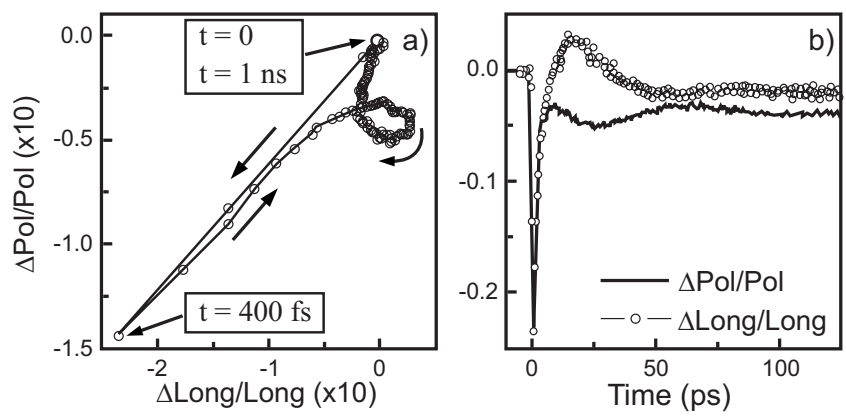

FIG. 18. Magnetization dynamics of $4 \mathrm{~nm}$ superparamagnetic cobalt particles in $\mathrm{Al}_{2} \mathrm{O}_{3}$. (a) Magnetization trajectory in the polar and longitudinal plane. (b) Dynamical polar and longitudinal Kerr signals (Andrade et al., 2006).

addition, because of the small $(<200 \mathrm{~nm})$ separation, interaction between the elements affects the dynamical behavior as well. A similar experimental and micromagnetic study of ultrafast laser-induced precession for nanostructured Ni films has been performed by Müller et al. (2008). Magnetic and elastic dynamics in periodic arrays of Permalloy nanodots induced by an ultrashort laser pulse has been investigated by Comin et al. (2006).

When the size of magnetic nanostructures is so small that their anisotropy energy $K V$ becomes comparable to the thermal energy $k T$, their magnetization will fluctuate between the two unidirectional states (Akharoni, 2000). This so-called superparamagnetic behavior results in a zero magnetization when it is averaged over a time scale larger than the typical time $\tau$ of these fluctuations. This fluctuation time $\tau$ generally varies over a very broad time scale, depending on the size of the particles. Although superparamagnetism was discussed by Néel in 1949 (Néel, 1949), it has recently become a real critical issue for magnetic storage where the bit sizes are becoming smaller and smaller.

Using $4 \mathrm{~nm}$ cobalt particles embedded in an $\mathrm{Al}_{2} \mathrm{O}_{3}$ matrix, Andrade et al. succeeded in visualizing the "Brownian" magnetization trajectories of superparamagnetic particles for the first time (Andrade et al., 2006). Obviously, for the investigation of such a motion the temporal resolution of the experiment has to be much better than the characteristic times investigated (typically a few picoseconds). Time-resolved magnetooptical measurements with subpicosecond temporal resolution is an elegant way to solve this problem. The particles were magnetized by an external field and then heated by a femtosecond laser pulse. The subsequent dynamics of the magnetization shown in Fig. 18 displays an oscillatory behavior characteristic of a strongly damped motion of precession. It is clearly present in the projection of the magnetization trajectory displayed in the longitudinal-polar plane up to 1 ns [see Fig. 18(a)]. Figure 18(b) shows the corresponding time-dependent differential polar and longitudinal components of the magnetization (displayed only up to $125 \mathrm{ps}$ ). The overall magnetization dynamics results from the ultrafast rise and subsequent decrease in the electron-spin temperature induced by the laser pump pulse, two processes which occur at time scales much faster than the motion of precession. In other words, the ultrafast change in temperature acts as a $\delta$ function excitation of the magnetization which induces a change in both its modulus and orientation. The reorientation of the magnetization vector is due to a dynamical change in the effective field related to the time-dependent anisotropy and exchange interactions, as shown recently in the case of thin cobalt films (Bigot et al., 2005). The precession frequency varies from 14 to $25 \mathrm{GHz}$ when $H$ varies from 2.1 to $3.3 \mathrm{kOe}$. It is seen that the maximum of the demagnetization at 400 fs coincides with the thermalization of the electrons. Next, a partial remagnetization occurs within 1 ps when the electrons cool down to the lattice via the electronphonon interaction. Simultaneously, the orientation of the magnetization changes and starts precessing.

The damping was found to be much larger in the nanoparticles than in the bulk and, in addition, it consistently increased when the particle size was decreased. The exact mechanisms of this enhanced damping observed in nanoparticles are still unknown but these results suggest that the metal-dielectric interface plays an important role to damp the precessional motion. It confirms previous studies of magnetization damping in $\gamma-\mathrm{Fe}_{2} \mathrm{O}_{3}$ or cobalt nanoparticles. Using ac susceptibility and Mössbauer spectroscopy measurements, Dormann et al. reported a value of $\alpha$ close to unity for $\gamma-\mathrm{Fe}_{2} \mathrm{O}_{3}$ particles (Dormann et al., 1996). Respaud et al. studied the ferromagnetic resonance of cobalt particles and reported values of $\alpha$ of 0.3 and 0.55 for particles containing, $\sim 310$ and $\sim 150$ cobalt atoms, respectively (Respaud et al., 1999). They attributed these large damping values to surface spin disorder.

\section{Precession in (III,Mn)As ferromagnetic semiconductors}

The recently fabricated novel ferromagnetic semiconducting alloys (III,Mn)As are materials the physical properties of which are poorly understood (Munekata $e t$ al., 1989; Ohno et al., 1992). During the last ten years, these materials have been intensively studied using various experimental techniques and methods. Several have reported time-resolved studies of laser-induced magnetic phenomena in $(\mathrm{Ga}, \mathrm{Mn})$ As semiconductors, demonstrating that excitation of these semiconductors with a subpicosecond laser pulse may trigger precession of the Mn spins at the frequency of the ferromagnetic resonance (Wang, Ren, et al., 2007; Hashimoto and Munekata, 2008; Hashimoto et al., 2008; Rozkotová et al., 2008a, 2008b; Kobayashi et al., 2009; Qi et al., 2009; Zhu et al., 2009). On the one hand, these observations have raised questions about the mechanism of such an all-optical excitation of the spin precession. On the other hand, the possibility of all-optical excitation of ferromagnetic resonance creates an intriguing opportunity to obtain extra information about the magnetic properties of the semiconducting alloys and their heterostructures and nanostructures.

It has been concluded by several research groups that the mechanism responsible for all-optical excitation of 
ferromagnetic resonance in $(\mathrm{Ga}, \mathrm{Mn}) \mathrm{As}$ semiconductors primarily relies on the laser-induced heating of the materials (Wang, Ren, et al., 2007; Rozkotová et al., 2008a; Qi et al., 2009). Because of absorption, an excitation of the semiconductor results in an effective energy transfer from light to the lattice. Because of the temperature dependence of the magnetic anisotropy of the materials, the heating changes the balance between different contributions of the magnetic anisotropy or affects the interplay between the magnetic anisotropy energy and the magnetostatic energy. Consequently, laser-induced heating changes the equilibrium orientation of the magnetization and if this change occurs much faster than the period of ferromagnetic resonance, it triggers the precession of the Mn spins.

It was observed that the polarization of the pump pulse hardly influenced the amplitude and phase of the spin oscillations (Wang, Ren, et al., 2007; Hashimoto et al., 2008; Qi et al., 2009). Moreover, the amplitude and frequency of the precession were found to be functions of the sample temperature (Rozkotová et al., 2008a; Kobayashi et al., 2009; Qi et al., 2009; Zhu et al., 2009). Both these observations are in agreement with the hypothesis that spin precession is excited via laser-induced heating. It is interesting to analyze the Gilbert damping of laser-induced spin precession in the annealed and asgrown samples (Qi et al., 2009). The Gilbert damping was seen to hardly depend on sample temperature or pump intensity in annealed samples. However, in the asgrown samples the damping increased with an increase in the pump intensity. This observation indicated a connection between the damping and $\mathrm{Mn}$-induced magnetic defects in GaMnAs. However, a theoretical analysis of the problem has not been performed yet.

A systematic analysis of the transient magneto-optical signals from GaMnAs ferromagnetic alloys was performed by Qi et al. (2009). It was shown that, if the photon energy of the pump pulse is below the band gap of GaMnAs $\left(E_{g}=1.53 \mathrm{eV}\right.$ at $\left.10 \mathrm{~K}\right)$, the photoinduced magneto-optical signals can be entirely attributed to ferromagnetically coupled spins. However, an above-bandgap excitation results in both $\mathrm{Mn}$ spin precession and pumping of electrons into the conduction band. The latter result in an additional contribution to the photoinduced magneto-optical Kerr effect, which, however, is weakly sensitive to temperature and insensitive to an external magnetic field. It was concluded that the electron spins are decoupled from the Mn spins (Qi et al., 2009).

A comprehensive analysis of the spin dynamics and magnetic eigenmodes excited by femtosecond laser pulses in $(\mathrm{Ga}, \mathrm{Mn}) \mathrm{As}$ slabs was performed by Wang, Ren, et al. (2007) yielding accurate values of the exchange, bulk, and surface anisotropy constants for this novel compound (see Fig. 19).

\section{Precession in ferrimagnetic materials}

Laser-induced coherent spin precession in systems where both magnetic moment and angular momentum
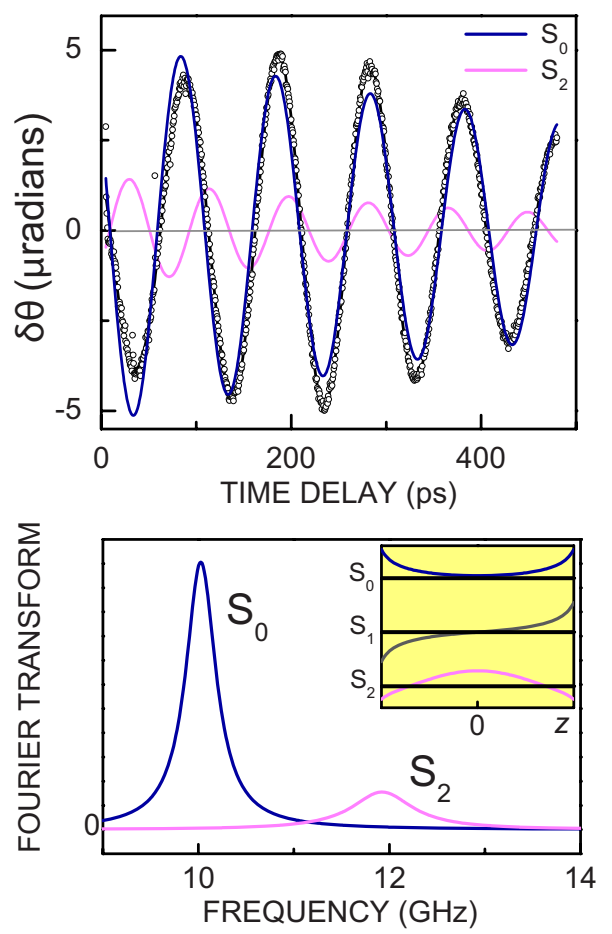

FIG. 19. (Color online) Light-induced magnetic precession in (Ga,Mn)As slabs. The top panel shows the transient magnetooptical Kerr signal measured in the Voigt geometry (open circles) at $H=0.17 \mathrm{~T}$. The applied field is parallel to [100]. The black curve is the linear prediction fit, which gives two modes of period of 99.7 and 83.9 ps. Their contributions to the fitted signal are shown by the curves. The bottom panel shows the Fourier transform of the fit and, in the inset, the calculated $M_{z}$ component for the three lowest eigenmodes. From Wang, Ren, et al., 2007.

can be continuously tuned, for example, by changing temperature, is a unique playground in which to study the role of the angular momentum in optical control of magnetism.

As one example of such a system, rare-earth-3d transition-metal (RE-TM) ferrimagnetic compounds were once widely used materials for magneto-optical (MO) recording. Depending on their composition, RE-TM ferrimagnets can exhibit a magnetization compensation temperature $T_{M}$ where the magnetizations of the RE and TM sublattices cancel each other and, similarly, an angular momentum compensation temperature $T_{A}$ where the net angular momentum of the sublattices vanishes. The theory of ferrimagnetic resonance (Wangsness, 1953, 1954) predicts a strong temperature dependence of the dynamic behavior in such systems. In particular, the frequency of the homogeneous spin precession as well as the Gilbert damping parameter $\alpha$ are expected to diverge at the temperature $T_{A}$ (Kobayashi et al., 2005). Such combination of a high frequency and large damping of the spin precession would provide a means for ultrafast and ringing-free magnetization reversal via precessional motion (Schumacher et al., 2003).

In a ferrimagnetic system, the Landau-Lifshitz-Gilbert equation should be written for each $i$ th sublattice 
$(i=\mathrm{RE}, \mathrm{TM})$. These equations are coupled by the presence of the exchange field $\mathbf{H}_{\mathrm{RE}, \mathrm{TM}}^{\mathrm{ex}}=-\lambda_{\mathrm{ex}} \mathbf{M}_{\mathrm{TM}, \mathrm{RE}}$ and give rise to two modes. Here $\lambda_{\mathrm{ex}}$ is a parameter that characterizes the strength of the exchange field. The ferromagnetic mode with $\omega_{\mathrm{FMR}}=\gamma_{\mathrm{eff}} \mathbf{H}^{\mathrm{eff}}$ can be described by a single LLG equation but now employing an effective gyromagnetic ratio $\gamma_{\text {eff }}$ (Kittel, 1949; Wangsness, 1953),

$$
\gamma_{\mathrm{eff}}(T)=\frac{M_{\mathrm{RE}}(T)-M_{\mathrm{TM}}(T)}{M_{\mathrm{RE}}(T) /\left|\gamma_{\mathrm{RE}}\right|-M_{\mathrm{TM}}(T) /\left|\gamma_{\mathrm{TM}}\right|}=\frac{M(T)}{A(T)},
$$

and an effective Gilbert damping parameter $\alpha_{\text {eff }}$ (Mansuripur, 1995),

$$
\alpha_{\mathrm{eff}}(T)=\frac{\lambda_{\mathrm{RE}} /\left|\gamma_{\mathrm{RE}}\right|^{2}+\lambda_{\mathrm{TM}} /\left|\gamma_{\mathrm{TM}}\right|^{2}}{M_{\mathrm{RE}}(T) /\left|\gamma_{\mathrm{RE}}\right|-M_{\mathrm{TM}}(T) /\left|\gamma_{\mathrm{TM}}\right|}=\frac{A_{0}}{A(T)}
$$

where $M(T)$ and $A(T)$ are the net magnetic moment and net angular momentum, respectively; $\lambda_{\mathrm{TM}}$ and $\lambda_{\mathrm{RE}}$ are the Landau-Lifshitz damping parameters for the rareearth and transition-metal sublattices, respectively; $A_{0}$ is a constant under the assumption that the LandauLifshitz damping parameters are independent of temperature (Kobayashi et al., 2005). The validity of this assumption was confirmed over a wide temperature interval by FMR measurements in $3 d$-TM (Bhagat and Lubitz, 1974).

In addition to the ferromagnetic mode $\omega_{\mathrm{FMR}}$, spins in a ferrimagnetic system may oscillate with the exchange resonance frequency (Kaplan and Kittel, 1953)

$$
\begin{aligned}
\omega_{\mathrm{ex}} & =\lambda_{\mathrm{ex}}\left(\left|\gamma_{\mathrm{TM}}\right| M_{\mathrm{RE}}-\left|\gamma_{\mathrm{RE}}\right| M_{\mathrm{TM}}\right) \\
& =\lambda_{\mathrm{ex}}\left|\gamma_{\mathrm{RE}} \| \gamma_{\mathrm{TM}}\right| A(T) .
\end{aligned}
$$

Equations (25) and (26) indicate a divergence of both the precession frequency and the Gilbert damping parameter of the FMR mode at the temperature $T_{A}$. Moreover, from Eq. (25), one notices that at the temperature $T_{M}$ the FMR frequency becomes zero. In contrast, Eq. (27) indicates that the exchange resonance branch softens at the angular momentum compensation temperature $T_{A}$ (Wangsness, 1955), where the FMR mode diverges.

Laser-induced spin precession in the ferrimagnetic GdFeCo alloy was investigated using a pump-and-probe method (see Secs. II.A and II.B) (Stanciu et al., 2006). The sample used in these experiments was a 20-nm-thick GdFeCo layer, a ferrimagnetic amorphous alloy with perpendicular magnetic anisotropy. Strong coupling between the RE and TM subsystems leads to a common Curie temperature $\left(T_{C}\right)$ which for the given alloy composition $\left(\mathrm{Gd}_{22} \mathrm{Fe}_{74.6} \mathrm{Co}_{3.4}\right)$ is about $500 \mathrm{~K}$.

Figure 20 shows the temperature dependence of (a) the magnetization precession frequency and (b) the Gilbert damping parameter. At $T=220 \mathrm{~K}$ a significant increase is observed in both the precession frequency and the damping parameter. As expected from Eqs. (25) and

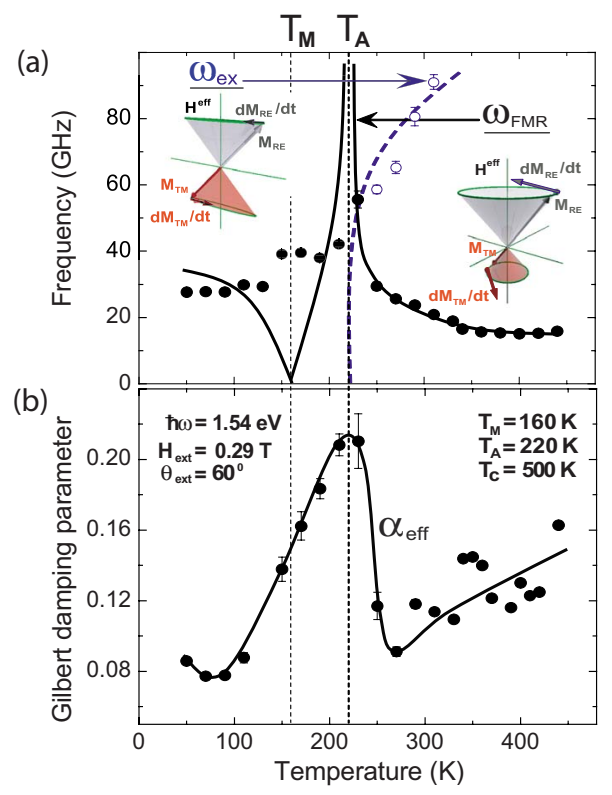

FIG. 20. (Color online) Temperature dependence of (a) the magnetization precession frequencies $\omega_{\mathrm{FMR}}$ and $\omega_{\mathrm{ex}}$. As temperature decreases from $310 \mathrm{~K}$ toward $T_{A}$, the exchange resonance mode $\omega_{\text {ex }}$ (open circles) softens and mix with the ordinary FMR resonance $\omega_{\mathrm{FMR}}$ (closed circles). The solid lines are a qualitative representation of the expected trend of the two resonance branches as indicated by Eqs. (25) and (27). (b) Temperature dependence of the Gilbert damping parameter $\alpha_{\text {eff }}$. The lines are guides to the eye. From Stanciu et al., 2006.

(26), the fact that both $\alpha_{\text {eff }}$ and $\omega_{\text {FMR }}$ peak at the same temperature indicates the existence of angular momentum compensation near this temperature of $220 \mathrm{~K}$. The strong temperature dependence of $\gamma_{\text {eff }}$ demonstrates the nonequivalent character of the gyromagnetic ratios of the two magnetic sublattices in $\mathrm{GdFeCo}$. This inequivalence also leads to the difference between the point of magnetization compensation $T_{M}$ and angular momentum compensation $T_{A}$. In addition to the peak near $T_{A}$, an enhancement of $\alpha_{\text {eff }}$ has also been observed as the temperature is increased toward $T_{C}$. Again Eq. (26) predicts this enhancement under the assumption of temperature-independent Landau-Lifshitz damping parameters $\lambda_{\mathrm{RE}}$ and $\lambda_{\mathrm{TM}}$. This enhancement is consistent with earlier data ( $\mathrm{Li}$ and Baberschke, 1992; Silva et al., 2004). These measurements demonstrate the consistency of the theoretical prediction of Eq. (26) with the temperature dependence of $\alpha_{\text {eff }}$ in RE-TM alloys such as $\mathrm{GdFeCo}$. The observed strong increase in the precession frequency and the Gilbert damping when the temperature approaches $T_{A}$ is ideal for ultrafast ringing-free precessional switching in magnetic and magneto-optical recording.

In the temperature region just above $T_{A}$, Fourier analysis of the measured time dependencies reveals two frequencies, one decreasing and the other increasing with temperature. While the former can be attributed to the FMR mode, the temperature behavior allows clear identification of the latter as the exchange mode [see 
Eq. (27)], the frequency of which can be low around $T_{A}$ but is usually very high everywhere else.

\section{F. Laser-induced phase transitions between two magnetic states}

Ultrafast laser-induced heating can also be used to trigger magnetic phase transitions on a (sub)picosecond time scale. Soon after the discovery of ultrafast demagnetization in $\mathrm{Ni}$, Agranat et al. investigated the ultrafast dynamics of first- and second-order magnetic phase transitions in a TbFeCo film (Agranat et al., 1998). At room temperature the studied compound was in an amorphous ferrimagnetic phase with the easy axis of magnetic anisotropy perpendicular to the film. However, heating led to a drastic change in the magnetic and structural properties of the film. At a temperature $T_{C 1}$ $\approx 420 \mathrm{~K}$, the material undergoes a second-order phase transition into an amorphous paramagnetic state. At $T_{a c} \approx 570 \mathrm{~K}$ a first-order and a second-order phase transition occur, causing both a crystallization and the formation of a magnetic state with the magnetization in the plane of the sample. This TbFeCo film was excited by an intense picosecond laser pulse. It was found that a laserinduced heating up to temperatures $T_{C 1}<T<T_{a c}$ resulted in a demagnetization within $5-10$ ps. A similar demagnetization was observed if the laser brought the material up to $T>T_{C 2}$, where $T_{C 2} \approx 720 \mathrm{~K}$ is the Curie temperature of the crystalline phase. Surprisingly, if the laser intensity was tuned such that the film was heated up to $T_{a c}<T<T_{C 2}$, the crystallization and spin reorientation occurred within 1 ps directly, i.e., without the formation of the amorphous paramagnetic phase. Although it is clear that demagnetization of $\mathrm{TbFeCo}$ alloys may take place at a much shorter time scale (Kim et al., 2009), these experiments raise fundamental questions about the mechanisms and ultimate speed of phase transitions between two magnetic states.

\section{Spin reorientation in $\mathrm{TmFeO}_{3}$}

In antiferromagnetic $\mathrm{TmFeO}_{3}$, as the temperature is lowered a spontaneous spin reorientation occurs as a result of a strong temperature dependence of the magnetic anisotropy (Belov et al., 1969; White, 1969; Wijn, 1994). In this process, the antiferromagnetic vector $I$ turns continuously from its position along the $x$ axis at a temperature $T_{2}$ to a position along the $z$ axis at a temperature $T_{1}$.

The temperature-dependent anisotropy energy in $\mathrm{TmFeO}_{3}$ has the form (Horner and Varma, 1968; Shapiro et al., 1974)

$$
F(T)=F_{0}+K_{2}(T) \sin ^{2} \theta+K_{4} \sin ^{4} \theta,
$$

where $\theta$ is the angle in the $x-z$ plane between the $x$ axis and the antiferromagnetic (AM) moment $\mathbf{I}$ and $K_{2}$ and $K_{4}$ are the anisotropy constants of second and fourth orders, respectively. Application of equilibrium conditions to Eq. (28) yields three temperature regions corresponding to different spin orientations:

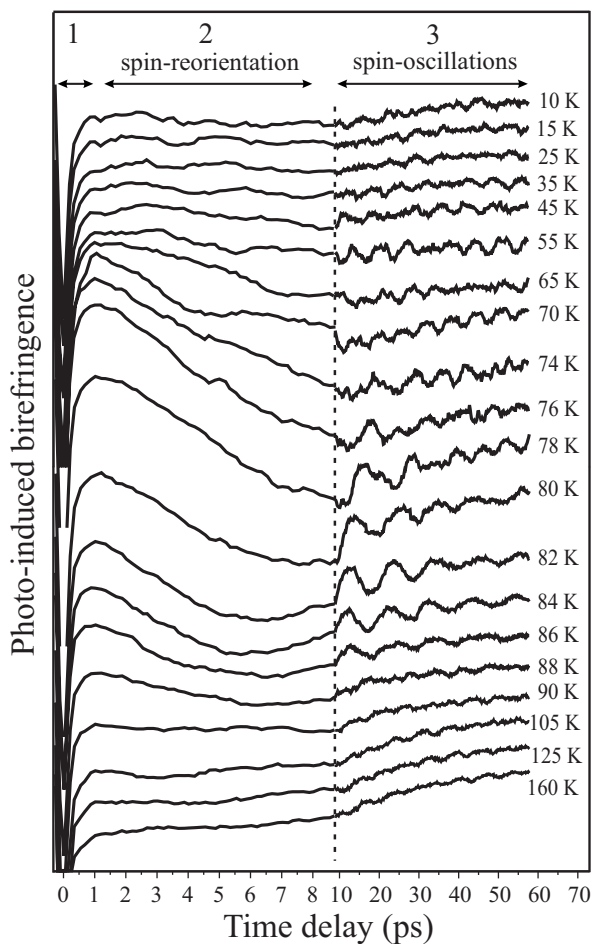

FIG. 21. Excitation and relaxation of the AFM vector moment measured via changes in the magnetic birefringence. Here one can distinguish three processes: (1) electron-phonon thermalization with 0.3 ps relaxation time, (2) rotation of the AFM vector with 5 ps response time, and (3) oscillations of the AFM vector around its equilibrium direction with an approximate 10 ps period. From Kimel, Kirilyuk, et al., 2004.

$$
\begin{aligned}
& \Gamma_{4}\left(G_{x} F_{z}\right): \quad \theta=0, \quad T \geqslant T_{2}, \\
& \Gamma_{2}\left(G_{z} F_{x}\right): \quad \theta=\frac{1}{2} \pi, \quad T \leqslant T_{1}, \\
& \Gamma_{24}: \quad \sin ^{2} \theta=-\frac{K_{2}(T)}{2 K_{4}}, \quad T_{1} \leqslant T \leqslant T_{2},
\end{aligned}
$$

where $T_{1}$ and $T_{2}$ are determined by the conditions $K_{2}\left(T_{1}\right)=-2 K_{4}$ and $K_{2}\left(T_{2}\right)=0$. Here $\Gamma s$ indicate the representations of the respective symmetry groups (Koster and Statz, 1963). Therefore, depending on the anisotropy constants, a spin reorientation may be expected that shows two second-order phase transitions at $T_{1}$ and $T_{2}$. The temperature dependence of $\theta$ in the phase $\Gamma_{24}$ is determined by $K_{2}(T)$, which varies roughly linearly with temperature (Belov et al., 1969).

The laser-induced transition between the two spin configurations in this antiferromagnet can be monitored with the help of linear optical birefringence (see Sec. III.B). The resulting changes in the birefringence are summarized in Fig. 21 (Kimel, Kirilyuk, et al., 2004). In the time domain, the relaxation process can be divided in three distinct regions. First, the excitation of hot electrons decays via phonon cascades and the phonon system thermalizes in a very short time (process 1 in Fig. 21, with a time constant of around $0.3 \mathrm{ps}$ ). The phonon- 
phonon interaction sets a new lattice temperature so that the equilibrium anisotropy axis is changed. Under such conditions, the magnetization vector in a FM material would precess around its new equilibrium direction, approaching it due to damping (Landau and Lifshitz, 1935). In an antiferromagnet, the exchangecoupled spins start to precess in opposite directions, thus creating a strong exchange torque $\mathbf{T}_{\mathrm{ex}}$ that opposes this precession. The resulting motion of the spins to the new equilibrium should then occur in the plane spanned by the anisotropy field $\mathbf{H}_{A}$ and spins $\mathbf{S}$. This process is marked 2 in the time dependencies of Fig. 21 and has a characteristic time of about 4 ps. This relaxation time corresponds to an AM resonance frequency of $80 \mathrm{GHz}$. The amplitude of this spin reorientation reaches $30^{\circ}$.

After the initial relaxation, the antiferromagnetic vector oscillates around its new equilibrium (process 3 ). Particularly strong oscillations are observable in the range of $80-90 \mathrm{~K}$, i.e., in the region of the reorientational transition. In fact, the derived frequencies closely resemble these of the spin waves with $k=0$ (Shapiro et al., 1974; White et al., 1982). Such spin waves are equivalent to a homogeneous precession of the magnetization observed at such conditions in ferromagnets (van Kampen et al., 2002).

The experimental results show that the AM spins in $\mathrm{TmFeO}_{3}$ are reoriented by several tens of degrees within only a few picoseconds. For comparison, in a ferromagnet with an anisotropy energy similar to that in $\mathrm{TmFeO}_{3}\left[10^{4} \mathrm{~J} / \mathrm{m}^{3}\right.$ (Wijn, 1994) $]$ the magnetization precesses with a period of several hundred picoseconds (Gerrits et al., 2002; Schumacher et al., 2003).

The dynamics of a similar laser-induced phase transition has been investigated in $\mathrm{Fe} / \mathrm{Gd}$ multilayers with the help of element-specific XMCD with temporal resolution of about 80 ps (Eimüller et al., 2007). Surprisingly, the magnetic relaxation was found to be noticeably slower than the cooling by heat diffusion. It was speculated that this slow relaxation can be attributed to the weak magnetic anisotropy.

\section{Spin reorientation in (Ga,Mn)As}

The ferromagnetic semiconductor $(\mathrm{Ga}, \mathrm{Mn}) \mathrm{As}$ has a biaxial magnetic anisotropy (Jungwirth et al., 2006) and as a result possesses two equivalent easy axes. This property modifies the hysteresis loop such that a switching of the magnetization between two different pairs of states can be observed. It has been demonstrated that the switching can be triggered by laser-induced heating. Owing to the temperature dependence of the fourfold magnetic anisotropy of this magnetic alloy, excitation of the material with a single subpicosecond light pulse leads to spin rotation over $90^{\circ}$ in the plane of the sample (Astakhov et al., 2005). To monitor such a reorientation the phenomenon of magnetic linear birefringence was used (see Sec. III.B for details). In particular, the angle of incidence was around $20^{\circ}$ to the structure growth direction and the incoming polarization was set at $45^{\circ}$ with respect to the [100] axis. A magnetic field was applied in

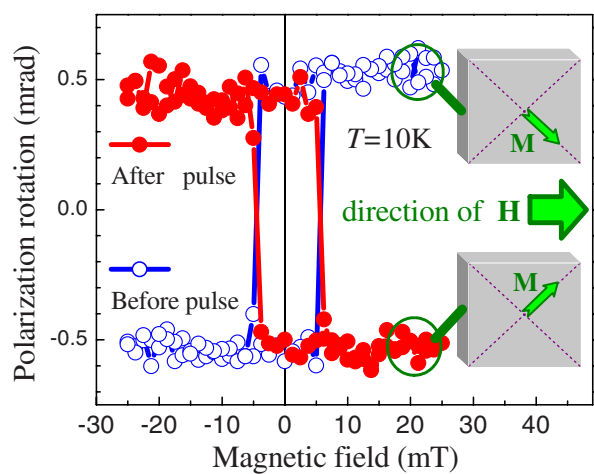

FIG. 22. (Color online) Minor hysteresis loops of the $\mathrm{Ga}_{0.98} \mathrm{Mn}_{0.02} \mathrm{As}$ sample, recorded before (open symbols) and after (solid symbols) excitation by single optical pulse with a duration of $100 \mathrm{fs}$, shown together with corresponding magnetization orientations (insets). From Astakhov et al., 2005.

the plane of the sample at an angle of $42^{\circ}$ with respect to the [100] axis. Amplified 100 fs pulses from a Ti:sapphire laser at a repetition rate of $1 \mathrm{kHz}$ at a wavelength $805 \mathrm{~nm}$ were used for the ultrafast laser excitation of the sample. A mechanical shutter was used to select one single pulse. This pulse was focused on the sample to a spot size of $100 \mu \mathrm{m}$. The pump fluence was up to $150 \mathrm{~mJ} / \mathrm{cm}^{2}$. Excitation of the sample with such a laser pulse led to the spin reorientation over $90^{\circ}$. In the geometry of the experiment such an reorientation was seen as a reversal of the magnetic hysteresis loop (see Fig. 22). This observation demonstrates that ultrafast laser excitation is able to induce far more drastic changes than just a switch in the orientation of the magnetization. In the present experiment, the hysteresis loop as a whole is completely reversed. It is also demonstrated that by applying ultrashort optical pulses we can manipulate the magnetization in a $(\mathrm{Ga}, \mathrm{Mn}) \mathrm{As}$ layer between its four metastable states, which can be used for application in a magneto-optical memory.

\section{AM-to-FM phase transition in FeRh}

Recently two groups independently showed the possibility of generating ferromagnetic order at a subpicosecond time scale in FeRh thin films (Ju et al., 2004; Thiele et al., 2004). FeRh is a particularly interesting system that displays an antiferromagnetic phase below and a ferromagnetic phase above a phase transition temperature around $370 \mathrm{~K}$. Laser heating of such a film, driving it through its AM-to-FM phase transition, showed a first onset of ferromagnetism within the first picosecond, which implied that the phase transition was of electronic origin. On a longer time scale a continued growth of the ferromagnetic order was witnessed, possibly due to domain expansion. Not only does FeRh provide a unique model system for improving our theoretical understanding of (sub)picosecond magnetic processes, it also receives considerable interest for its potential application in heat-assisted magnetic recording (Thiele et al., 2003).

Time-resolved MOKE measurements were made on $\mathrm{Fe}_{45} \mathrm{Rh}_{55}$ thin film samples (thickness $100 \mathrm{~nm}$ ), made 


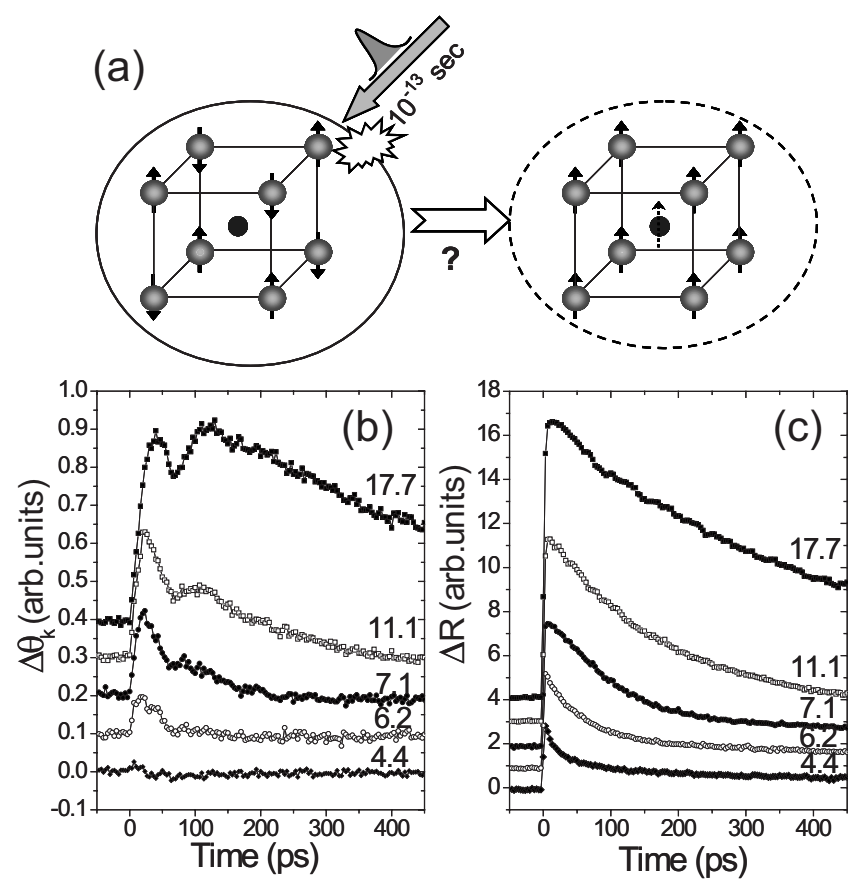

FIG. 23. Ultrafast laser-induced dynamics in RhFe: (a) Schematic of the ultrafast generation of ferromagnetic order by inducing an AM-FM transformation in FeRh when excited with femtosecond optical pulses. Time evolution of (b) the transient Kerr effect $\Delta \theta_{K}(t)$ and (c) the transient reflectivity $\Delta R(t)$ as a function of pump fluences (labeled for each curve in $\left.\mathrm{mJ} / \mathrm{cm}^{2}\right)$. The curves are vertically displaced for clarity. From Ju et al., 2004.

with dc-magnetron sputtering on a $\mathrm{MgO}$ substrate. In Fig. 23(b) the Kerr rotation during the first 700 ps after pump excitation of a $100 \mathrm{~nm}$ FeRh film is presented for increasing pump powers. A clear threshold behavior is observed. A minimum fluence of $\sim 6 \mathrm{~mJ} / \mathrm{cm}^{2}$ is found to be required to pass the transition temperature, and only for higher powers is a finite laser-induced Kerr rotation observed. We stress that the observation of a threshold power is considered as an essential fingerprint of the laser-induced phase transition. As a second signature of driving the film into the ferromagnetic phase, the duration over which the induced Kerr signal lasts increases as a function of pump fluence (up to nanoseconds at the highest fluences), because it takes increasingly longer before the magnetic film is cooled down below the phase transitions by diffusive cooling.

Time-resolved MOKE and transient reflectivity data were compared with simulations that included magnetization growth and alignment processes (Bergman et al., 2006). The initial rapid growth of magnetic moment within about 10 ps was followed by a gradual alignment process where local magnetic moments are aligned relative to each other in about 50 ps. The observed magneto-optical transients demonstrated an interplay among the local magnetic moment, nonlocal domain growth or alignment, and magnetization precession which was launched by the varying demagnetizing fields.

\section{G. Magnetization reversal}

Laser-induced heating of a magnetic medium in the presence of an external field is used in thermomagnetic writing to record a bit of information via magnetization reversal. Revealing the ultimate speed at which such a recording event can be realized is a fundamentally interesting issue with possible consequences for the future development of magnetic recording and information processing. Fast magnetization reversal induced by femtosecond laser pulses was investigated in $\mathrm{GdFeCO}$ alloys (Hohlfeld et al., 2001). In the experiment an external magnetic field directed antiparallel to the magnetization and a strength smaller than the coercive field was applied. Femtosecond laser excitation of the sample led to a total demagnetization of the material and a slow recovery of the magnetic order with the magnetization reversed. Although the demagnetization occurred at the subpicosecond time scale, the medium remained in the demagnetized state for a few picoseconds (Kazantseva, Nowak, et al., 2008) and then exhibited slow recovery of the magnetization, which was completed in about 750 ps. Moreover, comparison of temperature-induced dynamics and of field-induced magnetization reversal to data obtained for the same high pump fluence in remanence demonstrated that the dynamics of remanent magnetization cannot be interpreted by temperature dynamics only, and the dynamics of magnetic domains should be taken into account. The important role played by the magnetic domain wall dynamics in the process of the laser-induced magnetization reversal has recently been demonstrated using time-resolved magneto-optical imaging of laser-excited TbFeCo thin films (Ogasawara et al., 2009).

As shown above, in a ferrimagnet close to the compensation point both the frequency of the magnetization precession (Stanciu et al., 2006) and the domain wall velocity (Weng and Kryder, 1993; Randoshkin et al., 2003) strongly increase due to the divergence of the gyromagnetic ratio (see Sec. IV.E.4). Therefore one might expect that in such a ferrimagnetic material application of a magnetic field would instantaneously flip the magnetization. In other words, at $T_{A}$, magnetization can be regarded as a mechanical system with no inertia which can be moved by the slightest torque. However, to verify this ultrafast switching, instantaneous application of a magnetic field is required which in real experiments is not feasible. Instead, a dc magnetic field can be applied to the ferrimagnet parallel to the original magnetization direction at a temperature $T<T_{M}$. When the temperature of the ferrimagnet increases above both $T_{M}$ and $T_{A}$, its spin structure will reverse (Aeschlimann et al., 1990, 1991). Thus a femtosecond laser pulse heating of the sample might effectively act as an instantaneously applied magnetic field, allowing the investigation of the magnetization reversal speed near $T_{A}$.

The question arises: How fast is this switching process? To answer this question, hysteresis loops were measured at different time delays after the pump pulses heated the sample (Stanciu, Tsukamoto, et al., 2007). 


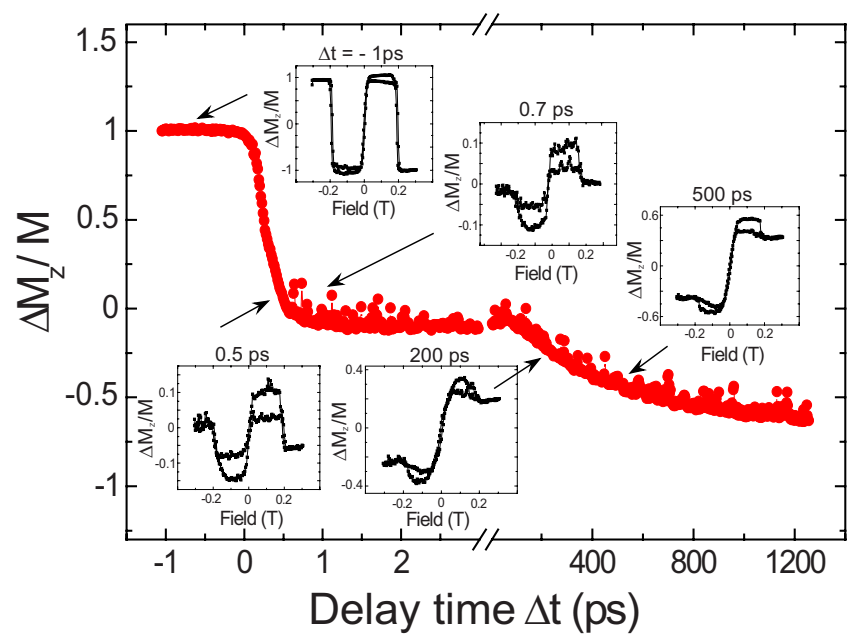

FIG. 24. (Color online) Transient magnetization reversal dynamics measured for a pump fluence of $6.29 \mathrm{~mJ} / \mathrm{cm}^{2}$. Insets show hysteresis loops measured at distinct pump-probe delays. The loops demonstrate the magnetization reversal after about 700 fs. From Stanciu, Tsukamoto, et al., 2007.

The results are shown in Fig. 24. One can observe that in this field range the measured Faraday signal changes sign after about $700 \mathrm{fs}$. The sign change reflects the change in the FeCo sublattice direction toward the applied magnetic field, as the spin temperature of the ferrimagnetic system increases over $T_{M}$ in the probed area. This observation unambiguously demonstrates that the magnetization reversal takes place on a subpicosecond time scale. Note that this reversal time is considerably faster than that found in $\mathrm{GdFeCo}$ at temperatures above the compensation points (Hohlfeld et al., 2001). On the other hand, the growth of the reversed domain to its full $100 \%$ is determined by the cooling rate and takes place at a much longer time scale ( $\sim$ nanosecond).

What are the implications of the observed subpicosecond magnetization reversal? Although it is well known that ultrafast laser excitation of itinerant ferromagnets as $\mathrm{Co}, \mathrm{Ni}$, or Fe leads to a demagnetization on the femtosecond time scale (see Sec. IV.A), little is known about how fast the magnetic moments in metals as Gd can be excited with photon energies in the visible range. This is because in Gd the optically excitable electrons of the $5 d$ - $6 s$ bands carry only $\approx 9 \%$ of the total moment, while the localized $4 f$ electrons dominate the magnetic spin moment. The localization of the magnetic moment together with the weak spin-lattice coupling that can be deduced from the small value of the magnetocrystalline anisotropy, characteristic for Gd, suggests that the transfer of the photon energy to the localized states should be a slow process of the order of 100 ps (Vaterlaus, Beutler, and Meier, 1991). In this context it was recently claimed that laser excitation of a CoGd sample resulted in an independent excitation of the Co sublattice only (Binder et al., 2006). In contrast to this, the subpicosecond magnetization reversal over the compensation points demonstrated here implies that both the Gd and FeCo sublattice magnetizations are considerably reduced on a subpicosecond time scale. In particular, an increase in the spin temperature above $T_{M}$ requires a partial demagnetization of the FeCo sublattice of $\sim 25 \%$. Consequently, the magnetization of the Gd sublattice, which below $T_{M}$ is larger than that of FeCo, must be reduced by more than $25 \%$. For $\mathrm{Gd}$ this represents a reduction in the magnetic moment far larger than that contributed by the itinerant $5 d-6 s$ electrons. A subpicosecond access to the localized $4 f$ spin moments is therefore required, thus revealing the important role played by the Gd $4 f$ electrons in this fast reversal process. Such excitation time scale is indeed allowed in Gd by the strong exchange coupling between $5 d-4 f$, responsible for its ferromagnetic order (Bovensiepen, 2006). The strength of this intra-atomic exchange is $\approx 100 \mathrm{meV}$, corresponding to $\approx 10 \mathrm{fs}$. From this it follows that indeed the localized $4 f$ spin magnetic moment in Gd can be optically excited on a time scale comparable with that observed for the itinerant ferromagnets.

\section{NONTHERMAL PHOTOMAGNETIC EFFECTS}

The laser-induced magnetization dynamics discussed above was caused by heating of electrons induced by a laser pulse that could be of any polarization. It is a dependence on the pump pulse polarization, however, that is the fingerprint of a nonthermal effect. In the following we discuss various nonthermal laser-induced effects that can lead to changes in the magnetic anisotropy, induce precessional dynamics, and even switching of the magnetization. Although all these effects do depend on the polarization of the exciting laser pulse, two different types of nonthermal effect should be distinguished. The first, so-called photomagnetic effects, do depend on the absorption of photons, resulting in an effective excitation of the system. The second group of effects are said to be optomagnetic as they do not require the absorption of photons but instead are related to, but the inverse of, magneto-optical effects such as Faraday or CottonMouton effects.

\section{A. Photomagnetic modification of magnetocrystalline anisotropy}

\section{Laser-induced precession in magnetic garnet films}

First we discuss nonthermal photomagnetic modification of the magnetocrystalline anisotropy, which was demonstrated in thin garnet films (Hansteen et al., 2005, 2006).

\section{a. Experimental observations}

Applying an external magnetic field $\mathbf{H}_{\text {ext }}$ in the plane of a magnetic garnet sample (so that $\mathbf{M}$ is in plane, $\zeta$ $=90^{\circ}$ ), see below, and pumping with linearly polarized laser pulses, optically induced precession of the magnetization $\mathbf{M}$ was observed; see Fig. 25(a). In the optical transmittivity of the sample, a sudden drop was seen which did not relax significantly within 3 ns. The amplitude and phase of the precession in Fig. 25(a) was found 

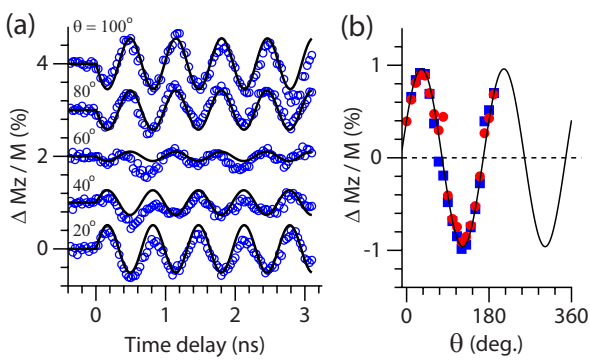

FIG. 25. (Color online) Coherent precession of the magnetization triggered by linearly polarized laser pulses. (a) Time dependence of the precession for different planes of pump polarization $\theta$, with an applied field of $\left|\mathbf{H}_{\text {ext }}\right|=350$ Oe in the plane of the sample. Circles represent measurements and solid lines simulations based on the Landau-Lifshitz equation. (b) Precessional amplitude as a function of the plane of pump polarization. Round and square symbols represent amplitudes extracted from measurements at $\pm \mathbf{H}_{\mathrm{ext}}$. The solid line is a best fit. From Hansteen et al., 2006.

to depend on the plane of polarization $\theta$ of the pump pulses as shown in Fig. 25(b). Negative values of the amplitude indicate precession of $\mathbf{M}$ with opposite phase. Maxima of the precessional amplitude were observed for every $90^{\circ}$ rotation of the polarization. From this dependence on pump polarization it is evident that the underlying effect must be nonthermal: heating would only reduce the magnitude of the magnetization and the anisotropy field, independent of the pump polarization.

It is also interesting to note that $\mathbf{M}$ always starts its precessional motion by moving normal to the film plane along the $\pm \hat{z}$ direction. This follows from the initial phase of the measured signal in Fig. 25(a), which always starts from the inflection point where $M_{z}$ is changing most rapidly. From the Landau-Lifshitz equation [Eq. (5)], it can be inferred that immediately after the photoexcitation both $\mathbf{M}$ and $\mathbf{H}_{\text {eff }}$ are in the film plane but not parallel to each other. Consequently, the observed magnetization dynamics must be due to an ultrafast change in either the magnetization $\delta \mathbf{M}$ or the anisotropy field $\delta \mathbf{H}^{a}$ or a combination of the two that effectively creates an in-plane angular displacement $\Lambda=\angle\left(\mathbf{M}, \mathbf{H}_{\text {eff }}\right)$ between $\mathbf{M}$ and $\mathbf{H}_{\text {eff. }}$ These two possibilities can be distinguished by analyzing the precession amplitude $\Lambda$ as

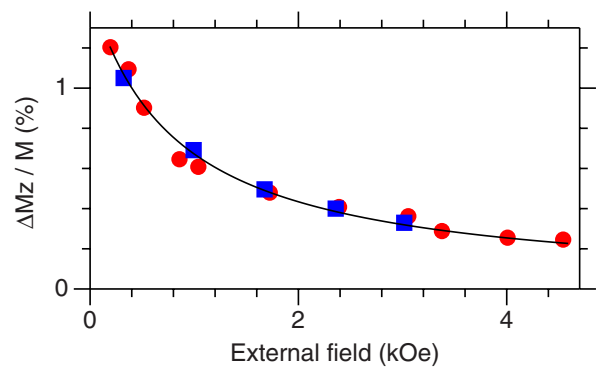

FIG. 26. (Color online) Dependence of the precessional amplitude on the applied in-plane magnetic field $\mathbf{H}_{\text {ext }}$. Round and square symbols represent amplitudes extracted from measurements at $\pm \mathbf{H}_{\text {ext }}$. From Hansteen et al., 2006.

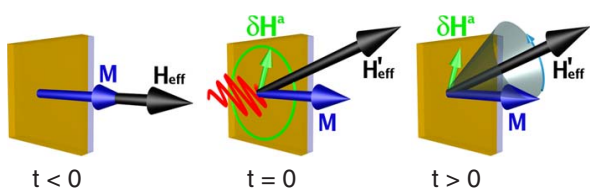

FIG. 27. (Color online) Graphical illustration of the process of photoinduced magnetic anisotropy caused by linearly polarized laser excitation and the subsequent precessional dynamics. From Hansteen et al., 2006.

function of the applied field. If triggered by an ultrafast rotation of the magnetization $\mathbf{M} \rightarrow \mathbf{M}+\delta \mathbf{M}$, the amplitude $\Lambda$ of the subsequent precession should be independent of the strength of the applied magnetic field as $\angle\left(\mathbf{M}, \mathbf{H}_{\text {eff }}\right)$ does not depend on $\mathbf{H}_{\text {ext }}$. However, if the precession is caused by a photoinduced anisotropy field $\delta \mathbf{H}^{a}$, the precession amplitude $\Lambda$ is expected to decrease with increasing applied magnetic field as

$$
\Lambda=\angle\left(\mathbf{H}_{\mathrm{eff}}, \mathbf{H}_{\mathrm{eff}}+\delta \mathbf{H}^{a}\right) \propto \frac{1}{\left|\mathbf{H}_{\mathrm{ext}}+\mathbf{H}_{a}\right|},
$$

which is valid for small-amplitude precessions, and for $\delta \mathbf{H}^{a} \perp \mathbf{H}_{\mathrm{eff}}=\mathbf{H}_{\mathrm{ext}}+\mathbf{H}_{a}$. As shown in Fig. 26 (solid line), the measurements exhibit exactly this behavior. A graphical illustration of the excitation process and the subsequent precession is shown in Fig. 27.

There appeared to be a linear relation between the precession amplitude and the pump power up to pulse energies of almost $10 \mu \mathrm{J}$, whereafter the effect saturates completely. Based on the absorption coefficient the estimated density of absorbed photons is about one per hundred unit cells in the illuminated crystal volume. Saturation effects are therefore not expected unless they are caused by the presence of a low concentration of impurities. This will be discussed in more detail below, where the microscopic basis of the photomagnetic effect is considered.

\section{b. Phenomenological model}

In this section we give a macroscopic phenomenological description of the observed photoinduced magnetic anisotropy. The model is not concerned with the microscopic mechanism of the effect, but gives some insight into its symmetry properties. ${ }^{1}$

The creation of a static magnetic field $\delta \mathbf{H}^{a}(0)$ in the garnet sample can be described as a combination of the nonlinear process of optical rectification (Shen, 1984) and a linear magnetoelectric effect (O'Dell, 1970):

\footnotetext{
${ }^{1}$ Strictly speaking, such an approach is only applicable to effects that do not involve absorption, i.e., when the result $(\delta H)$ is only present simultaneously with the stimulus $[\mathbf{E}(\omega)]$. With this restriction in mind, it is applied here to describe the symmetry properties of the effect. Time integration should actually be used on the right-hand side of Eq. (31) and below, to account for this difference. We omit this for simplicity.
} 


$$
\delta H_{i}^{a}(0)=\chi_{i j k l} E_{j}(\omega) E_{k}(\omega) M_{l}(0) .
$$

Here $\mathbf{E}(\omega)$ is the electric field component of light and $\mathbf{M}$ is the magnetization of the garnet film. The fourth-rank polar tensor $\chi_{i j k l}$ has nonzero components for crystals of any symmetry (Birss, 1966).

When the experimental geometry and the symmetry of $\chi_{i j k l}$ for the $4 \mathrm{~mm}$ point group of these samples are taken into account, only four independent nonzero components of the tensor $\chi_{i j k l}$ remain,

$$
\begin{aligned}
& A=\chi_{x x x x}=\chi_{y y y y}, \\
& B=\chi_{x y x y}=\chi_{x x y y}=\chi_{y x y x}=\chi_{y y x x}, \\
& C=\chi_{x y y x}=\chi_{y x x y}, \\
& D=\chi_{z x x z}=\chi_{z y y z},
\end{aligned}
$$

and the vector components of the photoinduced anisotropy field are then given by

$$
\begin{aligned}
\delta H_{x}^{a} \propto & E_{0}^{2} M_{s} \sin \zeta[(A+C) \cos \phi \\
& +(A-C) \cos 2 \theta \cos \phi+2 B \sin 2 \theta \sin \phi], \\
\delta H_{y}^{a} \propto & E_{0}^{2} M_{s} \sin \zeta[(A+C) \sin \phi \\
& -(A-C) \cos 2 \theta \sin \phi+2 B \sin 2 \theta \cos \phi], \\
\delta H_{z}^{a} \propto & E_{0}^{2} M_{s} D \cos \zeta .
\end{aligned}
$$

Here $\delta H_{i}^{a}$ is the photoinduced field along the $i$ direction, $i=\{x, y, z\}$ refers to the crystal axes of the sample, $\phi$ denotes the azimuthal angle between the sample $x$ axis and the projection of the magnetization vector on the film plane, and $\zeta$ is the angle between the film normal and the magnetization.

The fact that there is no amplitude offset in the curve shown in Fig. 25(b) requires that $A=-C$ so that the first term in Eqs. (33) and (34) vanishes. Furthermore, the sinusoidal shape of the curve implies that $A=B$ and leaves us with only two independent components of the tensor $\chi_{i j k l}$,

$$
\begin{aligned}
A & =\chi_{x x x x}=\chi_{y y y y}=-\chi_{x y y x}=-\chi_{y x x y} \\
& =\chi_{x y x y}=\chi_{x x y y}=\chi_{y x y x}=\chi_{y y x x}, \\
D & =\chi_{z x x z}=\chi_{z y y z} .
\end{aligned}
$$

These additional equalities indicate that the $\chi_{i j k l}$ tensor has a higher symmetry than the garnet crystal. The optically induced anisotropy field can now be written as

$$
\begin{aligned}
& \delta H_{x}^{a} \propto A E_{0}^{2} M_{s} \sin \zeta(\sin 2 \theta \sin \phi+\cos 2 \theta \cos \phi), \\
& \delta H_{y}^{a} \propto A E_{0}^{2} M_{s} \sin \zeta(\sin 2 \theta \cos \phi-\cos 2 \theta \sin \phi), \\
& \delta H_{z}^{a} \propto D E_{0}^{2} M_{s} \cos \zeta .
\end{aligned}
$$

For the in-plane field geometry $(\cos \zeta=0)$ this describes a vector of constant length and with a direction depending on the angle $\phi$ of the magnetization with respect to

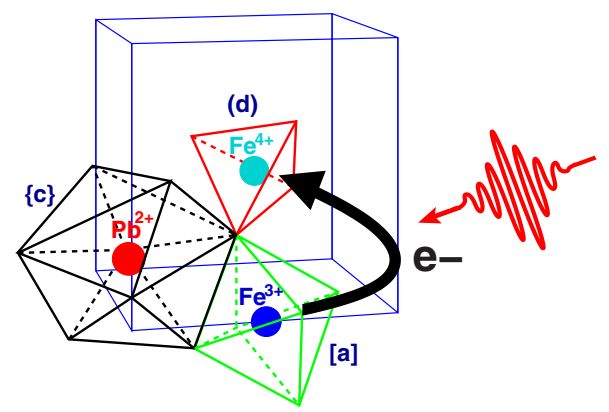

FIG. 28. (Color online) Illustration of the photoexcitation of electrons between iron ions in different crystallographic sites. A laser pulse induces electron transfer from a $\mathrm{Fe}^{3+}$ ion in the octahedral site (denoted by [a]) to a $\mathrm{Fe}^{4+}$ ion in the tetrahedral site [denoted by (d)]. The dodecahedral site with the divalent lead impurity is denoted by $\{c\}$.

the $x$ axis and the plane of polarization $\theta$ of the pump pulses.

Computer simulations based on this simple model and the numerical integration of Landau-Lifshitz equation exhibit good agreement with our experimental results, for the in-plane $\mathbf{H}_{\text {ext }}$ geometry shown in Fig. 25(a), where the results of the simulation are shown by solid lines.

\section{c. Microscopic mechanism}

Photomagnetic effects were shown to exist in garnets containing certain dopants (Teale and Temple, 1967; Dillon et al., 1969), in particular Si and Co (Chizhik et al., 1998; Stupakiewicz et al., 2001). Optically induced electron transfer between ions on nonequivalent sites in the crystal is believed to cause a change in the magnetocrystalline anisotropy due to a redistribution of ions (Alben et al., 1972). This effect is strong in crystals doped with elements that can assume different valence states and where their contribution to the anisotropy is different. However, it has also been observed in undoped garnet samples containing Pb impurities (Veselago et al., 1994), which we believe is the case here.

The linear dependence of $\delta \mathbf{H}^{a}$ for low power and the saturation at high pump power suggest that linear optical absorption by $\mathrm{Pb}$ impurities is the dominating absorption process. Divalent $\mathrm{Pb}^{2+}$ ions substitute trivalent $\mathrm{Lu}^{3+}$ ions on dodecahedral sites in the crystal and act as electron acceptors. This is a $p$-type doping which creates holes that are assumed to be located on iron ions in tetrahedral sites (Metselaar et al., 1975; Paoletti, 1978). To maintain overall charge neutrality in the crystal, some tetrahedrally coordinated trivalent iron ions change their valency to $4+$. Photoexcitation can thus induce a charge transfer between these $\mathrm{Fe}^{4+}$ ions and $\mathrm{Fe}^{3+}$ magnetic ions on octahedral sites, thus effectively "moving" the $\mathrm{Fe}^{4+}$ ions to sites with different symmetry (see Fig. 28) and thereby causing a change in the magnetic anisotropy.

The low concentration of $\mathrm{Pb}$ impurities creates a limited number of photoactive ions and the photomagnetic 


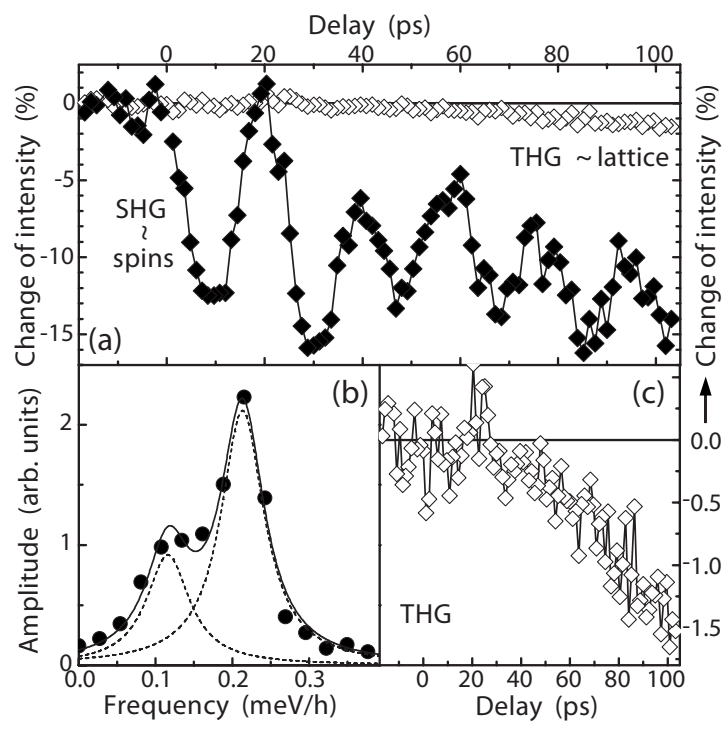

FIG. 29. Temporal behavior of second-harmonic generation (SHG) and third-harmonic generation (THG) measured in reflection from single crystal $\mathrm{NiO}(111)$ at $6 \mathrm{~K}$. (b) Fourier transform of the SHG data after subtraction of the steplike decrease at $t=0$. Dashed and straight lines: fitted spectral contributions and envelope. (c) THG signal from (a). From Duong et al., 2004.

effect can therefore be expected to saturate under intense illumination. An estimation shows that the illuminated volume of garnet film contains about $10^{12} \mathrm{~Pb}$ ions. An optical pulse of $20 \mu \mathrm{J}$ delivers $10^{14}$ photons from which about $1 \%$ is expected to be absorbed (Paoletti, 1978). This allows, in principle, for all of the photoactive ions to be excited and it is thus not surprising that saturation can occur at these pump intensities. The pumpinduced change in transmittivity is also believed to be related to the photoexcitation of impurities (Eremenko et al., 2000).

\section{Laser-induced magnetic anisotropy in antiferromagnetic $\mathrm{NiO}$}

The antiferromagnetic dielectric $\mathrm{NiO}$ has a high Néel temperature, allowing it to be used in magnetic sensors. The magnetic anisotropy in this compound is determined by dipolar and quadrupolar interactions between the $\mathrm{Ni}^{2+}$ spins. It is therefore easily modified by the shift of the $3 d$ orbital wave functions accompanying the excitation of $d-d$ transitions by an optical pump pulse. This may lead to a change in the easy direction of the singleion magnetic anisotropy from [112̄] to [111]. Duong et al. probed the magnetic changes following the photoexcitation of $\mathrm{NiO}$ by optical second-harmonic generation (SHG) (Duong et al., 2004; Satoh et al., 2006). The measurements revealed oscillations of the nonlinear optical signal with two frequencies of about 54 and $108 \mathrm{GHz}$ (see Fig. 29). It is remarkable that, in contrast to the experiments in magnetic garnets, these frequencies do not correspond to those of magnetic resonance but are close to the magnetic anisotropy energy of $0.11 \mathrm{meV}$ in- stead. The observed oscillations of the second harmonic could be tentatively explained by quantum beating, which arises from the fact that the [112] ground state and the [111] excited state interfere coherently. It was claimed, furthermore, that such a modification of the anisotropy is able to trigger an ultrafast $90^{\circ}$ switching of the antiferromagnetic vector (Fiebig et al., 2008).

Although Fiebig et al. supported their model by a number of arguments, one cannot ignore the fact that it raises some questions as well. The most important is: Why does SHG show these quantum beats but does not reveal spin oscillations at the frequency of the antiferromagnetic resonance? An instantaneous spin flip between two metastable states should trigger spin oscillations at the frequency of antiferromagnetic resonance and not at the frequency separating these two states. Unfortunately, theoretical investigations of the femtosecond response of $\mathrm{NiO}$ have not been able to address this question so far (Ney et al., 2002; Gómez-Abal et al., 2004; Lefkidis and Hübner, 2007; Lefkidis et al., 2009) and further studies of the nonlinear optical properties of this compound are required.

The experimental difficulty in this case consists in the practical impossibility of distinguishing between the dynamics of the magnetic order parameter and that of the nonmagnetic electronic contributions. Indeed, the magnetization-sensitive elements of the nonlinear optical tensor represent a not very well-understood convolution of magnetic and structural components, separation of which constitutes a major difficulty even in statics (Kirilyuk et al., 1998). Thus the oscillations in Fig. 29 might also be due to some other excitations, for example, acoustic phonons, the frequencies of which are close in the present geometry (Mazurenko and van Loosdrecht, 2007). Also, no magnetic excitations such as spin waves were observed in these experiments (Fiebig et al., 2008). Thus the study of ultrafast dynamics in purely antiferromagnetic materials remains an interesting experimental challenge for the future.

\section{Photomagnetic excitation of spin precession in (Ga,Mn)As}

Another type of nonthermal laser-induced effect, based on the absorption of photons, can be observed in ferromagnetic semiconductors, such as GaMnAs. For instance, Wang et al. reported on spin precession in GaMnAs induced by 100 fs laser pulses (Wang, Ren, et al., 2007). These results were interpreted in terms of a model based on the temperature dependence of the magnetic anisotropy. In particular, the temperature rise that follows the absorption of the laser pulse was claimed to be responsible for a rapid change in magnetic anisotropy and consequently for the change in the equilibrium orientation of the magnetization. Such a change on a time scale much faster than the period of magnetic resonance in GaMnAs led to the excitation of a homogeneous spin precession. The problem, however, is that such a mechanism would require either a modulation of the magnetic shape anisotropy due to an ultrafast demagnetization of GaMnAs, as usually observed in met- 

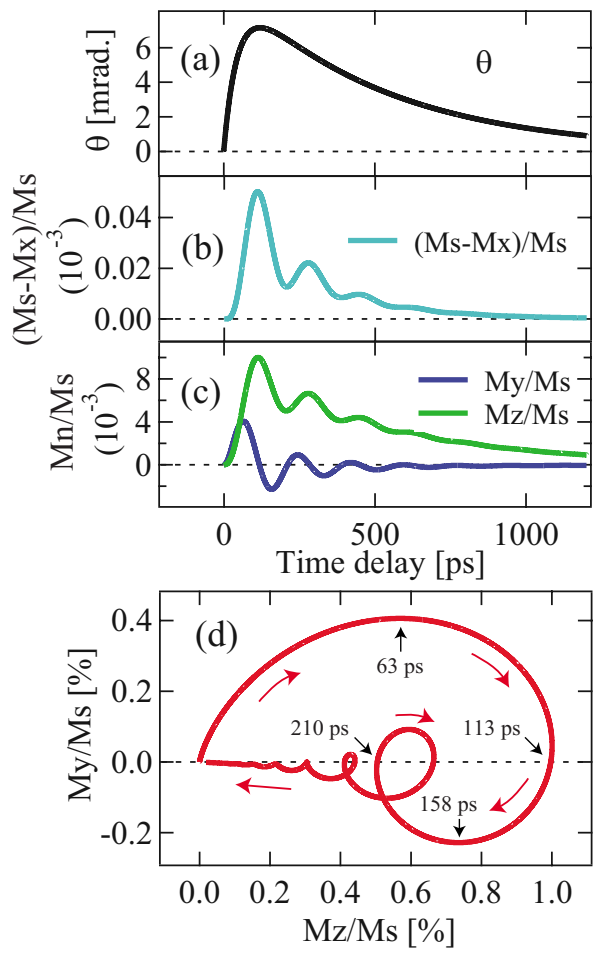

FIG. 30. (Color online) Laser-induced magnetic anisotropy and subsequent spin precession in $\mathrm{Ga}_{0.8} \mathrm{Mn}_{0.2} \mathrm{As}$. (a) Temporal profiles of the angle of tilt of the effective magnetic field $H_{\text {eff }}$ experienced by the spins. The time dependence originates from transient photoinduced magnetic anisotropy in the material, (b) temporal evolution of the $x$ component of magnetization, and (c) $y$ and $z$ as function of time. All profiles are extracted on the basis of experimental curves. (d) Precessional motion of magnetization in $M_{y}-M_{z}$ parameter space (Hashimoto et al., 2008).

als (van Kampen et al., 2002), or a modulation of the magnetocrystalline anisotropy via an increase in the lattice temperature; see Kimel, Kirilyuk, et al. (2004) and Bigot et al. (2005). Both effects normally require pump fluences of about $1 \mathrm{~mJ} / \mathrm{cm}^{2}$, while the phenomenon reported by Wang et al. was triggered by a substantially lower pump fluence of $0.2 \mu \mathrm{J} / \mathrm{cm}^{2}$ (photon energy of $1.5 \mathrm{eV})$. The effect was found to be insensitive to the polarization of the pump. Soon a similar surprisingly effective laser excitation of a homogeneous spin precession in GaMnAs was reported in experiments with a photon energy of $1.569 \mathrm{eV}$ and a pump fluence of $3.4 \mu \mathrm{J} / \mathrm{cm}^{2}$ (Hashimoto et al., 2008); see Fig. 30. The latter was again much too small for a substantial demagnetization, required to excite precession via a change in shape anisotropy.

To solve this problem, trajectories of the laser-induced spin motion were analyzed. Simulations based on the Landau-Lifshitz-Gilbert equation showed that one can describe the oscillations if one assumes that the laser pulse introduces an impulsive change in the effective magnetic field due to a change in the magnetic anisotropy. The amplitude of the impulse was estimated to be about $0.2 \mathrm{~T}$. The impulse was characterized by a rise time of $50 \mathrm{ps}$ and a decay time of 500 ps. It was noted that such a pulse shape suggests the presence of a postphotocarrier process. Hashimoto et al. speculated that such photoinduced anisotropy change may be due to a change in the number of holes near the Fermi level. The after effect is associated with the time required for the laser-generated holes to cool down via emission of acoustic phonons.

Chovan and Perakis analyzed the problem of laser control of magnetization in magnetic semiconductors using density matrix equations of motion in a meanfield and single-band approximation (Chovan et al., 2006; Chovan and Perakis, 2008). They showed that in (III,Mn)V semiconductors one may expect a modification of the magnetic anisotropy on the time scale of the optical pulse duration, so that a laser pulse acts on the Mn spin as an effective magnetic field. Unfortunately, qualitative estimates of the strength of the effective magnetic field and pump fluences required for this effect have not been made. Recently Wang et al. reported that an excitation of GaMnAs with a pump fluence of $10 \mu \mathrm{J} / \mathrm{cm}^{2}$ and photon energy of $3.1 \mathrm{eV}$ allowed to detect a magnetization rotation during the action of the laser pulse (Wang et al., 2009). A comparison of the amplitude of the transient magneto-optical signal and static magneto-optical measurements allowed the estimation that during the action of the laser pulse (200 fs) the magnetization in GaMnAs deviated over $0.5^{\circ}$ from equilibrium. Taking the gyromagnetic ratio for GaMnAs from Liu et al. (2003) and Wang, Ren, et al. (2007), one may estimate that such a deviation of the magnetization from equilibrium can be observed if the laser pulse acts as an effective magnetic field with an amplitude of $0.1 \mathrm{~T}$. This is comparable with the value reported by Hashimoto et al. (2008). However, Wang et al. (2009) also stated that the pumping of GaMnAs with a photon energy of $3.1 \mathrm{eV}$ was crucial for the observation of the femtosecond magnetization rotation. Unfortunately, the calculations of Chovan and Perakis were performed in a single-band approximation and thus did not allow for the analysis of the spectral dependence of the photoinduced magnetic anisotropy or the revelation of the origin of the differences between the findings reported by these three experimental groups (Wang, Ren, et al., 2007; Hashimoto et al., 2008; Wang et al., 2009).

\section{B. Light-enhanced magnetization in (III,Mn)As semiconductors}

Surprising and intriguing observations were obtained when a (III,Mn)As semiconductor sample was irradiated by low-power continuous radiation. In (In,Mn)As such an excitation with unpolarized light (fluence of the laser equivalent to about 50 photons $/ \mathrm{cm}^{2}$ per $100 \mathrm{fs}$ ) led to an enhancement of the magnetization (Koshihara et al., 1997). For a long time, time-resolved experiments aiming to reveal the relevant time scales of such an enhancement remained a challenge. Only recently an ultrafast enhancement of magnetic order in GaMnAs as a result of excitation with 120 fs linearly polarized pulses with a photon energy of $3.1 \mathrm{eV}$ and peak fluences $\sim 10 \mu \mathrm{J} / \mathrm{cm}^{2}$ was reported by Wang, Cotoros, et al. 


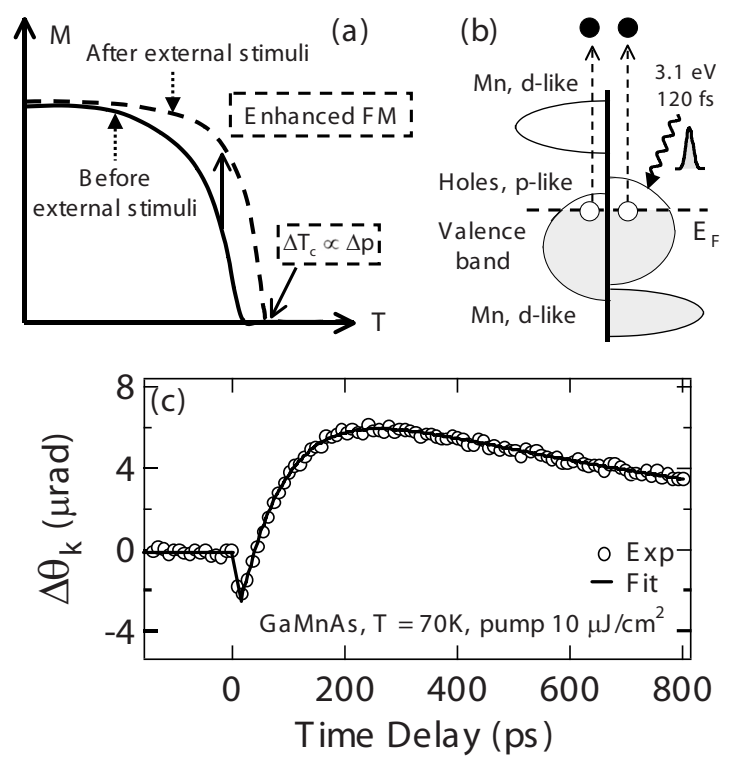

FIG. 31. (Color online) Laser-induced magnetism in GaMnAs: (a) Illustration of hole-density-tuning effects via external stimuli in III-V FMSs seen in the static experiments (Koshihara et al., 1997). FM: ferromagnetism. $4 p$ is hole density change. (b) Schematic diagram of the spin-dependent density of states in GaMnAs. Femtosecond pump pulses create a transient population of holes in the valence band. (c) Timeresolved magneto-optical Kerr effect dynamics at $70 \mathrm{~K}$ and under $1.0 \mathrm{~T}$ field. Transient enhancement of magnetization, with $\sim 100$ ps rise time, is clearly seen after initial fast demagnetization. From Wang, Cotoros, et al., 2007.

(2007); see Fig. 31. No effect of the polarization of light on this optical control of magnetic order was found. However, both the initial subpicosecond demagnetization and a distinct magnetization rise on a 100 ps time scale were observed. The observed ultrafast enhancement of magnetization was attributed to laser-induced photoinjection of holes and subsequent enhancement of collective ordering of $\mathrm{Mn}$ spins, leading to a transient increase in the Curie temperature over $0.5-1.1 \mathrm{~K}$. According to the Zener model (Dietl et al., 2000) this value is in reasonable agreement with the ratio between background and photoexcited holes.

An excitation of $(\mathrm{Ga}, \mathrm{Mn})$ As with circularly polarized cw light was claimed to lead to a large helicitydependent change in magnetization, reaching up to $15 \%$ of the saturation magnetization (Oiwa et al., 2002); see Fig. 32. Note that (III,Mn)As semiconductors possessing ferromagnetic order have a relatively high concentration of holes of the order of $10^{20} \mathrm{~cm}^{3}$. The laser-induced changes by low-power excitation $\left(4 \times 10^{5}\right.$ photons $/ \mathrm{cm}^{2}$ per $100 \mathrm{fs}$ ) in combination with the fast hole-spin relaxation ( $\sim 10 \mathrm{fs})$ is surprising. These experiments have immediately raised questions about the mechanisms and relevant time scales of such an extremely efficient optical control of magnetism. Two attempts to investigate these questions with the help of time-resolved magnetooptical measurements have been done (Kimel, Astakhov, et al., 2004; Mitsumori et al., 2004). Although different interpretations of these experimental results were

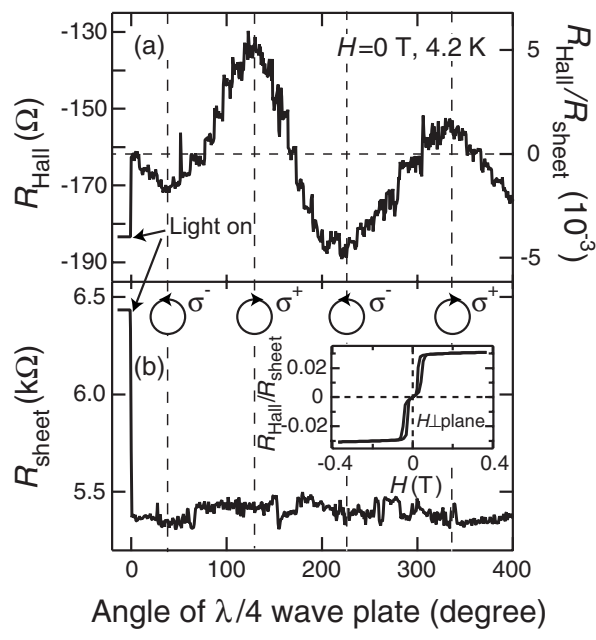

FIG. 32. Changes in Hall resistance and sheet resistance at 4.2 K for a GaMnAs/GaAs sample under cw light irradiation, the polarization state of which is controlled by a quarter waveplate. The inset shows the magnetization curve extracted from the anomalous Hall effect measurements, indicating in-plane magnetic anisotropy of the current sample. From Oiwa et al., 2002.

suggested, none of these dynamic measurements was able to show that $100 \mathrm{fs}$ laser pulses induce large values of photoinduced magnetization, as reported for $\mathrm{cw}$ excitation (Oiwa et al., 2002). The mechanism that would allow efficient control of magnetization in GaMnAs is still an open question that is a subject of intense research interest (Aoyama et al., 2008; Astakhov et al., 2009).

\section{NONTHERMAL OPTOMAGNETIC EFFECTS}

\section{A. Inverse magneto-optical excitation of magnetization dynamics: Theory}

We now discuss nonthermal laser-induced effects that do not depend on the absorption of photons. In Sec. II it was shown both phenomenologically and microscopically that circularly polarized light can directly influence spins in magnetically ordered materials on a femtosecond time scale. The suggested mechanism for laser control of spins does not require annihilation of photons and the light-induced spin flip does not require the loss of the angular momentum of the photons. Instead, the photons stimulate an efficient angular momentum transfer from spins (by generating magnons) via orbits to the lattice.

\section{Formal theory of inverse optomagnetic effects}

A formal mathematical theory is considered here for a transparent magnetic dielectric (Gridnev, 2008). ${ }^{2}$ Taking into account the small wave vectors $k \approx 0$ of light, only

\footnotetext{
${ }^{2}$ Following the original reference (Gridnev, 2008), the Gaussian CGS system of units is used in this section.
} 
spin-wave modes with $k=0$ are considered, that is, the temporary behavior of $\mathbf{M}$ is homogeneous across the sample.

The deviation of $\mathbf{M}(t)$ from equilibrium is given by the components $M_{x}$ and $M_{y}$, the $z$ axis directed along the equilibrium magnetic moment $\mathbf{M}(0)$. For the following consideration, it is convenient to introduce the canonical variables $\{b\}$ through the linearized Holstein-Primakoff transformation:

$$
b=(2 \gamma M)^{-1 / 2}\left(M_{x}+i M_{y}\right),
$$

where $M=|\mathbf{M}|$ is the magnitude of the sublattice magnetization and $\gamma$ is the gyromagnetic ratio. In the harmonic approximation, the Hamiltonian $H_{0}$ of the spin system is a quadratic function of the variables $\left\{b, b^{*}\right\}$.

The electric field of a pump pulse exciting the spin system can be represented in the form $\mathbf{E}(t)=\operatorname{Re} \mathcal{E}(t) e^{i \omega t}$, where $\omega$ is the central frequency of the pulse and $\mathcal{E}(t)$ is the time-dependent amplitude.

Phenomenologically, the interaction between magnons (or other low-energy collective excitations) and the pump pulse in a transparent medium is given by (Landau and Lifshitz, 1984)

$$
V=-\frac{\delta \epsilon_{i j}}{16 \pi} \mathcal{E}_{i}^{*}(t) \mathcal{E}_{k}(t)
$$

where $\delta \epsilon_{i j}$ is the modulation of the optical dielectric permittivity by magnons. $V$ can be expressed as a power series in $b$ and $b^{*}$. When considering spin-wave excitations, one should take into account only terms in $\delta \epsilon_{i j}$ that are linear in $b$ and $b^{*}$. For a transparent medium, the tensor $\delta \epsilon_{i j}$ is Hermitian: $\delta \epsilon_{i j}=\delta \epsilon_{j i}^{*}$.

In the impulsive limit, when the optical pulse width $\tau \ll \Omega^{-1}$, where $\Omega$ is the frequency of the induced spin wave, one may use the formal representation

$$
\mathcal{E}_{i}^{*}(t) \mathcal{E}_{j}(t)=\frac{4 \pi I_{0}}{n c} e_{i}^{*} e_{j} \delta(t),
$$

where $n$ is the refractive index, $c$ is the speed of light, $I_{0}$ is the integrated pulse intensity, and $e_{i}$ is a component of the unit vector defining the polarization of light. The delta function $\delta(t)$ accounts for the impulsive character $\left(\tau \ll \Omega^{-1}\right)$ of the pump light. It is convenient to decompose the product $e_{i}^{*} e_{j}$ into its symmetric and antisymmetric parts,

$$
e_{i}^{*} e_{j}=S_{i j}+\frac{i}{2} a_{i j k} h_{k},
$$

where $S_{i j}=\frac{1}{2}\left(e_{i}^{*} e_{j}+e_{j}^{*} e_{i}\right), a_{i j k}$ is the antisymmetric unit tensor, and $h_{k}$ is a component of the vector $\mathbf{h}=i\left(\mathbf{e} \times \mathbf{e}^{*}\right)$. $|\mathbf{h}|$ is a measure of the light helicity and varies in the range from -1 to 1 .

Under the action of the light pulse, excluding absorption, $b$ varies with time according to the Hamilton equation (Zakharov and Kuznetsov, 1984),

$$
\frac{d b}{d t}=-i \frac{\partial}{\partial b^{*}}\left(H_{0}+V\right) .
$$

The advantage of the classical approach used here is that there is no need to specify exactly the optical transitions caused by the photoexcitation. The optical properties of a medium relevant for the generation of coherent excitations are contained in the modulation of the optical dielectric permittivity. This is a consequence of the fact that all optical transitions are virtual for a transparent medium. When optical absorption is significant, this approach does not work and a microscopic consideration is necessary (Stevens et al., 2002).

Equations (41) and (44) are rather general and can be applied to pump pulses of arbitrary duration including cw light as a limiting case, provided that the electric field entering the interaction potential [Eq. (41)] is the total electric field, i.e., is the sum of the incident $\mathcal{E}^{I}$ and scattered $\mathcal{E}^{S}$ electric fields. Despite the smallness of the scattering field in comparison with the incident one, $\mathcal{E}^{S}$ in Eq. (41) may not always be neglected. When considering stimulated Raman scattering (RS) of $\mathrm{cw}$ light, it is the term containing the product $\left(\mathcal{E}_{i}^{I}\right)^{*} \mathcal{E}_{j}^{S}$ that is responsible for the scattering (and for spin-wave generation) because $\mathcal{E}^{I}$ and $\mathcal{E}^{S}$ oscillate at different frequencies. The case of impulsive stimulated RS (ISRS) (Yan et al., 1985) is quite a different situation. In this case, the spectral width of the excitation pulses exceeds the spin-wave frequency. For this reason, in the impulsive stimulated scattering process, higher-frequency photons from a pump pulse are coherently scattered into lower-frequency photons within the pump pulse bandwidth and propagated in the same direction but with a slightly smaller wave vector magnitude. Thus, ISRS is a forward-scattering process which is stimulated because the Stokes frequency is contained within the bandwidth of the incoming pulse. The pump pulse is asymmetrically redshifted through ISRS when it leaves the sample. Since the intensity of the scattered light is small compared to the incident one, the scattered electric field can be neglected in the interaction potential.

Equation (44) can be applied to any instant of time, including times within the pump pulse, $t<\tau$. However, experimentally, light-induced spin polarization can be reliably measured only at times after the pump pulse, $t>\tau$. For this reason, it makes sense to calculate the induced magnetization at those times. In that case, it is allowed to take the limit of zero pump pulse duration, $\tau \rightarrow 0$.

Integrating Eq. (44) over the pulse width and accounting for Eq. (42), we obtain $b$ just after the photoexcitation $\left(t=0^{+}\right)$,

$$
b\left(0^{+}\right)=i \frac{I_{0}}{4 n c} \sum_{i j} \frac{\partial \delta \epsilon_{i j}}{\partial b^{*}} e_{i}^{*} e_{j} .
$$

Thus, in general, immediately after the photoexcitation the spins are rotated away from the equilibrium direction. This is simply a consequence of the fact that the electron spins straightforwardly participate in the optical transitions induced by the light pulse.

Equations (44) and (45) are applicable to all coherent collective excitations, which are characterized by non- 
zero values of the canonical variables. Note that this is not the case for coherent two-magnon excitations (Zhao et al., 2004). For magnons, $\delta \epsilon_{i j}$ depends, in general, on both $\operatorname{Re} b \sim M_{x}$ and $\operatorname{Im} b \sim M_{y}$. Consequently, both real and imaginary parts of $b\left(0^{+}\right)$are finite at $t=0^{+}$, i.e., each spin acquires a finite rotation during a pump pulse.

In order to study the temporal evolution of the spin system with $N$ sublattices, it is convenient to transform $b_{r}$, where $r=1, \ldots, N$ is a site index, to the normal coordinates $Q_{\alpha}$,

$$
Q_{\alpha}=\sum_{r}\left(u_{r \alpha} b_{r}+v_{r \alpha} b_{r}^{*}\right),
$$

where $\alpha=1, \ldots, N$ is a mode index and $u_{r \alpha}$ and $v_{r \alpha}$ are matrices, satisfying the following equations that guarantee that the transformation [Eq. (46)] is canonical:

$$
\begin{aligned}
& \sum_{r}\left(u_{r \alpha} u_{r \alpha^{\prime}}^{*}-v_{r \alpha} v_{r \alpha^{\prime}}^{*}\right)=\delta_{\alpha \alpha^{\prime}}, \\
& \sum_{r}\left(u_{r \alpha} v_{r \alpha^{\prime}}^{*}-v_{r \alpha} u_{r \alpha^{\prime}}^{*}\right)=0 .
\end{aligned}
$$

After the transformation to normal coordinates, the Hamiltonian of the spin system has the simple form

$$
H_{0}=\sum_{\alpha} \Omega_{\alpha} Q_{\alpha} Q_{\alpha}^{*}
$$

where $\Omega_{\alpha}$ is the frequency of the spin-wave mode $\alpha$. Being canonical variables, the normal coordinates also obey the Hamilton equations

$$
\frac{d Q_{\alpha}}{d t}+i \Omega_{\alpha} Q_{\alpha}=-i \frac{\partial V}{\partial Q_{\alpha}^{*}} .
$$

The modulation of the dielectric permittivity by the spin waves is given by

$$
\delta \epsilon_{i j}=\sum_{\alpha}\left(P_{i j}^{\alpha} Q_{\alpha}+R_{i j}^{\alpha} Q_{\alpha}^{*}\right),
$$

where

$$
P_{i j}^{\alpha}=\partial \delta \epsilon_{i j} / \partial Q_{\alpha}, \quad R_{i j}^{\alpha}=\partial \delta \epsilon_{i j} / \partial Q_{\alpha}^{*}
$$

are scattering matrices for the mode $\alpha$. Solving Eq. (49) for $Q_{\alpha}$ and taking into account Eqs. (41), (42), and (50), we obtain

$$
Q_{\alpha}=i \frac{I_{0}}{4 n c} \sum_{i j} R_{i j}^{\alpha} e_{i} e_{j}^{*} e^{-i \Omega_{\alpha} t} .
$$

These laser-induced magnetic excitations, in turn, lead to changes in the dielectric permittivity of the medium. For these, using Eqs. (50) and (52) we obtain

$$
\delta \epsilon_{i j}^{\text {ind }}=i \frac{I_{0}}{4 n c} \sum_{n m \alpha}\left(P_{i j}^{\alpha} R_{n m}^{\alpha} e^{-i \Omega_{\alpha} t}-P_{n m}^{\alpha} R_{i j}^{\alpha} e^{i \Omega_{\alpha} t}\right) e_{n} e_{m}^{*} .
$$

Thus, Eq. (53) explicitly shows the oscillatory response in the pump-probe experiments. The particular phase of the measured oscillations will depend on the properties of the given medium, such as the magnetic symmetry as well as the magnetocrystalline anisotropy that determine the properties of the scattering matrices $P_{i j}^{\alpha}$ and $R_{i j}^{\alpha}$. The latter contain all information about the dependence of the spin-wave generation on the light polarization, crystal orientation, and magnetic structure. In order to calculate these matrices, one needs to know the modulation of the dielectric permittivity $\delta \epsilon_{i j}$ induced by the deviation of the spin system from equilibrium. $\delta \epsilon_{i j}$ can be expanded in powers of the sublattice magnetizations (Cottam and Lockwood, 1986). To reveal the distinctive features of the magnon generation by an optical pulse in more detail, we perform the explicit calculations of the light-induced spin precession for a simple cubic ferromagnet and a rutile-type structure antiferromagnet.

\section{Example I: Cubic ferromagnet}

For a simple cubic ferromagnet $(N=1)$ the magnetization-dependent part of $\epsilon_{i j}$ is given by (Cottam and Lockwood, 1986)

$$
\epsilon_{i j}=i K \sum_{k} e_{i j k} M_{k}+\sum_{k l} G_{i j k l} M_{k} M_{l}
$$

where the first and second terms on the right-hand side describe the Faraday and Cotton-Mouton (Voigt) effects, respectively.

For simplicity, let the external magnetic field $\mathbf{H}$ and the equilibrium magnetization $\mathbf{M}_{0}$ point along the $z$ axis. Then the change in the permittivity as a function of the spin-wave amplitudes $M_{x}(t)$ and $M_{y}(t)$ is

$$
\delta \epsilon_{i j}=\sum_{k=x, y}\left(i K e_{i j k} M_{k}+2 G_{i j k z} M_{0} M_{k}\right) .
$$

Our ferromagnet possesses only one spin-wave eigenmode with the frequency $\Omega=\gamma H$ and the normal coordinate

$$
Q=\left(2 \gamma M_{0}\right)^{-1 / 2}\left(M_{x}+i M_{y}\right) .
$$

Using Eqs. (55) and (56) we obtain the scattering matrices

$$
\begin{aligned}
& P_{i j}=\sqrt{\frac{\gamma M_{0}}{2}}\left[K\left(i e_{i j x}+e_{i j y}\right)+2 M_{0}\left(G_{i j x z}-i G_{i j y z}\right)\right], \\
& R_{i j}=\sqrt{\frac{\gamma M_{0}}{2}}\left[K\left(i e_{i j x}-e_{i j y}\right)+2 M_{0}\left(G_{i j x z}+i G_{i j y z}\right)\right],
\end{aligned}
$$

Then, using Eqs. (52) and (56) the light-induced dynamic components of the magnetization are derived:

$$
\begin{aligned}
& M_{x}(t)=-c_{1} \cos \Omega t+c_{2} \sin \Omega t, \\
& M_{y}(t)=c_{2} \cos \Omega t+c_{1} \sin \Omega t,
\end{aligned}
$$

where

$$
\begin{aligned}
& c_{1}=\beta\left(K h_{y}+G M_{0} S_{y z}\right), \\
& c_{2}=\beta\left(K h_{x}+G M_{0} S_{x z}\right),
\end{aligned}
$$

with $G=G_{x z x z}=G_{y z y z}$ and $\beta=\gamma M_{0} I_{0} / 4 n c$. Although in a cubic ferromagnet the tensor $G_{i j k l}$ has three independent components, only one of them relates to the one- 
magnon RS when $\mathbf{M}_{0} \|[001]$ (Cottam and Lockwood, 1986). According to Eqs. (58) and (59), the initial change in magnetization $\Delta \mathbf{M}=\left\{M_{x}\left(0^{+}\right), M_{y}\left(0^{+}\right)\right\}=\left\{-c_{1}, c_{2}\right\}$ consists of two contributions, $\Delta \mathbf{M}=\Delta \mathbf{M}_{K}+\Delta \mathbf{M}_{G}$, proportional to the Faraday and Cotton-Mouton magnetooptical constants, respectively. $\Delta \mathbf{M}_{K}$ vanishes for linearly polarized light $(\mathbf{h}=\mathbf{0})$, while $\Delta \mathbf{M}_{G}$ can be nonzero for any light polarization.

Note that the magneto-optical constants $K$ and $G$ define the Faraday rotation and magnetic linear birefringence, respectively, and can be determined by measurements of these effects. In magnetic dielectrics, $K M_{0} \sim 0.001$ and $G M_{0} / K$ usually lies in the range of $0.1-1$. Because the magnetic linear birefringence is relatively strong in magnetically ordered media (Ferré and Gehring, 1984), it may happen that linearly and circularly polarized light can induce spin waves of comparable amplitude (see Sec. VI.D.2 below).

\section{Example II: Two-sublattice antiferromagnet}

Here we consider a two-sublattice $(N=2)$ antiferromagnet with a tetragonal symmetry $D_{4 h}$, with spins oriented along the fourfold $z$ axis. The spin-wave spectrum of such an antiferromagnet consists of two branches that are degenerate in zero magnetic field, with a frequency

$$
\Omega=\gamma \sqrt{H_{A}\left(H_{A}+2 H_{E}\right)},
$$

where $H_{E}$ is the exchange field and $H_{A}$ is the anisotropy field. In this case, the coefficients $u_{r \alpha}$ and $v_{r \alpha}$ defining the normal coordinates are given by (Fleury and Loudon, 1968; Kittel, 1987);

$$
\begin{array}{ll}
u_{11}=u, & v_{21}=v, \\
u_{22}=u, & v_{21}=v_{11}=0,
\end{array}
$$

where

$$
u, v=\sqrt{\frac{\gamma\left(H_{E}+H_{A}\right) \pm \Omega}{2 \Omega}} .
$$

From these, the normal coordinates for the two modes are

$$
Q_{1}=u b_{1}+V b_{2}^{*}, \quad Q_{2}=u b_{2}+v b_{1}^{*},
$$

where $b_{1,2}$ are defined by Eq. (40).

To describe the light-induced spin dynamics of the antiferromagnet in more detail, it is convenient to introduce the ferromagnetic and antiferromagnetic vectors $\mathbf{M}=\mathbf{M}_{1}+\mathbf{M}_{2}$ and $\mathbf{L}=\mathbf{M}_{1}-\mathbf{M}_{2}$. The components $M_{x}$ and $M_{y}$ characterize mainly the relative orientation of the sublattice magnetizations $\mathbf{M}_{1}$ and $\mathbf{M}_{2}$, while $L_{x}$ and $L_{y}$ describe their deviations away from the $z$ axis. To calculate these components, we start with the calculation of the normal coordinates $Q_{1,2}(t)$ from Eq. (52).

The interaction of the nonequilibrium spin system of the antiferromagnet with light is given by (Cottam, 1975; Cottam and Lockwood, 1986)

$$
\begin{aligned}
V= & K_{+}\left(M_{x} h_{x}+M_{y} h_{y}\right)+K_{-}\left(L_{x} h_{y}+L_{y} h_{x}\right) \\
& +G_{+}\left(L_{x} S_{x z}+L_{y} S_{y z}\right)+G_{-}\left(M_{x} S_{x z}+M_{y} S_{y z}\right),
\end{aligned}
$$

where $K_{ \pm}$and $G_{ \pm}$are magneto-optical constants. Next, using Eqs. (40), (47), and (52), one obtains

$$
\begin{aligned}
& M_{x}=-m_{1} \cos \Omega t+l_{2}(u-v)^{2} \sin \Omega t, \\
& M_{y}=m_{2} \cos \Omega t+l_{1}(u-v)^{2} \sin \Omega t, \\
& L_{x}=-l_{1} \cos \Omega t+m_{2}(u+v)^{2} \sin \Omega t, \\
& L_{y}=l_{2} \cos \Omega t+m_{1}(u+v)^{2} \sin \Omega t,
\end{aligned}
$$

where

$$
\begin{aligned}
& m_{1}=\beta\left(K_{-} h_{x}+G_{+} S_{y z}\right), \\
& m_{2}=\beta\left(K_{-} h_{y}+G_{+} S_{x z}\right), \\
& l_{1}=\beta\left(K_{+} h_{y}+G_{-} S_{y z}\right), \\
& l_{2}=\beta\left(K_{+} h_{x}+G_{-} S_{x z}\right),
\end{aligned}
$$

Equations (65) show that, as in a ferromagnet, the spins in the antiferromagnet are rotated during the light pulse, and this rotation is independent of $H_{E}$ and $H_{A}$. Moreover, the parameters $m_{1}$ and $m_{2}$ determine the angle between the sublattice magnetizations just after the pump pulse. These parameters depend on the polarization of the pump pulse as well as on the magneto-optical constants $K$ and $G$. The initial noncollinearity leads to an enhancement of the spin rotation from the $z$ axis in the subsequent motion.

\section{B. Excitation of precessional magnetization dynamics with circularly polarized light}

\section{Optical excitation of magnetic precession in garnets}

Garnets show a minimum of absorption at the excitation wavelength of $800 \mathrm{~nm}$. The key factor, however, in distinguishing the nonthermal effects described below is their dependence on the pump pulse polarization. Thus, left- and right-handed circularly polarized laser pulses incident along the $z$ direction were used to excite a magnetic garnet film exposed to an in-plane applied magnetic field $\mathbf{H}_{\text {ext }}$. Precession of $\mathbf{M}$ with opposite phase was triggered by pulses of helicity $\sigma^{+}$and $\sigma^{-}$; see Fig. 33. The initial phase of the signal reveals that $\mathbf{M}$ initially moves along the $\pm z$ direction and therefore both $\mathbf{M}$ and $\mathbf{H}_{\text {eff }}$ are parallel to the film plane immediately after the photoexcitation.

These experimental observations can be understood if during the presence of the laser pulse a strong magnetic field along the $\mathbf{k}$ vector of light is created. Such an axial magnetic field $\mathbf{H}^{F}$ can be generated by intense circularly polarized light through what is known as the inverse Faraday effect (Pitaevskii, 1961; van der Ziel et al., 1965; Pershan et al., 1966); see Sec. II.C. These optically generated field pulses are much stronger than both the an- 


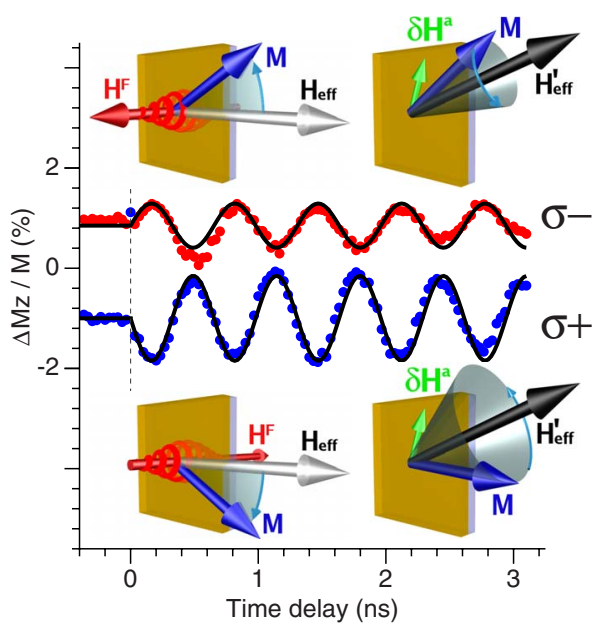

FIG. 33. (Color online) Precession following excitation with circularly polarized light. The two helicities $\sigma^{+}$and $\sigma^{-}$give rise to precession with an opposite phase and a different amplitude. During the $100 \mathrm{fs}$ presence of the laser pulse the magnetization precesses in the dominating axial magnetic field $\mathbf{H}_{F}$ created by the circularly pump pulse. Subsequent precession takes place in the effective magnetic field $\mathbf{H}_{\text {eff }}^{\prime}$, possibly modified by the photomagnetic effects, see Sec. V.A. From Hansteen et al., 2005, 2006.

isotropy field $\mathbf{H}_{a}$ and the applied field $\mathbf{H}_{\mathrm{ext}}$ and therefore completely dominate during the $\Delta t=100$ fs presence of the laser pulse. The magnetization will respond by precessing in the plane of the film (normal to $\mathbf{H}^{F}$ ) to a new in-plane orientation. After the pulse is gone, the magnetization will precess in the effective in-plane field $\mathbf{H}_{\text {eff }}^{\prime}$ $=\mathbf{H}_{\mathrm{ext}}+\mathbf{H}_{a}+\delta \mathbf{H}^{a}$, as shown in Fig. 33, where $\delta \mathbf{H}^{a}$ is the optically induced change in the anisotropy field.

The strength of the photoinduced field $\mathbf{H}^{F}$ can be estimated from the precession amplitude $\Lambda$ :

$$
H^{F} \approx \omega / \gamma \approx \Lambda / \gamma \Delta t_{\text {pulse }},
$$

where $\omega$ is the precession frequency, $\gamma$ is the gyromagnetic ratio, and $\Delta t_{\text {pulse }}$ is the duration of the optical pulse. We find that laser pulses of energy $20 \mu \mathrm{J}$ create transient magnetic field pulses of about $0.6 \mathrm{~T}$ in the garnet films.

\section{Optical excitation of antiferromagnetic resonance in $\mathrm{DyFeO}_{3}$}

The optomagnetic inverse Faraday effect can be used to excite magnetization dynamics in a situation when any other method is difficult or impossible to apply. In this section we describe optical excitation of antiferromagnetic resonance modes in $\mathrm{DyFeO}_{3}$, in the hundreds of $\mathrm{GHz}$ frequency range.

Details of the structure of this antiferromagnet have been given by Wijn (1994). Because of the Dzyaloshinskii-Moriya interaction, the antiferromagnetically coupled $\mathrm{Fe}$ spins are slightly canted, giving rise to a spontaneous magnetization $M_{s} \sim 8 \mathrm{G}$. Despite the small magnetization, this material exhibits a giant Faraday rotation of about $3000^{\circ} \mathrm{cm}^{-1}$ owing to its strong

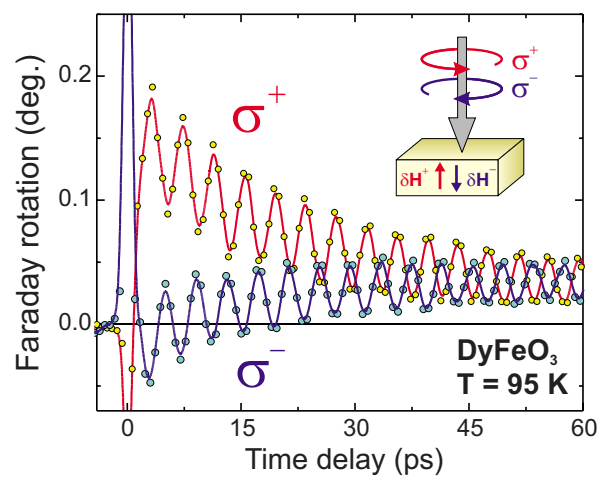

FIG. 34. (Color online) Magnetic excitations in $\mathrm{DyFeO}_{3}$ probed by the magneto-optical Faraday effect. Two processes can be distinguished: (1) instantaneous changes of the Faraday effect due to the photoexcitation of $\mathrm{Fe}$ ions and relaxation back to the high spin ground state $S=5 / 2$ and (2) oscillations of the Fe spins around their equilibrium direction with an approximately 5 ps period. The circularly polarized pump pulses of opposite helicities excite oscillations of opposite phase. Inset shows the geometry of the experiment. Vectors $\delta \mathbf{H}^{+}$and $\delta \mathbf{H}^{-}$represent the effective magnetic fields induced by righthanded $\sigma^{+}$and left-handed $\sigma^{+}$circularly polarized pump pulses, respectively. From Kimel et al., 2005.

spin-orbit interaction (Zvezdin and Kotov, 1997). Such weak ferromagnets represent an intriguing combination of the static properties of a ferromagnet with the dynamics of an antiferromagnet.

For the detection of the optically induced magnetization the direct magneto-optical Faraday effect was used, which was possible due to the presence of a weak ferromagnetic moment. Figure 34 shows the temporal evolution of the Faraday rotation in a $z$-cut $\mathrm{DyFeO}_{3}$ sample for two circularly polarized pump pulses of opposite helicities. On the scale of $60 \mathrm{ps}$ one can distinguish two different processes that start after excitation with a pump pulse. At zero time delay instantaneous changes in the Faraday rotation are observed that result from the excitation of virtual and real transitions in the $\mathrm{Fe}^{3+}$ ions from the high-spin ground state $S=5 / 2$. The instantaneous changes of the Faraday rotation are followed by oscillations with a frequency of about $200 \mathrm{GHz}$ that can be assigned to oscillations of the magnetization. It is seen from Fig. 34 that the helicity of the pump light controls the sign of the photoinduced magnetization. This observation unambiguously indicates that the coupling between spins and photons in $\mathrm{DyFeO}_{3}$ is direct because the phase of the spin oscillations is given by the sign of the helicity of the exciting photon.

The amplitude of the oscillations corresponds to a photoinduced change in the magnetization $\Delta M$ $\sim 0.06 M_{s}$, where $M_{s}$ is the saturation magnetization.

From Fig. 34 one can distinguish not only oscillations but also an exponential decay of the equilibrium level on a time scale of about $30 \mathrm{ps}$. This can be explained by a photoinduced change in the equilibrium orientation of the magnetization and a subsequent decay of this equilibrium orientation to the initial state. 


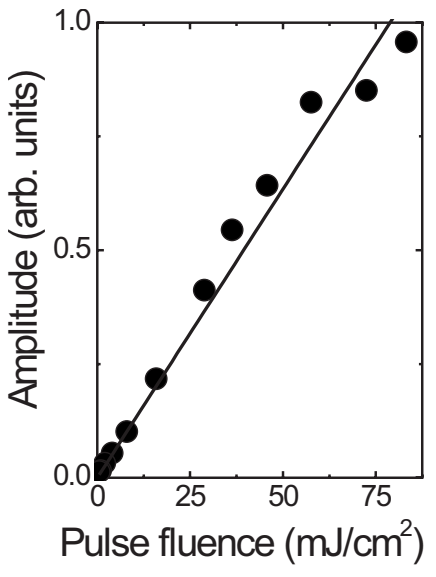

FIG. 35. The amplitude of the spin oscillations as a function of pump fluence. From Kimel et al., 2005.

Although in principle the effect of optically induced magnetization does not require the absorption of photons, the laser control of the spontaneous magnetization and the excitation of coherent spin oscillations is equivalent to photoexcitation of magnons and thus requires some energy. Figure 35 shows the amplitude of the photoexcited spin oscillations as a function of the pump intensity. The linearity of this dependence is in perfect agreement with the expression for the inverse Faraday effect [Eq. (16)]. Note that extrapolation of the intensity dependence shows that the photoinduced effect on the magnetization would reach the saturation value of $M_{s}$ at a pump fluence of about $500 \mathrm{~mJ} / \mathrm{cm}^{2}$. The effect of such a $100 \mathrm{fs}$ laser pulse on the magnetic system would be equivalent to the application of a magnetic field pulse of about $5 \mathrm{~T}$. According to our measurements, the absorption in $\mathrm{DyFeO}_{3}$ in the near infrared spectral range is on the order of $100-200 \mathrm{~cm}^{-1}$. Given this low value of the absorption, a photoexcitation of $500 \mathrm{~mJ} / \mathrm{cm}^{2}$ is still below the damage threshold of $\mathrm{DyFeO}_{3}$ and thus quite feasible, provided the sample is of high optical quality.

\section{Optical excitation of precession in GdFeCo}

In the two examples above, the inverse Faraday effect was shown to act as an effective magnetic field on the spins in oxidic materials. The observed effects were nonthermal and any additional laser-induced thermal effects were small in these transparent crystals. But what about metals? Most magnetic materials of practical interest are ferromagnetic metals. Would a circularly polarized laser pulse also act as a magnetic field pulse in this case?

Many attempts to detect an effect of the light helicity on ferromagnetic metals such as $\mathrm{Ni}$ and other transition metals remained unsuccessful (Ju, Vertikov, et al., 1998; Wilks et al., 2004; Kruglyak, Hicken, et al., 2005). Heatdriven effects of light were found to dominate in all these experiments. In order to diminish the unavoidable effects due to laser-induced heating, we used the approach of turning the sample into a multidomain state, in this way minimizing the usually dominating heat-

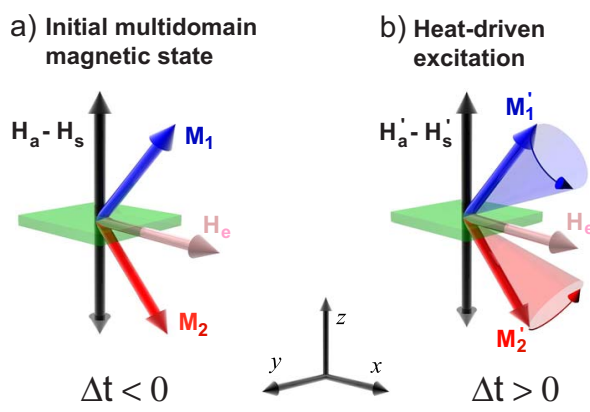

FIG. 36. (Color online) Suppression of heat-driven oscillations of the net magnetization in a multidomain sample: (a) At $\Delta t<0$, the magnetizations $\mathbf{M}_{1}$ and $\mathbf{M}_{2}$ of oppositely magnetized domains are tilted due to the balance among magnetocrystalline anisotropy field $\mathbf{H}_{a}$, shape anisotropy field $\mathbf{H}_{s}$, and external field $\mathbf{H}_{e}$. (b) After photoexcitation, $\Delta t>0$, heating induces out-of-phase oscillations of the $z$ components of $\mathbf{M}_{1}$ and $\mathbf{M}_{2}$.

driven effects of light on the magnetization, as explained below.

The material chosen for this study was the ferrimagnetic alloy GdFeCo, discussed in Sec. IV.G. The GdFeCo 20-nm-thick layer is amorphous and is characterized by a strong perpendicular anisotropy and a Curie temperature of about $500 \mathrm{~K}$.

Initially, the sample was in a single-domain state. The application of an out-of-plane opposite field with the simultaneous presence of an in-plane external magnetic field results in the nucleation of a large number of small domains (Wu, 1997; Kisielewski et al., 2003), with sizes much smaller than the probe area. Because of the coercivity, these magnetic domains are sufficiently stable in time and thus suitable for stroboscopic pump-probe experiments. The balance among magnetocrystalline anisotropy field $\mathbf{H}_{a}$, shape anisotropy field $\mathbf{H}_{s}$, and external field $\mathbf{H}_{e}$ creates an initial state for these experiments such that the magnetization in each of the domains will be tilted [see Fig. 36(a)]. Laser excitation of such a sample induces locally a change in the magnetocrystalline and shape anisotropy due to heating. This leads to a change in the equilibrium orientation for the magnetization and triggers a precession of the magnetic moments of the domains. These precessions proceed in such a way that the $z$ components of the magnetization in oppositely oriented domains [like $\mathbf{M}_{1}$ and $\mathbf{M}_{2}$ in Fig. 36(b)] always oscillate out of phase. Since the probe beam is averaged over a large number of such oppositely oriented domains, the heat-driven effect of light on the magnetization is effectively averaged out in the Faraday signal by achieving $\Sigma \delta \mathbf{M}_{1}^{i}+\Sigma \delta \mathbf{M}_{2}^{j} \approx 0$, where the sums are taken over all domains within the probed area. Experimentally, the optimum cancellation is achieved by adjusting the external magnetic field $\mathbf{H}_{e}$. On the other hand, an optically induced effective magnetic field will apply a torque on all the various magnetic moments in the same direction and consequently lead to a nonzero change in $M_{z}$. 


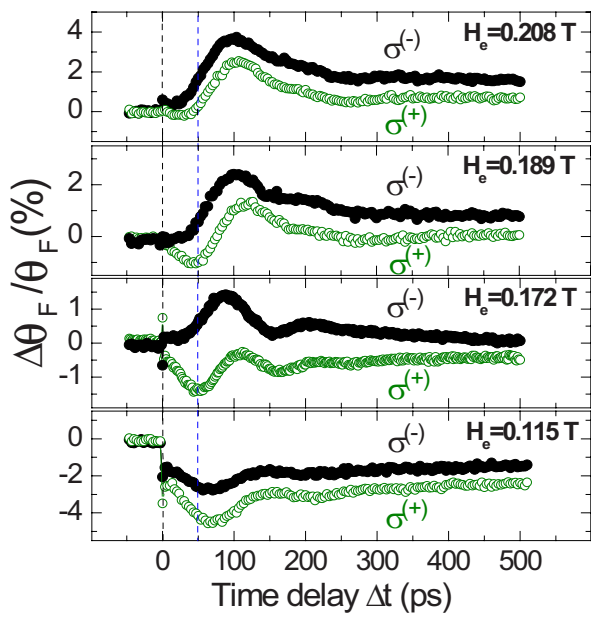

FIG. 37. (Color online) Precession of the magnetization excited by circularly polarized pump pulses in GdFeCo, probed via the magneto-optical Faraday effect. For certain values of the external field $\mathbf{H}_{e}$, the two helicities $\sigma^{+}$and $\sigma^{-}$give rise to precession with different phase. The lines are guides to the eye.

The results of these time-resolved measurements of the magneto-optical Faraday effect for right-handed $\sigma^{+}$ and left-handed $\sigma^{-}$circularly polarized pump pulses at different $\mathbf{H}_{e}$ are shown in Fig. 37. The variation in the magneto-optical signal $\Delta \theta_{F}$, representing the oscillatory behavior of $\mathbf{M}_{z}$, was plotted relative to the total Faraday rotation of the sample $\theta_{F}$. On the scale of 500 ps one can distinguish two different processes. At zero time delay, instantaneous changes in the Faraday rotation are observed that result partly from ultrafast demagnetization and partly from changes in the magnetocrystalline anisotropy (Vomir et al., 2005). These instantaneous changes of the Faraday rotation are followed by oscillations with a frequency of about $7 \mathrm{GHz}$, which can be ascribed to precession of the magnetization (Stanciu et al., 2006). The observed strong damping can be understood as a result of the averaging over the many domains. Figure 37 shows that the difference between the magnetooptical Faraday signal for right- and left-handed excitation strongly depends on the external applied field $\mathbf{H}_{e}$. At $\mathbf{H}_{e}=0.172 \mathrm{~T}$ the $\sigma^{+}$and $\sigma^{-}$pump pulses excite precession of opposite phases. Because the phase of the spin precession is given by the polarization of the exciting photons, this observation provides a first indication of an optomagnetic effect in the metallic ferrimagnet GdFeCo.

Equation (16) shows that the strength of the effective magnetic field $\mathbf{H}_{F}$ depends on the laser fluence. Although in these experiments a relatively high laser fluence for a metallic magnetic material $\left(2 \mathrm{~mJ} / \mathrm{cm}^{2}\right)$ was used, this is still low compared with the experiments on nonabsorbing dielectrics [e.g., $60 \mathrm{~mJ} / \mathrm{cm}^{2}$ for garnets (Hansteen et al., 2006)] where a strong optomagnetic effect was observed. Therefore the usual experimental conditions for metallic magnets of low laser fluence in addition to a strong heat-driven effect require a special

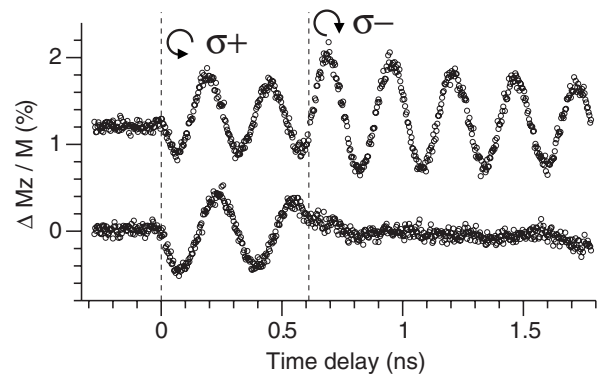

FIG. 38. Double-pump experiment in magnetic garnet with circularly polarized laser pulses of opposite helicity. Shown is how amplification and complete stopping of the magnetization precession can be achieved depending on the phase of the precession when the second laser pulse arrives. The time delay between the two pump pulses is fixed at approximately $0.6 \mathrm{~ns}$, and the precession frequency is controlled by varying the external field. From Hansteen et al., 2006.

technique for the detection of ultrafast optomagnetic effects, as shown here. The observation of the inverse Faraday effect in a metallic magnet together with the previously discussed observation of this effect in orthoferrites and garnets indicate that this mechanism does not rely on specific material properties but is a general phenomenon [see also Hertel (2006)]. Its strength, as well as the experimental possibility of observing it, depend on the material parameters. On the one hand, it is the value of the magneto-optical response that determines the nonthermal interaction. On the other hand, there are competing thermal effects that are usually dominating in metals. To make a classification, the role of the bandwidth may be invoked: narrowband materials, such as insulators, oxides, and to some extent also $f$ metals can be excited much more selectively than the broadband transition metals. Similarly, the former exhibit more of a helicity dependence than the latter. This explains, for example, the absence of helicity-dependent effects in the experiments on $\mathrm{Ni}$ (Longa et al., 2007).

\section{All-optical control and switching}

\section{Double-pump coherent control of magnetic precession}

The nonthermal effects of light on the magnetization allow for ultrafast coherent control of spin precession. Such control can be achieved using multiple laser pulses in rapid succession.

In Fig. 38 the results of such coherent control experiments are shown for a magnetic garnet. Initially, for $t<0$ the spins are aligned along the total effective magnetic field $\mathbf{H}_{\text {eff }}$ that is of the order of $0.05 \mathrm{~T}$. A pump pulse of helicity $\sigma^{+}$arriving at $t=0$ acts as a strong pulse of magnetic field $H^{F} \gg H_{\text {eff }}$ and thus it triggers precession of the magnetization, as explained in the previous sections. A second pump pulse of helicity $\sigma^{-}$arriving after an odd number of half-precessional periods rotates the magnetization further away from $\mathbf{H}_{\text {eff }}$, causing the subsequent precession to have almost twice the amplitude (upper graph). If, however, this second pump pulse 
(a)

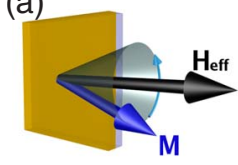

100 fs $<\mathrm{t}<\mathrm{t}_{2}$

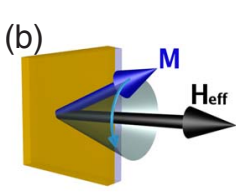

$100 \mathrm{fs}<\mathrm{t}<\mathrm{t}_{2}$

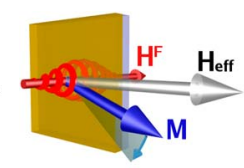

$t_{2}<t<t_{2}+100 f s$

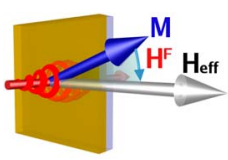

$t_{2}<t<t_{2}+100 f s$

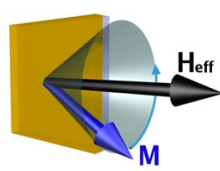

$t>t_{2}+100$ fs

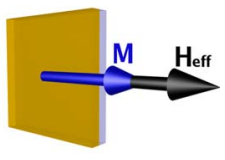

$t>t_{2}+100 f s$

FIG. 39. (Color online) Illustration of the double-pump experiment for circularly polarized pump pulses of opposite helicity arriving at an (a) odd number of half precessional periods and (b) an integer number of full precessional periods. The magnetization is either rotated further away from the effective field direction causing subsequent precession to take place with almost twice the original amplitude or the magnetization is rotated back into the effective field direction and no further precession takes place. From Hansteen et al., 2006.

arrives after an integer number of full periods, the magnetization is rotated back into its original equilibrium orientation along $\mathbf{H}_{\text {eff }}$ and no further precession takes place (lower graph). Figure 39 gives a pictorial illustration of these two situations. Similarly, circularly polarized light can control the precession of antiferromagnetic spins in the terahertz domain (see Fig. 40).

These experiments demonstrate that femtosecond optical pulses can be used to directly and coherently control spin motions. Depending on the phase of the precession when the second pulse arrives, energy is transferred either from the laser pulse to the magnetic

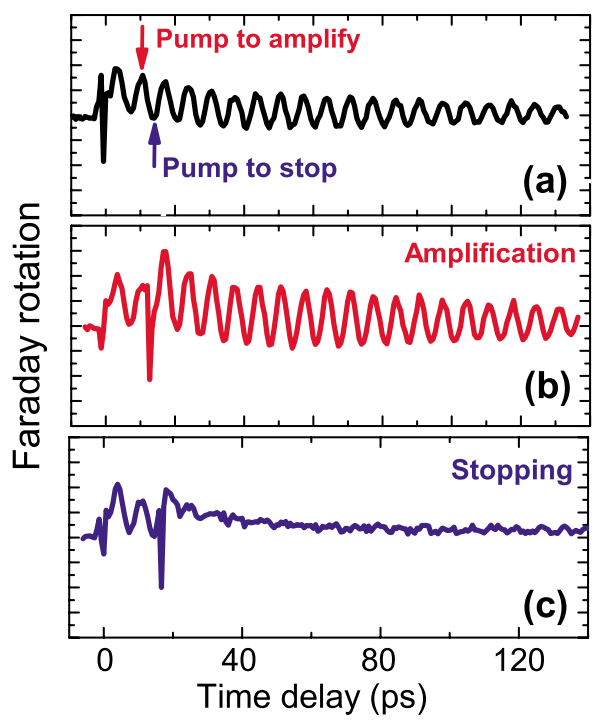

FIG. 40. (Color online) Coherent control of spins in $\mathrm{DyFeO}_{3}$ with two circularly polarized laser pulses. (a) Precession triggered by the first laser pulse, (b) amplification of spin precession by the second laser pulse that comes after an even number of full periods, and (c) stopping of the spin oscillations by the second pump that comes after an odd number of half periods.

system (amplification of the precession) or from the magnetic excitation to the optical pulse (stopping of the precession). In view of the low intrinsic damping in the orthoferrites and garnets and therefore the long lifetime of their magnetic excitations, it is remarkable how ultrashort laser pulses can completely and instantaneously stop the long-period coherent precession of spins. This process of transferring the energy back into the optical pulse can also be viewed as coherent laser cooling of magnons.

The complex spin oscillations in orthoferrites triggered by a train of laser pulses was recently studied theoretically using nonlinear Landau-Lifshitz-Gilbert equations. It was demonstrated that such a periodical excitation of spins results in various patterns of spin oscillations, which depend on the intensity and periodicity of the laser pulses (Perroni and Liebsch, 2006).

It should be pointed out that the present doublepump experiments, which demonstrate control of the magnetization in ferrimagnetic garnets and antiferromagnetic orthoferrites, are considerably different from those previously reported in diamagnetic and paramagnetic materials. During the past two decades many publications have been devoted to the photoexcitation of a nonequilibrium spin polarization in direct-band-gap semiconductors through the phenomenon of optical orientation (Meier and Zakharchenya, 1984; Awschalom et al., 1987; Žutić et al., 2004). In these materials, absorption of circularly polarized photons may lead to a nonequilibrium population of spin-polarized electrons and holes in the conduction and valence bands, respectively. In paramagnetic semiconductors these spin-polarized carriers can cause partial alignment of the moments of magnetic ions due to an $s p$ - $d$ exchange interaction and thereby also affect their precession in a magnetic field (Furdyna and Kossut, 1988). Using this phenomenon of optical orientation, Akimoto et al. (1998) demonstrated control of the precession of $\mathrm{Mn}^{2+}$ moments in $\mathrm{CdTe} / \mathrm{Cd}_{1-x} \mathrm{Mn}_{x}$ Te quantum wells.

A nonabsorptive mechanism for manipulation of spins in $\mathrm{Zn}_{1-x} \mathrm{Cd}_{x} \mathrm{Se}$ quantum well structures was reported by Gupta et al. (2001), who used below-band-gap optical pulses to control the spin precession of photoexcited electrons in the conduction band via the optical Stark effect. However, these experiments were performed on paramagnetic materials, where coupling between the spins of magnetic ions is small and the spins oscillate independently. Therefore, in a double-pump experiment with paramagnets, the first and second laser pulses can simply excite different spins so that the integrated signal will show either amplification or quenching of the oscillations. However, the amplification and quenching of the oscillations in such an experiment would only mean that the spins excited by the first or second pump pulses oscillate in phase or out of phase, respectively. In magnetically ordered materials, discussed in this review, spins are strongly coupled by the exchange interaction and spin excitations are delocalized. Therefore, in contrast to paramagnets, laser control of spins in magnetically or- 


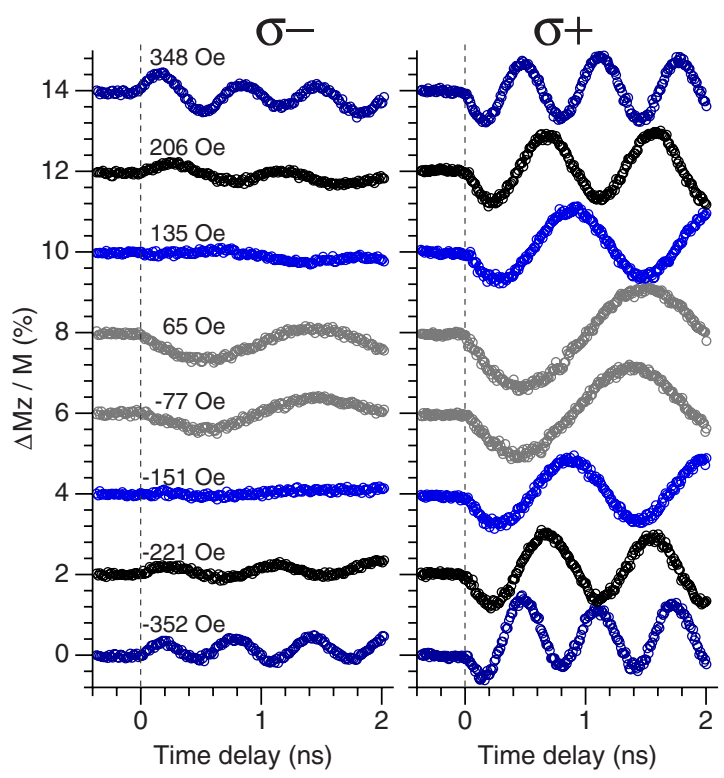

FIG. 41. (Color online) Precession of the magnetization triggered by left- and right-handed circularly polarized laser pulses at different values of the in-plane applied magnetic field. For the $\sigma^{-}$helicity, at an applied field of $\sim \pm 150 \mathrm{Oe}$, no precession is observed due to a perfect balance of the two photomagnetic effects $\delta \mathbf{H}^{a}$ and $\mathbf{H}^{F}$. From Hansteen et al., 2005.

dered materials indeed means control of the collective motion of spins.

\section{Femtosecond switching in magnetic garnets}

A proper combination of the inverse Faraday effect and the photoinduced anisotropy (Sec. V.A) allows for an interesting demonstration of photomagnetic switching on a femtosecond time scale (Hansteen et al., 2005). When the laser pulse is circularly polarized, the direction of $\delta \mathbf{H}^{a}$ depends only on the initial angle $\phi$ of the magnetization with respect to the crystal axes. Therefore, it can be tuned by rotating the sample with respect to the applied field. Alternatively, since the initial equilibrium of $\mathbf{M}$ is along $\mathbf{H}_{\text {eff }}$, which is determined by the balance between the magnetocrystalline anisotropy field $\mathbf{H}_{a}$ and the externally applied field $\mathbf{H}_{\text {ext }}$, it can also be tuned simply by varying the strength of the applied field.

In Fig. 41 the coherent precession of the magnetization following excitation with pulses of helicity $\sigma^{-}$and $\sigma^{+}$is shown for different values of $\mathbf{H}_{\text {ext }}$. The amplitude of precession is consistently larger in the case of $\sigma^{+}$, as during $0<t<100 \mathrm{fs}$, M precesses away from the new equilibrium created by $\delta \mathbf{H}^{a}$. For pulses of helicity $\sigma^{-}$, this precession is toward the new equilibrium, leading to smaller precessional amplitude in the time after the pulse. With an applied field of $\left|\mathbf{H}_{\text {ext }}\right| \approx 150$ Oe, no precession is triggered owing to a perfect balance of two effects: the in-plane precession of the magnetization during the $100 \mathrm{fs}$ magnetic field pulse $\delta \mathbf{H}^{F}$ brings the magnetization exactly to its new equilibrium orientation created by the simultaneously optically modified anisotropy field. It remains stable in this orientation until the aniso-

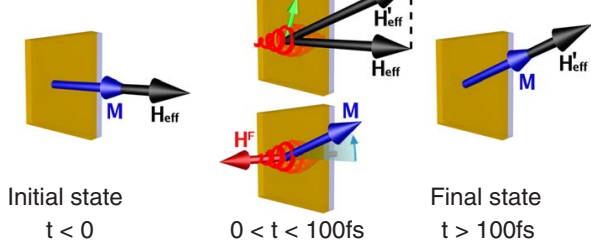

FIG. 42. (Color online) Illustration of the switching process. Initially at $t<0$ the magnetization is along $\mathbf{H}_{\text {eff }}$. During the presence of the laser pulse $0<t<100$ fs photoinduced modification of the anisotropy fields leads to a new long-lived equilibrium along $\mathbf{H}_{\mathrm{eff}}^{\prime}$. Simultaneously, the strong optomagnetically generated field $\mathbf{H}^{F}$ causes the magnetization to precess into the new state. After $t>100$ fs the optical pulse is gone and the approximately $0.6^{\circ}$ switching of $\mathbf{M}$ is complete. From Hansteen et al., 2005.

tropy field relaxes back to its original state, i.e., for several nanoseconds. An illustration of this switching process is shown in Fig. 42.

Note also that for the $\sigma^{-}$helicity at weak applied fields the precession has an opposite phase compared to the precession in stronger applied fields and that this phase is the same as for the precession triggered by the $\sigma^{+}$pulses. At weak fields the direction of the photoinduced $\delta \mathbf{H}^{a}$ is such that the precession of $\mathbf{M}$ by $\mathbf{H}^{F}$ during the optical pulse is not sufficient to bring it into the direction of $\mathbf{H}_{\text {eff }}^{\prime}$. At stronger fields, however, $\delta \mathbf{H}^{a}$ is in a different direction producing an $\mathbf{H}_{\text {eff }}^{\prime}$ that is less inclined with respect to the original effective field. During the presence of $\mathbf{H}^{F}$ the magnetization now precesses past the direction of $\mathbf{H}_{\text {eff }}^{\prime}$ and therefore with the opposite phase in the time directly after the laser pulse.

\section{Inertia-driven switching in antiferromagnets}

The dynamics of spins in a ferromagnet is described by the LLG equation that does not contain inertial terms such as acceleration. Therefore, a switching between two minima requires that the system is dragged over the potential barrier. However, if an inertial motion of the magnetic moments, similar to that of a finite-mass object was possible, one could "kick" the system so that the following motion will bring the system over the barrier and into the new equilibrium (see Fig. 43). Such a mechanism would allow the use of pulses much shorter than the precessional periods.

The inertial motion can actually be realized in antiferromagnets (Kimel et al., 2009). The dynamics of an antiferromagnet with two sublattices $\mathbf{M}_{1}$ and $\mathbf{M}_{2}$ is described in terms of the motion of the antiferromagnetic unit vector $\mathbf{I}=\left(\mathbf{M}_{1}-\mathbf{M}_{2}\right) /\left|\mathbf{M}_{1}-\mathbf{M}_{2}\right|$. In angular variables, the equation of motion can be written as (Kimel et al., 2009)

$$
\frac{d^{2} \varphi}{d t^{2}}+2 \Gamma \frac{d \varphi}{d t}+\omega_{0}^{2} \frac{d w(\varphi)}{d \varphi}-\frac{\gamma^{2} H_{D}}{\sin \theta_{0}} H(t) \cos \varphi=0 .
$$

Here the presence of the acceleration $d^{2} \varphi / d t^{2}$ describes the appearance of inertia, similar to that in the Newton equation of motion for unit mass; the second term pre- 

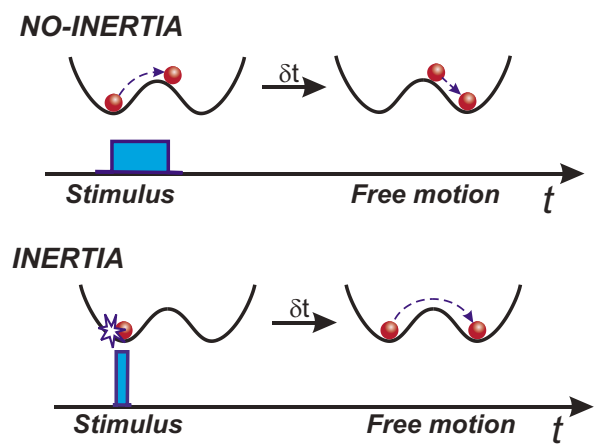

FIG. 43. (Color online) Noninertial and inertial scenarios to transfer a point mass over a potential. The noninertial mechanism (above) requires a continuous driving force that pulls the mass over the potential barrier. A similar scenario is realized in magnetization reversal via precessional motion in ferromagnets. In contrast, in the inertial mechanism (below), during the action of the driving force the coordinate of the particle is hardly changed, but the particle acquires enough momentum to overcome the barrier afterwards. From Kimel et al., 2009.

sents a viscous force with damping coefficient $\Gamma$; terms $d w(\varphi) / d \varphi$ and $H_{D}$ represent the restoring and driving force, respectively; $w(\varphi)$ is a dimensionless function proportional to the magnetic anisotropy energy; $\omega_{0}$ is the frequency of the lower antiferromagnetic mode.

A short magnetic field pulse should trigger an inertial spin reorientation between two magnetic phases. To observe this, however, the pulse must be shorter than the characteristic time of the magnetic eigenmodes, a few picoseconds in the case of an antiferromagnet. Such short pulses can be provided only by the inverse Faraday effect.

To observe such inertial motion of spins in $\mathrm{HoFeO}_{3}$, pump-probe experiments were performed with $100 \mathrm{fs}$ circularly polarized laser pulses. The pump-induced spin dynamics was monitored by detecting the $M_{z}$ component of the magnetization via the magneto-optical Faraday effect in the probe pulse, measured as a function of delay between the pump and probe pulses. The results of such time-resolved measurements were in excellent agreement with the results of simulations and thus demonstrated the inertial motion of antiferromagnetically coupled spins (Kimel et al., 2009).

\section{All-optical magnetization reversal}

In Sec. IV.G we showed how laser-induced heating of GdFeCo in an external magnetic field, induced a subsequent ultrafast reversal (switching) of the magnetization. As is obvious from the previous sections, circularly polarized femtosecond laser pulses act as equally short magnetic field pulses via the inverse Faraday effect. This naturally leads to the question: Can such optically induced field pulses be used to completely reverse the magnetization of a magnetic domain?

The experiments were performed by placing a sample of the GdFeCo magnetic alloy under a polarizing microscope, where domains with magnetization "up" and "down" could be observed as white and black regions,

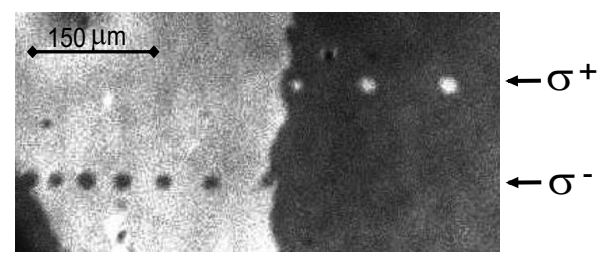

FIG. 44. The effect of single $40 \mathrm{fs}$ circular polarized laser pulses on the magnetic domains in $\mathrm{Gd}_{22} \mathrm{Fe}_{74.6} \mathrm{Co}_{3.4}$. The domain pattern was obtained by sweeping at high-speed $(\sim 50 \mathrm{~mm} / \mathrm{s})$ circularly polarized beams across the surface so that every single laser pulse landed at a different spot. The laser fluence was about $2.9 \mathrm{~mJ} / \mathrm{cm}^{2}$. The small size variation in the written domains is caused by the pulse-to-pulse fluctuation of the laser intensity. From Stanciu, Hansteen, et al., 2007.

respectively. To excite the material, amplified pulses from a Ti:sapphire laser were used at a wavelength of $\lambda=800 \mathrm{~nm}$, repetition rate of $1 \mathrm{kHz}$, and a pulse width of $40 \mathrm{fs}$. The laser pulses were incident normal to the sample surface, so that an effective optically generated magnetic field would be directed along the magnetization, similar to a conventional recording scheme.

In order to unambiguously determine whether excitation by a single $40 \mathrm{fs}$ laser pulse is sufficient to reverse the magnetization, the laser beam was swept at high speed across the sample so that each pulse landed at a different spot; see Fig. 44. One can see that each of the $\sigma^{+}$pulses reversed the magnetization in the black domain but did not affect the magnetization of the white domain. The opposite situation is observed when the sample is exposed to $\sigma^{-}$pulses. Thus, during the presence of a single $40 \mathrm{fs}$ laser pulse, information about the photons' angular momentum is transferred to the magnetic medium and subsequently recording occurs. These experiments unambiguously demonstrate that all-optical magnetization reversal can be achieved by single 40 fs circularly polarized laser pulses without the aid of an external magnetic field.

As a simple illustration of optomagnetic recording it is shown in Fig. 45 how optically written bits can be overlapped and made much smaller than the beam waist by

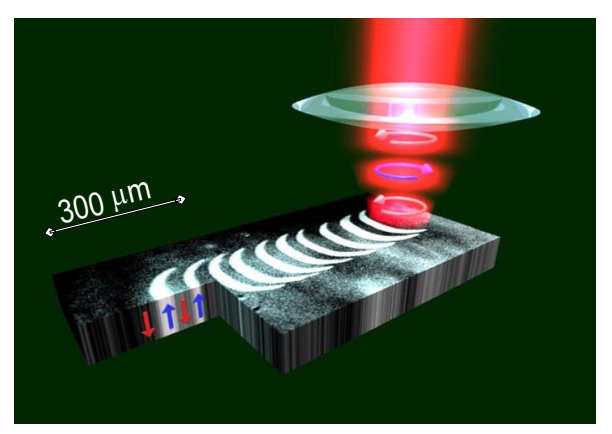

FIG. 45. (Color online) Demonstration of compact all-optical recording of magnetic bits. This was achieved by scanning a circularly polarized laser beam across the sample and simultaneously modulating the polarization of the beam between left and right circular. From Stanciu, Hansteen, et al., 2007. 


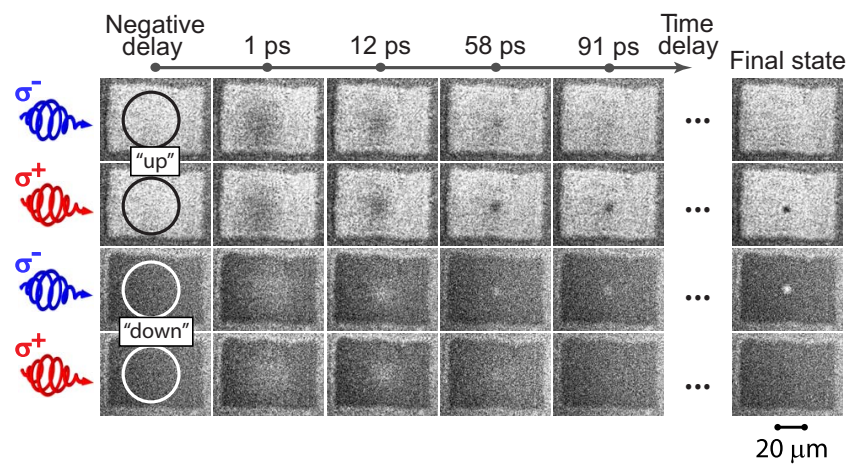

FIG. 46. (Color online) The magnetization evolution in $\mathrm{Gd}_{24} \mathrm{Fe}_{66.5} \mathrm{Co}_{9.5}$ after the excitation with right- $\left(\sigma^{+}\right)$and lefthanded $\left(\sigma^{+}\right)$circularly polarized pulses at room temperature. The domain is initially magnetized "up" (white domain) and "down" (black domain). The last column shows the final state of the domains after a few seconds. The circles show areas actually affected by pump pulses. From Vahaplar et al., 2009.

modulating the polarization between $\sigma^{+}$and $\sigma^{-}$as the laser beam is swept across the sample. High-density recording may be achieved in principle by employing especially designed near-field antenna structures (Challener et al., 2003) such as those currently being developed for heat-assisted magnetic recording (HAMR).

\section{Reversal mechanism via a nonequilibrium state}

Although the experiments in Sec. VI.C.4 showed the intriguing possibility of triggering magnetization reversal with a femtosecond stimulus, the relevant time scales and mechanism of such an optically induced magnetization reversal are unclear, since a precessional switching within 40 fs would require enormous effective magnetic fields above $10^{2} \mathrm{~T}$ and unrealistically strong damping. To clarify the dynamics of the switching process, femtosecond single-shot time-resolved optical imaging of magnetic domains was used together with multiscale modeling beyond the macrospin approximation (Vahaplar et al., 2009).

GdFeCo samples were excited by a single circularly polarized laser pulse. A single linearly polarized probe pulse delayed with respect to the pump was used for ultrafast imaging of the magnetic domain structure via the magneto-optical Faraday effect. Magnetic domains with magnetization parallel (up) or antiparallel (down) to the sample normal are seen as white or black regions, respectively, in an image on a charge-coupled device camera. After each write-read event, the initial magnetic state was restored by applying a magnetic field pulse. Taking images of the magnetic structure for different delays between the pump and probe pulses, we were able to visualize the ultrafast dynamics of the laserinduced magnetic changes in the material.

Figure 46 shows images of magnetic domains at different delays after excitation by right-handed $\left(\sigma^{+}\right)$or lefthanded $\left(\sigma^{-}\right)$circularly polarized pulses. In the first few hundreds of femtoseconds, pump pulses of both helicities bring the originally magnetized medium into a strongly nonequilibrium state with no measurable net magnetization, seen as a gray area in the second column of Fig. 46, the size of which is given by the laser beam diameter and the intensity profile. In the following few tens of picoseconds either the medium relaxes back to the initial state or a small $(\sim 5 \mu \mathrm{m})$ domain with a reversed magnetization is formed. It is thus obvious that (i) switching proceeds via a strongly nonequilibrium demagnetized state, clearly not following the conventional route of precessional motion and (ii) the final state is defined by the helicity of the $100 \mathrm{fs}$ pump pulse (last column of Fig. 46).

The switching time is in fact surprising because in contrast to heat-assisted magnetic recording (Hohlfeld et al., 2001), the reversal time is much longer than the effective light-induced magnetic field pulse $H_{\text {eff }}$. The duration of the latter $\Delta t_{\text {eff }}$ is still an open question but can be different from the full width at half maximum (FWHM) of the optical pulse. However, $\Delta t_{\text {eff }}$ can be estimated from the spectrum of terahertz radiation generated by an Fe film excited by a subpicosecond visible laser pulse. Based on a half-period oscillation with the lowest frequency in the spectrum reported by Bartelt et al. (2007), the maximum $\Delta t_{\text {eff }}$ is about 3 ps. The pulse amplitude $H_{\text {eff }}$, for a typical pump fluence of $2.5 \mathrm{~J} / \mathrm{m}^{2}$ and the magneto-optical constant of $\mathrm{GdFeCo}\left(\sim 3 \times 10^{5} \mathrm{deg} / \mathrm{cm}\right)$, reaches $20 \mathrm{~T}$.

To understand this reversal process the LandauLifshitz-Bloch equation was solved. This macrospin approach encapsulates well the response of a set of coupled atomic spins subjected to rapidly varying temperature changes, including the reduction in the magnitude of $\mathbf{M}$; see Sec. II.B. The temperature-dependent parameters for the LLB equation, i.e., the longitudinal and transverse susceptibilities and the temperature variation in the magnetization, were calculated atomistically using Langevin dynamics combined with a LandauLifshitz-Gilbert equation for each spin (Kazantseva, Hinzke, et al., 2008). The laser-induced increase in the kinetic energy (temperature) of the electrons was simulated using a two-temperature model (Kazantseva, Nowak, et al., 2008), the parameters for which were taken to be typical for a metal (Zhang, Hübner, et al., 2002) (electron heat capacity $C_{e}=1.8 \times 10^{6} \mathrm{~J} / \mathrm{m}^{3} \mathrm{~K}$ at room temperature and electron-phonon coupling $G_{\text {el-ph }}$ $=1.7 \times 10^{18} \mathrm{~J} / \mathrm{K} \mathrm{s}$ ). The simulations showed that in the first $100 \mathrm{fs}$ the electron temperature $T_{\mathrm{el}}$ increases from $300 \mathrm{~K}$ up to $T_{\mathrm{el}}^{*}$ and relaxes with a time constant of $0.5 \mathrm{ps}$ down to the vicinity of $T_{C}$. Simultaneously the spins experience a short pulse of effective magnetic field with amplitude $H_{\text {eff }}=20 \mathrm{~T}$ and duration $\Delta t_{\text {eff. }}$ The possibility of magnetization reversal under these circumstances has been analyzed numerically for a volume of $(30 \mathrm{~nm})^{3}$. The results of the simulations are plotted in Fig. 47(a) as a phase diagram, defining the combinations of $T_{\mathrm{el}}^{*}$ and $\Delta t_{\mathrm{eff}}$ for which switching occurs for the given $H_{\text {eff. }}$ As can be seen from the diagram, a field duration as short as $\Delta t_{\text {eff }}=250 \mathrm{fs}$ can reverse the magnetization. For better insight into the reversal process, we simulated the latter for $\Delta t_{\mathrm{eff}}=250 \mathrm{fs}$ and $T_{\mathrm{el}}^{*}=1130 \mathrm{~K}$. The result is 

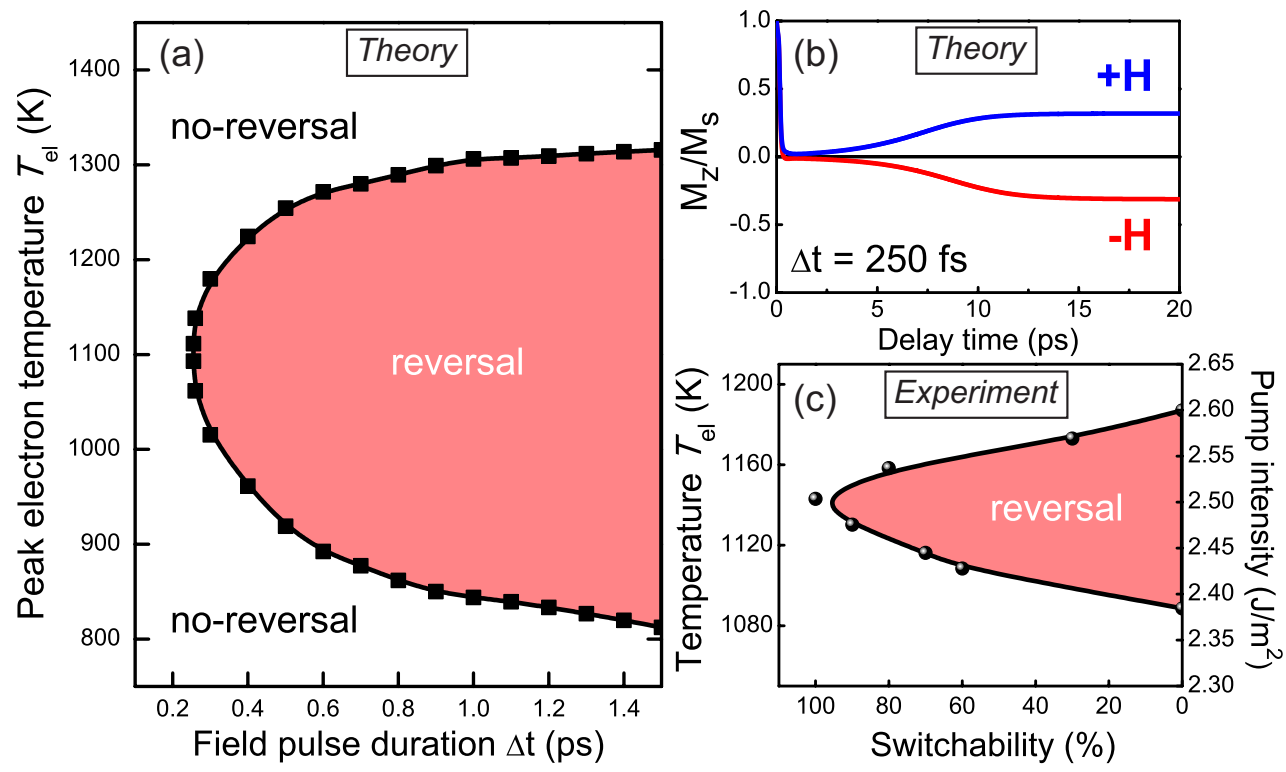

FIG. 47. (Color online) Magnetization reversal triggered by a combined action of a short magnetic field pulse $H_{\text {eff }}$ and ultrafast heating of electrons to a temperature of $T_{\mathrm{el}}^{*}$. (a) Phase diagram showing the magnetic state of the $(30 \mathrm{~nm})^{3}$ volume achieved within $10 \mathrm{ps}$ after the action of the optomagnetic pulse with parameters $H_{\mathrm{eff}}=20 \mathrm{~T}, \Delta t_{\mathrm{eff}}$, and $T_{\mathrm{el}}^{*}$. (b) The averaged $z$ component of the magnetization vs delay time as calculated for $250 \mathrm{fs}$ magnetic field pulses $H_{\mathrm{eff}}=20 \mathrm{~T}$ and $T_{\mathrm{el}}^{*}=1130 \mathrm{~K}$. (c) Switchability vs the pump intensity for $\mathrm{Gd}_{22} \mathrm{Fe}_{68.2} \mathrm{Co}_{9.8}$ at room temperature. Peak electron temperature $T_{\mathrm{el}}^{*}$ is calculated using $C_{e}$. Note that in this range of intensities the amplitude of the effective light-induced magnetic field varies within 19.2-20.8 T. From Vahaplar et al., 2009.

plotted in Fig. 47(b), showing that already after $250 \mathrm{fs}$ the effective fields of two different polarities bring the medium into two different states, while the magnetization is nearly quenched within less than 0.5 ps. This is followed by relaxation either to the initial state or to the state with reversed magnetization, achieved already within $10 \mathrm{ps}$. The considered pulse duration $\Delta t_{\mathrm{eff}}$ of $250 \mathrm{fs}$ is only 2.5 times larger than the FWHM of the optical pulse in the experiments and well within the estimated lifetime of a medium excitation responsible for $\mathrm{H}_{\text {eff. }}$ An important result is that in simulations $\Delta t_{\text {eff }}$ was found to be sensitive to the parameters of the twotemperature model. In particular, an increase in $G_{\text {el-ph }}$ leads to a reduction in the minimum field pulse duration. This shows that the suggested mechanism may, in principle, explain the experimentally observed laser-induced magnetization reversal. This magnetization reversal does not involve precession; instead, it occurs via a linear reversal mechanism, where the magnetization first vanishes and then reappears in the direction of $H_{\text {eff }}$, avoiding any transverse magnetization components, as shown in Fig. 46. Exactly as in the experiments, the initial 250 fs effective magnetic field pulse drives the reversal process, which takes one to two orders of magnitude longer.

The state of magnetization after the pulse is critically dependent on the peak temperature $T_{\mathrm{el}}^{*}$ and the pulse duration. For ultrafast (linear) reversal by a 250 fs field pulse it is necessary that, within this time, $T_{\mathrm{el}}^{*}$ reaches the vicinity of $T_{C}$. If, however, this temperature is too high and persists above $T_{C}$ for too long, the reversed magnetization is destroyed and the effect of the helicity is lost. This leads to a phase diagram [Fig. 47(a)], showing that the magnetization reversal may occur in a certain range of $T_{\mathrm{el}}^{*}$. Such a theoretically predicted reversal window of electron temperature can be easily verified in the experiment when one changes the intensity of the laser pulse. Figure $47(\mathrm{c})$ shows the switchability, i.e., the difference between the final states of magnetization achieved in the experiment with $\sigma^{+}$- and $\sigma^{-}$-polarized pulses, as a function of $T_{\mathrm{el}}^{*}$, calculated from the laser pulse intensity. It is seen that, indeed, switching occurs within a fairly narrow laser intensity range. For intensities below this window no laser-induced magnetization reversal occurs, while if the intensity exceeds a certain level both helicities result in magnetization reversal since the laser pulse destroys the magnetic order completely, which is then reconstructed by stray fields (Ogasawara et al., 2009). Despite this qualitative agreement between simulations and experiments, the experimentally observed reversal time is several times larger than the calculated 10 ps. The latter, however, is calculated for a $30 \mathrm{~nm}$ domain whereas in our experiments the magnetization in a $5 \mu \mathrm{m}$ spot is manipulated.

\section{Excitation of the magnetization dynamics with linearly polarized light}

As shown above in many examples, the phase of the optically excited spin precession is unambiguously defined by the helicity of the laser pulses. This fact actually created certain controversies in the description of the phenomena, namely, about the role of the angular momentum of the photons in the process. Thus, a complete understanding of the processes taking place during and after the optical excitation by a subpicosecond laser 
pulse is of particular importance. Here we expand the discussion and describe the nonthermal optomagnetic effects with linearly polarized pump pulses (Kalashnikova et al., 2007, 2008), measured in iron borate $\left(\mathrm{FeBO}_{3}\right)$ that possesses a strong magneto-optical response, both linear (Faraday effect) and quadratic (MLD) in the magnetization.

\section{Detection of the FMR mode via magnetic linear birefringence in $\mathrm{FeBO}_{3}$}

Iron borate $\left(\mathrm{FeBO}_{3}\right)$ crystallizes in the rhombohedral calcite-type crystallographic structure (space group $R \overline{3} c$ ) with two formula units per unit cell (Bernal et al., 1963; Diehl, 1975; Wijn, 1994). Below the Néel temperature $T_{N}=348 \mathrm{~K}$, the magnetic moments $\mathbf{M}_{1}$ and $\mathbf{M}_{2}$ of the two $\mathrm{Fe}^{3+}$ sublattices are coupled antiferromagnetically. It is common to describe such magnetic structure in terms of the ferromagnetic vector $\mathbf{M}=\mathbf{M}_{1}+\mathbf{M}_{2}$ and the antiferromagnetic vector $\mathbf{L}=\mathbf{M}_{1}-\mathbf{M}_{2}$. The magnetocrystalline anisotropy of $\mathrm{FeBO}_{3}$ corresponds to an "easyplane" type of spin alignment, where spins of both sublattices are perpendicular to the crystallographic $z$ axis. The effective magnetocrystalline anisotropy field is $H_{A}$ $\simeq 1.7 \mathrm{kOe}$ (Schober, 1976). The presence of the antisymmetric exchange interactions (Dzyaloshinskii, 1957; Moriya, 1960) leads to a canting of the spins in the $x-y$ plane. The effective Dzyaloshinskii field is $H_{D}$ $\simeq 61.9 \mathrm{kOe}$ (Schober, 1976), corresponding to a canting angle of $\sim H_{D} / H_{E} \simeq 1^{\circ}$ and a net magnetic moment of $4 \pi M_{s}=115 \mathrm{G}$ at $T=300 \mathrm{~K}$ (Kurtzig et al., 1969) (238 G at $T \rightarrow 0 \mathrm{~K})$. The magnetocrystalline anisotropy in the $x$ - $y$ plane is as weak as $H_{A^{\prime}} \simeq 0.26$ Oe (Schober, 1976).

The optically excited spin precession in $\mathrm{FeBO}_{3}$ was studied by means of a magneto-optical pump-probe technique (see Sec. III.A) at photon energy of $E_{0}$ $=1.54 \mathrm{eV}$. Two experimental geometries were used: the magnetic field was always applied along the $x$ axis, while the pump pulses were propagating along either the $z$ or the $y$ axis.

The spin precession induced by the pump pulses leads to a perturbation of the dielectric permittivity tensor (see Sec. III.B) which, in turn, leads to a change in the polarization of the probe beam via either the Faraday effect or magnetic linear birefringence.

The rotation of the probe polarization as a function of time delay between pump (propagating along the $z$ axis) and probe pulses is shown in Fig. 48(a) for different values of the applied magnetic field. Clear field-dependent oscillations are observed in these data. In Fig. 48(b) the dependence of the oscillation frequency on the magnetic field is plotted. This dependence is in good agreement with the behavior of the FMR mode in $\mathrm{FeBO}_{3}$ (Jantz et al., 1976). Also the dependence of the frequency of the oscillations on temperature [Fig. 48(d)] is consistent with the temperature behavior that one would expect for the FMR mode. Thus these data show that the laser pulses propagating along the $z$ axis excite the FMR mode of coherent spin precession in $\mathrm{FeBO}_{3}$.

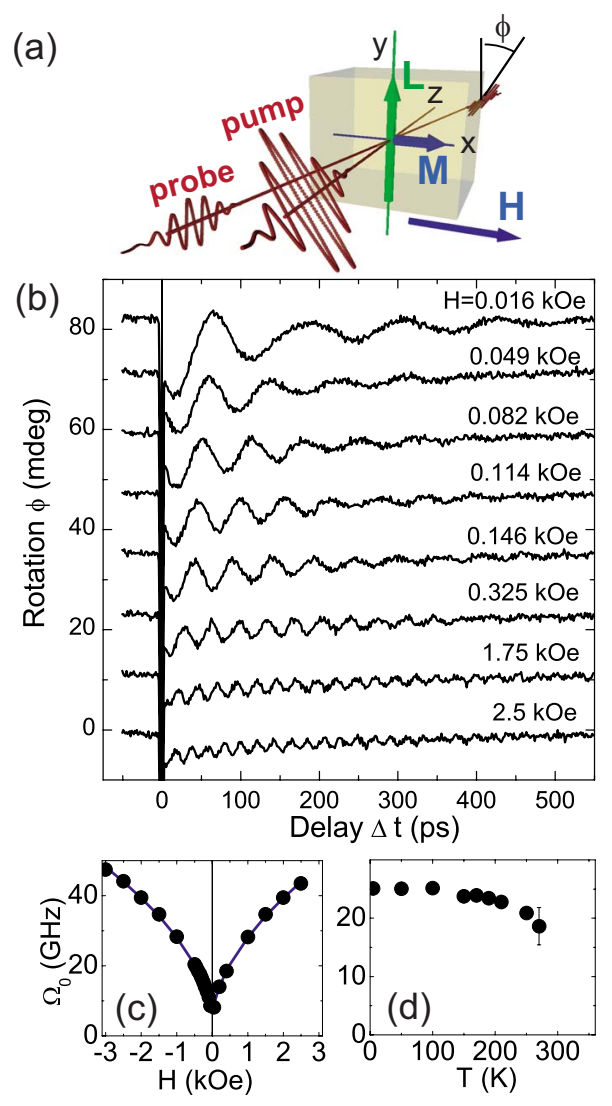

FIG. 48. (Color online) Excitation and detection of the ferromagnetic mode of spin precession by linearly polarized pump pulses. (a) Experimental geometry. (b) Pump-induced rotation of the probe polarization $\phi$ as a function of time delay between linearly polarized pump and probe pulses for different values of the applied magnetic field. (c) The frequency $\Omega_{0}$ of the observed oscillations as a function of the applied field strength $H$. (d) The frequency $\Omega_{0}$ of the oscillations as a function of temperature $T$. The results were obtained for a pump intensity of $10 \mathrm{~mJ} / \mathrm{cm}^{2}$. Pump pulses were linearly polarized with azimuthal angle $\theta=45^{\circ}$. Results in (b) and (c) are obtained at a temperature $T=10 \mathrm{~K}$. From Kalashnikova et al., 2008.

The FMR mode supposes oscillations of the $l_{x}, m_{y}$, and $m_{z}$ components of $\mathbf{I}(\mathrm{t})$ and $\mathbf{m}(\mathrm{t})$. There are various magneto-optical effects that can serve as a probe of such a precession. That is, in the experimental geometry shown in Fig. 48(a), the spin precession may lead to a transient rotation of the probe polarization via both the Faraday effect and the magnetic linear birefringence (MLB). For instance, $m_{z}(t)$ can be detected using the Faraday effect with the probe polarization rotation $\phi(t)$ given by

$$
\phi_{F}(t)=\omega_{0} d \frac{\delta \varepsilon_{x y}^{a}(t)}{n}=\omega_{0} d \frac{K m_{z}(t)}{n}
$$

where $\omega_{0}$ is the pulse central frequency, $d$ is the sample thickness, and $n$ is the refractive index. In turn, $l_{x}(t)$ oscillations cause MLB, which also leads to a rotation of the probe polarization, 

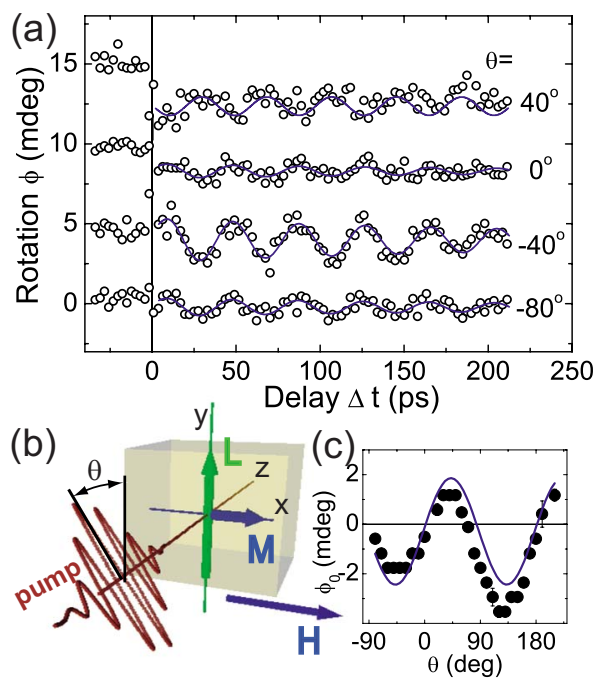

FIG. 49. (Color online) Laser-induced magneto-optical response from $\mathrm{FeBO}_{3}$ : (a) Oscillations of the probe polarization as a function of the time delay between linearly polarized pump and probe pulses for different orientations of the pump polarization. The pump pulses propagate along the $z$ axis shown in (b). The probe pulse is not shown in (b) for simplicity. (c) The amplitude of oscillations as a function of the pump polarization azimuthal angle (symbols) and its fit using Eq. (71) (line). The results are obtained at $T=10 \mathrm{~K}, H=1.75 \mathrm{kOe}$, and $I=10 \mathrm{~mJ} / \mathrm{cm}^{2}$. From Kalashnikova et al., 2008.

$$
\phi_{\mathrm{MLB}}(t)=\omega_{0} d \frac{\delta \varepsilon_{x y}^{s}(t)}{n} \approx \omega_{0} d \frac{G L_{y}^{(0)} l_{x}(t) \cos (2 \xi)}{n},
$$

where $G=b_{1}-b_{2}$ is the magneto-optical coefficient and $\xi$ is the incoming polarization of the probe pulse. In the case of the Faraday effect, the measured signal $\phi(t)$ would not depend on the incoming polarization. Note that, in the given geometry, it is the symmetric part of the off-diagonal tensor components which is most affected by the FMR mode of precession [linear with respect to $\left.l_{x}(t)\right]$. The change in the diagonal components is quadratic with respect to $l_{x}(t)$ and can be neglected. Experimentally, a clear $180^{\circ}$ dependence of the signal on $\xi$ was observed. Therefore the measured signal originates from the transient MLB [Eq. (70)] and reveals an inplane motion of the antiferromagnetic vector $\mathbf{L}$. The fact that MLB dominates over the Faraday effect is caused by the strong ellipticity of the FMR mode of spin precession: since the magneto-optical constants $K$ and $G$ are comparable for the photon energy $E=1.54 \mathrm{eV}$, the ratio between the transient Faraday effect and MLB is mainly defined by the ratio of the dynamic components of magnetic vectors $m_{z} / l_{x}$ that is as small as 0.01 because of strong easy-plane-type anisotropy.

\section{Excitation of coherent magnons by linearly and circularly polarized pump pulses}

Figure 49(a) shows the spin precession excited by linearly polarized pump pulses for various azimuthal orientations of the pump polarization $\theta$, as shown in Fig. (a)

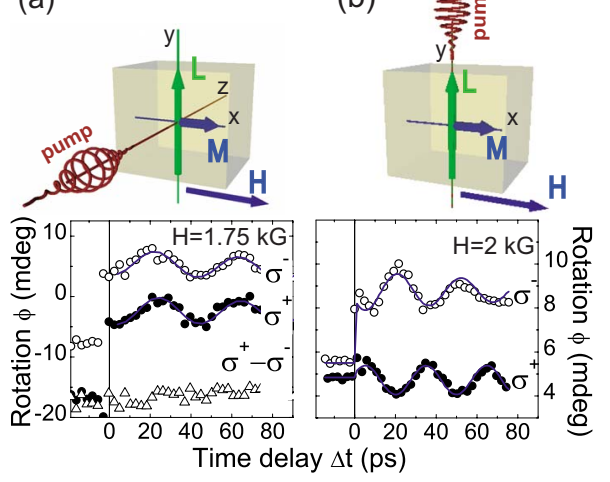

FIG. 50. (Color online) Spin precession excited by circularly polarized pump pulses propagating along (a) the $z$ axis and along (b) the $y$ axis. $\sigma^{+}-\sigma^{-}$is the difference between the spin precession amplitude excited by right- and left-handed circularly polarized pump pulses. In both cases the magnetic field is applied along the $x$ axis and the probe is at $10^{\circ}$ from the pump propagation direction. The results are obtained at $T=10 \mathrm{~K}$ and $I=10 \mathrm{~mJ} / \mathrm{cm}^{2}$. From Kalashnikova et al., 2008.

49(b). The spin precession amplitude depends on $\theta$ [Fig. 49(c)].

The effect of circularly polarized pulses appeared to depend on the mutual orientation of the pump propagation direction and antiferromagnetic vector $L_{y}^{(0)}$. As can be seen from Fig. 50(a), the circularly polarized pump pulses propagating along the $z$ axis do excite spin precession but a change in their helicity affects neither the amplitude nor the phase of the oscillations $\left(\phi^{\sigma^{+}}-\phi^{\sigma^{-}}\right.$ $=0$ ). In contrast, the spin precession excited by circularly polarized pump pulses propagating along the $y$ axis changes phase by $180^{\circ}$ when the pump helicity of the light is reversed [Fig. 50(b)]. We previously explained how circularly polarized pulses act on the spins as effective magnetic field pulses with a direction depending on the helicity. The phase of the excited precession, therefore, should be defined by the helicity of the pump pulses. This inverse Faraday effect is determined by the same magneto-optical susceptibility that also accounts for the Faraday effect and is expected to be allowed in media of any symmetry. Therefore, the absence of this effect in the results shown in Fig. 50(a) is, at first glance, puzzling considering the fact that the Faraday effect in $\mathrm{FeBO}_{3}$ is one of the strongest among the iron oxides (Kurtzig et al., 1969). Note that the incompleteness of such an interpretation was pointed out by (Woodford, 2007).

Below we show that this together with the observed polarization dependence of the excitation can be explained by taking into account the strongly elliptical character of the spin precession modes in $\mathrm{FeBO}_{3}$. That is, it is not only the strength of the generated effective field that plays a role but also susceptibility of the system to that field.

\section{ISRS as the mechanism of coherent magnon excitation}

We first consider the excitation of spin precession by the linearly polarized pump pulses propagating along 

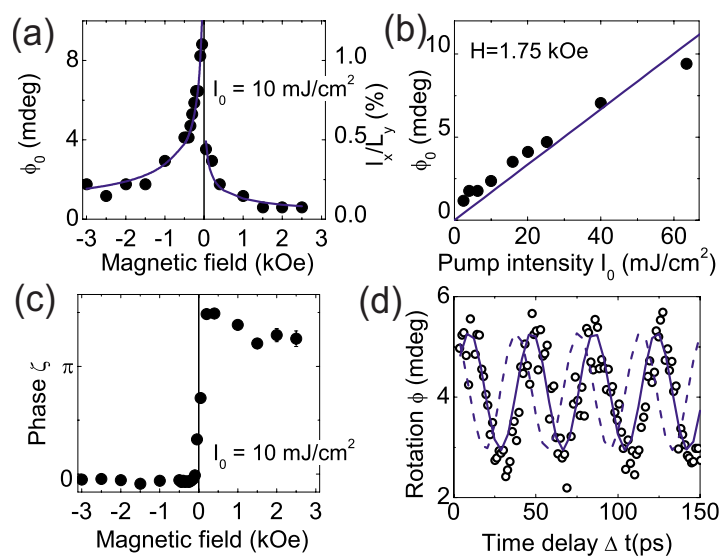

FIG. 51. (Color online) Experimental dependencies of the oscillations amplitude $\phi_{0}$ (dots) on (a) the magnetic field $H$ and (b) pump intensity $I_{0}$. Solid lines in (a) and (b) represent the dependencies described by Eq. (71). (c) Initial phase $\zeta$ of the pump-induced oscillations as a function of the magnetic field $H$ when described by $\phi(\Delta t)=\phi_{0} \sin \left(\Omega_{0} \Delta t+\zeta\right)$. A typical experimental curve $\phi(\Delta t)$ is shown (circles) in (d). For reference, the curves $\phi(\Delta t)=\phi_{0} \sin \left(\Omega_{0} \Delta t+0\right)$ (solid line) and $\phi(\Delta t)$ $=\phi_{0} \sin \left(\Omega_{0} \Delta t+\pi / 2\right)$ (dashed line) are shown. The experimental results shown in (a)-(d) are obtained for the pump polarization $\theta=45^{\circ}$ and temperature $T=10 \mathrm{~K}$. From Kalashnikova et al., 2008 .

the $z$ axis [Fig. 48(a)]. Combining Eqs. (65), (66), and (70) (see Sec. VI.A.3) for the transient rotation of the probe polarization caused by magnons excited via ISRS, we obtain

$$
\phi^{\operatorname{lin}}(t)=A \frac{I_{0}}{4 n c} G L_{y}^{(0) 2} a_{x}^{2} \sin 2 \theta \sin \Omega_{0} t
$$

where $A=\omega_{0} d / n, \theta$ is the azimuthal angle of the pump polarization, $\Omega_{0}$ is the FMR frequency, $G=b_{1}-b_{2}$ is the magneto-optical coefficient, $I_{0}$ is the integrated pump pulse intensity, $\omega_{0}$ is the pump pulse central frequency, and $n$ is the refraction index at $\omega_{0}$.

Comparison of Eq. (71) with the experimental results shows good agreement. In particular, the experimentally obtained dependence of the oscillation amplitude on the applied magnetic field $H$ [Fig. 51(a)] is described by $\phi^{\operatorname{lin}}(H) \sim a_{x}^{2} \sim 1 / \Omega_{0} \sim 1 / \sqrt{H}$, following Eq. (71). The pump-induced oscillations of the probe polarization should, according to Eq. (71), possess a sinelike behavior in the time domain, which is, indeed, observed in the experiment [Figs. 51(b) and 51(d)] for the magnetically saturated sample. The theoretically predicted dependence of the oscillation amplitude on the polarization of the pump pulses $\phi^{\operatorname{lin}}(\theta) \sim \sin 2 \theta$ shows good agreement with our experimental data [Fig. 49(c)].

In the case of circularly polarized pump pulses, the transient rotation of the probe polarization excited by pulses propagating along the $z$ and $y$ axes, respectively, can be expressed as

$$
\begin{aligned}
& \phi^{\sigma^{ \pm} \| z}(t)= \pm A G L_{y}^{(0)} a_{x}\left(\frac{I_{0}}{4 n c} K b_{z} \cos \Omega_{0} t\right) \sim b_{z} a_{x}, \\
& \phi^{\sigma^{ \pm} \| y}(t)= \pm A K_{2}\left(\frac{I_{0}}{4 n c}\left(K_{1} b_{y}+K_{2} a_{x}\right) a_{x} \sin \Omega_{0} t\right) \sim a_{x}^{2} .
\end{aligned}
$$

Now, keeping in mind that the FMR precession possesses strong ellipticity, i.e., $b_{z} \ll a_{x}$, we obtain that $\phi^{\sigma^{ \pm} \| z} \ll \phi^{\sigma^{ \pm} \| y} \simeq \phi^{\operatorname{lin} \| z}$. Our experimental data (Fig. 50) show that, indeed, circularly polarized laser pulses effectively excite a helicity-dependent spin precession only when propagating along the $y$ axis. It is worth noting that these results are in perfect agreement with the observation of spontaneous Raman scattering in $\mathrm{FeBO}_{3}$ reported by Jantz et al. (1976). It was shown there that, because of spin precession ellipticity, the scattering of light propagating along the $z$ axis is defined mainly by the second-order magneto-optical constant [i.e., by $G$ in Eq. (71)], while the scattering of light propagating along the $y$ axis is defined by a first-order magneto-optical constant [i.e., by $K_{1}$ and $K_{2}$ in Eq. (72b)]. A similar effect of spin precession ellipticity on the Raman scattering intensity was reported by White et al. (1982) for orthoferrites.

Here we comment on the distinction between these results and those discussed in Sec. V.A.1. There the excitation of coherent spin precession by linearly polarized laser pulses in ferrimagnetic garnet films was due to a quasistationary photoinduced change in magnetic anisotropy. The oscillations of $l_{x}(t)$ and, consequently, $\phi(t)$ excited via such a process should obey the $\left(1-\cos \Omega_{0} t\right)$-like dependence on the time delay between pump and probe pulses. Here $\phi(t) \sim l_{x}(t) \sim \sin \Omega_{0} t$ [see Figs. 48(a), 49(a), and 51(b)]. In addition, the dependence of the amplitude of the excited precession on the pump pulse intensity [Fig. 51(d)] is also different from the one due to the photomagnetic effects (Hansteen et $a l ., 2006)$, where the photoinduced change is related to the absorption by impurity centers. As their concentration is limited, the dependence of the excited spin precession amplitude on the pump intensity indeed showed saturation. However, no saturation of the spin precession amplitude with pump intensity was observed in the present case [Fig. 51(b)].

Thus, the experimentally observed excitation of coherent magnons in $\mathrm{FeBO}_{3}$ can be unambiguously described in terms of impulsive stimulated Raman scattering. The efficiency of the excitation by the pump pulses with certain polarization is defined by the ellipticity of the magnon mode.

It is therefore interesting and important to clarify the approach of the ultrafast inverse Faraday effect used previously to describe the light-induced spin precession in orthoferrites, garnets, and metallic alloys (Kimel et al., 2007) and to compare it with this ISRS-based interpretation. 


\section{Effective light-induced field approach}

The excitation of coherent spin precession by short laser pulses has been described using the approach in which light acts on spins as an effective light-induced magnetic field $\mathbf{H}^{\mathrm{eff}}=K\left[\mathbf{E} \times \mathbf{E}^{*}\right]$. Strictly speaking, this was originally defined for a cubic paramagnetic medium (Pershan et al., 1966). To describe the impulsive action of light on the spins of a multisublattice medium, we introduce the light-induced effective fields

$$
\mathbf{H}_{i}^{\mathrm{eff}}=-\partial \mathcal{H}_{i, \text { int }} / \partial \mathbf{M}_{i},
$$

where $\mathbf{M}_{i}$ is the sublattice magnetization and $\mathcal{H}_{i \text {,int }}$ is the Hamiltonian describing the interaction of light with the $i$ th sublattice. For the case of a one-sublattice ferromagnetic medium, the further description of the light-matter interaction is trivial because one ferromagnetic vector $\mathbf{M}$ is sufficient to describe the collective response of the magnetic system to the light action (see Sec. VI.A.2). A multisublattice magnetic medium, however, is described by several magnetic vectors $\mathbf{M}$ and $\mathbf{L}_{j}$, where $j=1, \ldots, n$ -1 and $n$ is the number of magnetic sublattices. In particular, $\mathrm{FeBO}_{3}$ has two magnetic sublattices with magnetizations $\mathbf{M}_{1}$ and $\mathbf{M}_{2}$. The interaction of light with each sublattice can be described by the effective field $\mathbf{H}_{1}^{\text {eff }}$ $=-\partial \mathcal{H}_{1, \text { int }} / \partial \mathbf{M}_{1}$ and $\mathbf{H}_{2}^{\text {eff }}=-\partial \mathcal{H}_{2 \text {,int }} / \partial \mathbf{M}_{2}$. Making a transition from the sublattice magnetizations to the ferromagnetic $\mathbf{M}=\mathbf{M}_{1}+\mathbf{M}_{2}$ and antiferromagnetic $\mathbf{L}=\mathbf{M}_{1}-\mathbf{M}_{2}$ vectors, one obtains two effective fields

$$
\begin{aligned}
& \mathbf{H}^{\text {eff }}=-\partial \mathcal{H}_{\text {int }} / \partial \mathbf{M}, \\
& \mathbf{h}^{\text {eff }}=-\partial \mathcal{H}_{\text {int }} / \partial \mathbf{L},
\end{aligned}
$$

which can also be understood as $\mathbf{H}^{\text {eff }}=\mathbf{H}_{1}^{\text {eff }}+\mathbf{H}_{2}^{\text {eff }}$ and $\mathbf{h}^{\text {eff }}=\mathbf{H}_{1}^{\text {eff }}-\mathbf{H}_{2}^{\text {eff }}$. The latter field accounts for the nonequivalent responses of the $\mathrm{Fe}^{3+}$ ions at different crystallographic positions to the action of light. It can be shown that it is this field $\mathbf{h}^{\text {eff }}$ that induces the spin precession in a weak ferromagnet, such as $\mathrm{FeBO}_{3}$. Note that, in general, for a medium with $n$ magnetic sublattices there are $n-1$ fields $\mathbf{h}_{j}^{\text {eff }}=-\partial \mathcal{H}_{\text {int }} / \partial \mathbf{L}_{j}$.

A detailed analysis of the response of the $\mathrm{FeBO}_{3}$ spin system reveals that the efficiency of the excitation by either linearly or circularly polarized optical pulses is determined by the details of the magnetic structures. In particular, the effect of a circularly polarized pulse propagating along the $y$ axis resembles that of a linearly polarized one propagating along the $z$ axis. Namely, the effective field induced by the former and latter pulses is $h_{x}^{\text {eff }}$ and the spins move out of the $x-y$ plane during the pulse. In analogy to the inverse Faraday effect, the appearance of an effective fields $\mathbf{H}^{\mathrm{eff}}$ and $\mathbf{h}^{\mathrm{eff}}$ generated by linearly polarized laser pulses can be seen as a manifestation of the inverse Cotton-Mouton effect (Zon and Kupershmidt, 1983).

Finally, we note that the approaches based on the equation of motion for magnon normal coordinates (Secs. VI.A and VI.D.3) and on the LL equations are equivalent for the treatment of the experimental results presented in this section. However, the latter approach will be more convenient for the description of large deviations or even switching (Stanciu, Hansteen, et al., 2007) of spins caused by strong laser pulses, when the equation of motion [Eq. (49)] becomes highly nonlinear.

\section{CONCLUSIONS AND OUTLOOK}

Optical manipulation of magnetic order by femtosecond laser pulses has developed into an exciting and still expanding research field that keeps being fueled by a continuous stream of new and sometimes counterintuitive results. The discovery of ultrafast demagnetization by a $60 \mathrm{fs}$ laser pulse, that is, demagnetization on a time scale much faster than the spin-lattice relaxation time, has triggered a wealth of experimental and theoretical research focused on the questions: How fast and by what means can magnetic order be changed and manipulated? What are the channels of energy and angular momentum transfer between the photons and the spins and the various other degrees of freedom?

The goal of this review was to describe the progress in our understanding of femtosecond laser manipulation of magnetic order in the past 13 years after the original discovery with a systematic review of the results obtained in a broad class of materials that includes both ferromagnetically and antiferromagnetically ordered metallic, semiconducting, and dielectric materials. As a magnetic medium is characterized by a specific magnetic ordering, resulting from the interactions among electrons, spins, and the lattice, such a systematic approach may help to reveal the roles of the electronic band structure, the spin-orbit coupling, and the magnetic structure in this process. An excitation by a femtosecond laser pulse brings a medium into a strongly nonequilibrium state, where a conventional description of magnetization in terms of thermodynamics is no longer valid. The interactions between the relevant reservoirs are described by the spin-orbit, spin-lattice, and electron-phonon interactions, which span a time scale from picoseconds to nanoseconds within these various materials, while electron-electron and exchange interactions set the lower boundary of the possible time scales.

The role of the electronic structure is demonstrated by a comparison of the demagnetization rates between metals and dielectrics: the observed ultrafast (subpicosecond) demagnetization in metals can be understood by the heating of the itinerant electrons by the optical (1-2 eV) laser pulse. The hot electron gas plays the role of a thermal bath for spins and thus facilitates both an intensification of spin-flip processes and the demagnetization. In contrast, in dielectrics, even the electrons that are excited across the band gap cannot gain a lot of kinetic energy and will thus not contribute in the process of demagnetization. There the latter occurs via laserinduced heating of the lattice, after the spins are heated via the spin-lattice interaction on a time scale of a 100 ps.

Even without itinerant electrons, however, magnetization can be manipulated on a time scale faster than the 
spin-lattice relaxation by optical manipulation of the magnetic anisotropy. It has been shown that this can be done either via laser-induced heating, using the mostly more (i.e., than spin-lattice) effective electron-phonon interaction $(\sim 1 \mathrm{ps})$, or via direct (photomagnetic) optical pumping of specific electronic transitions. The latter effects have been mainly observed in materials with low conductivity and long excited-state lifetimes, i.e., semiconductors and dielectrics; the lifetime of this effect is expected to be very short in metals (Gamble et al., 2009).

In the past five years a totally new, so-called optomagnetic approach was introduced that depended on the presence of strong spin-orbit interaction in the ground or excited electronic states. The corresponding magnetooptical susceptibility that is responsible for the magnetooptical Faraday effect, i.e., the effect of the magnetization on the polarization of light, was demonstrated to be also responsible for the inverse Faraday effect: the manipulation of the magnetization by circularly polarized light. Because of this optomagnetic effect, an intense femtosecond circularly polarized optical laser pulse was demonstrated to act on spins similarly to an equivalently short effective magnetic field pulse with a strength up to $20 \mathrm{~T}$. With such an optically induced magnetic field, one may selectively excite different modes of magnetic resonance, realize quantum control of spin oscillations, and trigger phase transitions nonthermally on a subpicosecond time scale. It was shown that a single 40 fs circularly polarized laser pulse can controllably reverse magnetization, where the direction of this switching was solely determined by the helicity of the light pulse.

These results demonstrated that femtosecond laser pulses are indeed novel and effective stimuli for the manipulation of magnetic order. Recent results that show that even linearly polarized laser pulses can lead to similar effects have indicated the importance of the details of the magnetic structure for the understanding of these optomagnetic results.

An additional comment should be made about the interpretation of the experimental results. Soon after the original discovery of ultrafast demagnetization via the observation of an ultrafast quenching of the magnetooptical Kerr signal, questions arose about the validity of the use of magneto-optical probes to follow changes in magnetic order at such short time scales. Recent theoretical work shows, however, that the correspondence between optical and magneto-optical response sensitively depends on wavelength and pulse length, with a maximum delay between the two on the order of tens of femtoseconds (Zhang et al., 2009). There have been several attempts to describe ultrafast laser-induced spin dynamics using $a b$ initio theory and atomistic and micromagnetic simulations. None of these approaches has so far been successful in providing a complete theoretical framework, suggesting that a solution might be found in an interdisciplinary multiscale approach.

The same can be said for the experimental approaches: for a further and detailed understanding of "femtomagnetism," a combination of experimental approaches such as optical excitation and x-ray probing will allow a nanometer-scale picture to be obtained of what actually happens during and after the excitation of a magnetic medium by a femtosecond laser pulse (Stamm et al., 2007; Lorenc et al., 2009). The development of novel femtosecond slicing approaches at synchrotron sources is therefore very promising. Moreover, this challenge is one of the motivations for the development of the next generation of $\mathrm{x}$-ray sources-x-ray free electron lasers able to generate intense subpicosecond pulses of tunable x-ray radiation.

It may also be expected that the further progress in the laser control of magnetism will be associated with pushing the duration of the optical stimulus down to the attosecond time scale. In this time domain, owing to the coherence of optical excitations, the interaction of light with solids will drastically depend on the shape and duration of the laser pulse creating thus the new degrees of freedom.

Moreover, considering the progress in the development of compact ultrafast lasers (Keller, 2003), optical control of magnetic order may potentially revolutionize data storage and information processing technologies. Regarding these potential applications, for that it will be essential to extend the present state of optical manipulation and control of magnetic order toward smaller nanoscale dimensions. Given the rapid developments in nano-optics and plasmonics, such possibilities do not seem to be too far fetched. Either way, the present review shows that "femtomagnetism" is a vibrant new field with a wealth of new phenomena waiting to be discovered and explored.

\section{ACKNOWLEDGMENTS}

This review has been possible only through the dedicated work of our co-workers A. M. Balbashov, F. Hansteen, A. Itoh, A. M. Kalashnikova, C. D. Stanciu, A. Tsukamoto, P. A. Usachev, and K. Vahaplar, supplemented by daily technical support of A. J. Toonen and A. F. van Etteger. We are grateful to M. Aeschlimann, C. H. Back, J.-Y. Bigot, U. Bovensiepen, R. Chantrell, H. Dürr, V. N. Gridnev, B. A. Ivanov, M. I. Katsnelson, B. Koopmans, D. Mazurenko, A. Melnikov, M. Münzenberg, U. Nowak, P. M. Oppeneer, R. V. Pisarev, I. Radu, P. van Loosdrecht, as well as to all Ph.D. students and postdoctoral fellows of the Spectroscopy of Solids and Interfaces group for stimulating discussions. This research received funding from Nederlandse Organisatie voor Wetenschappelijk Onderzoek (NWO), Stichting voor Fundamenteel Onderzoek der Materie (FOM), the Dutch Nanotechnology initiative NanoNed, and EC FP7 contributions under Grants No. NMP3-SL-2008-214469 (UltraMagnetron) and No. N 214810 (FANTOMAS).

\section{REFERENCES}

Aeschlimann, M., M. Bauer, S. Pawlik, W. Weber, R. Burgermeister, D. Oberli, and H. C. Siegmann, 1997, Phys. Rev. Lett. 79, 5158.

Aeschlimann, M., A. Vaterlaus, M. Lutz, M. Stampanoni, and 
F. Meier, 1990, J. Appl. Phys. 67, 4438.

Aeschlimann, M., A. Vaterlaus, M. Lutz, M. Stampanoni, F. Meier, H. C. Siegmann, S. Klahn, and P. Hansen, 1991, Appl. Phys. Lett. 59, 2189.

Agranat, M. B., S. I. Ashitkov, A. B. Granovskii, and G. I. Rukman, 1984, Zh. Eksp. Teor. Fiz. 86, 1376 [Sov. Phys. JETP 59, 804 (1984)].

Agranat, M. B., S. I. Ashitkov, A. V. Kirillin, V. E. Fortov, S. I. Anisimov, A. B. Granovskii, and P. S. Kondratenko, 1998, JETP Lett. 67, 953.

Akharoni, A., 2000, Introduction to the Theory of Ferromagnetism (Oxford University Press, New York).

Akhiezer, A. I., V. G. Bar'yakhtar, and S. V. Peletminskii, 1968, Spin Waves (North-Holland, Amsterdam).

Akimoto, R., K. Ando, F. Sasaki, S. Kobayashi, and T. Tani, 1998, J. Appl. Phys. 84, 6318.

Alben, R., E. M. Gyorgy, J. F. Dillon, and J. P. Remeika, 1972, Phys. Rev. B 5, 2560.

Andrade, L. H. F., A. Laraoui, M. Vomir, D. Muller, J.-P. Stoquert, C. Estournés, E. Beaurepaire, and J.-Y. Bigot, 2006, Phys. Rev. Lett. 97, 127401.

Aoyama, J., Y. Hashimoto, and H. Muneka, 2010, J. Appl. Phys. 107, 09C301.

Argyres, P. N., 1955, Phys. Rev. 97, 334.

Astakhov, G. V., H. Hoffmann, V. L. Korenev, T. Kiessling, J. Schwittek, G. M. Schott, C. Gould, W. Ossau, K. Brunner, and L. W. Molenkamp, 2009, Phys. Rev. Lett. 102, 187401.

Astakhov, G. V., A. V. Kimel, G. M. Schott, A. A. Tsvetkov, A. Kirilyuk, D. R. Yakovlev, G. Karczewski, W. Ossau, G. Schmidt, L. W. Molenkamp, and Th. Rasing, 2005, Appl. Phys. Lett. 86, 152506.

Atxitia, U., O. Chubykalo-Fesenko, R. W. Chantrell, U. Nowak, and A. Rebei, 2009, Phys. Rev. Lett. 102, 057203.

Averitt, R. D., A. I. Lobad, C. Kwon, S. A. Trugman, V. K. Thorsmolle, and A. J. Taylor, 2001, Phys. Rev. Lett. 87, 017401.

Awschalom, D. D., J. Warnock, and S. von Molnár, 1987, Phys. Rev. Lett. 58, 812.

Bartelt, A. F., A. Comin, J. Feng, J. R. Nasiatka, T. Eimuller, B. Ludescher, G. Schutz, H. A. Padmore, A. T. Young, and A. Scholl, 2007, Appl. Phys. Lett. 90, 162503.

Beaurepaire, E., J.-C. Merle, A. Daunois, and J.-Y. Bigot, 1996, Phys. Rev. Lett. 76, 4250.

Beaurepaire, E., G. M. Turner, S. M. Harrel, M. C. Beard, J.-Y. Bigot, and C. A. Schmuttenmaer, 2004, Appl. Phys. Lett. 84, 3465.

Belov, K. P., R. A. Volkov, B. P. Goranskii, A. M. Kadomtseva, and V. V. Uskov, 1969, Sov. Phys. Solid State 11, 935.

Bennemann, K. H., 2004, J. Phys.: Condens. Matter 16, R995.

Bergman, B., G. Ju, J. Hohlfeld, R. J. van de Veerdonk, J.-Y. Kim, X. Wu, D. Weller, and B. Koopmans, 2006, Phys. Rev. B 73, 060407.

Berkov, D. V., and N. L. Gorn, 1998, Phys. Rev. B 57, 14332.

Bernal, I., C. W. Struck, and J. G. White, 1963, Acta Crystallogr. 16, 849.

Bhagat, S., and P. Lubitz, 1974, Phys. Rev. B 10, 179.

Bigot, J.-Y., 2001, C. R. Acad. Sci., Ser IV: Phys., Astrophys. 2, 1483.

Bigot, J.-Y., L. Guidoni, E. Beaurepaire, and P. N. Saeta, 2004, Phys. Rev. Lett. 93, 077401.

Bigot, J.-Y., M. Vomir, L. H. F. Andrade, and E. Beaurepaire, 2005, Chem. Phys. 318, 137.

Bigot, J.-Y., M. Vomir, and E. Beaurepaire, 2009, Nat. Phys. 5,
515.

Binder, M., A. Weber, O. Mosendz, G. Woltersdorf, M. Izquierdo, I. Neudecker, J. R. Dahn, T. D. Hatchard, J.-U. Thiele, C. H. Back, and M. R. Scheinfein, 2006, Phys. Rev. B 74, 134404.

Birss, R. R., 1966, Symmetry and Magnetism: Series of Monographs on Selected Topics in Solid State Physics, 2nd ed. (North-Holland, Amsterdam).

Bloch, F., 1930, Z. Phys. 61, 206.

Bloch, F., 1946, Phys. Rev. 70, 460.

Bovensiepen, U., 2006, Appl. Phys. A: Mater. Sci. Process. 82, 395.

Bovensiepen, U., 2007, J. Phys.: Condens. Matter 19, 083201. Carpene, E., E. Mancini, C. Dallera, M. Brenna, E. Puppin, and S. D. Silvestri, 2008, Phys. Rev. B 78, 174422.

Carra, P., B. T. Thole, M. Altarelli, and X. Wang, 1993, Phys. Rev. Lett. 70, 694.

Carva, K., D. Legut, and P. M. Oppeneer, 2009, EPL 86, 57002. Challener, W. A., T. W. McDaniel, C. D. Mihalcea, K. R. Mountfield, K. Pelhos, and I. K. Sendur, 2003, Jpn. J. Appl. Phys. 42, 981.

Chaudhari, P., J. J. Cuomo, and R. J. Gambino, 1973, Appl. Phys. Lett. 22, 337.

Cheskis, D., A. Porat, L. Szapiro, O. Potashnik, and S. Bar-Ad, 2005, Phys. Rev. B 72, 014437.

Chizhik, A. B., I. I. Davidenko, A. Maziewski, and A. Stupakiewicz, 1998, Phys. Rev. B 57, 14366.

Chovan, J., E. G. Kavousanaki, and I. E. Perakis, 2006, Phys. Rev. Lett. 96, 057402.

Chovan, J., and I. E. Perakis, 2008, Phys. Rev. B 77, 085321.

Chubykalo-Fesenko, O., U. Nowak, R. W. Chantrell, and D.

Garanin, 2006, Phys. Rev. B 74, 094436.

Cinchetti, M., M. S. Albaneda, D. Hoffmann, T. Roth, J.-P. Wüstenberg, M. Krauss, O. Andreyev, H. C. Schneider, M. Bauer, and M. Aeschlimann, 2006, Phys. Rev. Lett. 97, 177201.

Comin, A., C. Giannetti, G. Samoggia, P. Vavassori, D. Grando, P. Colombi, E. Bontempi, L. E. Depero, V. Metlushko, B. Ilic, and F. Parmigiani, 2006, Phys. Rev. Lett. 97, 217201.

Cottam, M. G., 1975, J. Phys. C 8, 1933.

Cottam, M. G., and D. J. Lockwood, 1986, Light Scattering in Magnetic Solids (Wiley, New York).

Cywiński, Ł., and L. J. Sham, 2007, Phys. Rev. B 76, 045205.

Dalla Longa, F., J. T. Kohlhepp, W. J. de Jonge, and B. Koopmans, 2007, Phys. Rev. B 75, 224431.

Demokritov, S. O., and B. Hillebrands, 2002, Spin Dynamics in Confined Magnetic Structures I, Topics in Applied Physics Vol. 83 (Springer, Berlin).

Demokritov, S. O., B. Hillebrands, and A. N. Slavin, 2001, Phys. Rep. 348, 441.

Diehl, R., 1975, Solid State Commun. 17, 743.

Dietl, T., H. Ohno, F. Matsukura, J. Cibert, and D. Ferrand, 2000, Science 287, 1019.

Dillon, J. F., E. M. Gyorgy, and J. P. Remeika, 1969, Phys. Rev. Lett. 22, 643.

Djordjevic, M., and M. Münzenberg, 2007, Phys. Rev. B 75, 012404.

Dormann, J. L., F. D’Orazio, F. Lucari, E. Tronc, P. Prené, J. P. Jolivet, D. Fiorani, R. Cherkaoui, and M. Nogués, 1996, Phys. Rev. B 53, 14291.

Duong, N. P., T. Satoh, and M. Fiebig, 2004, Phys. Rev. Lett. 93, 117402 . 
Dzyaloshinskii, I. E., 1957, Sov. Phys. JETP 5, 1259.

Echenique, P. M., R. Berndt, E. V. Chulkov, T. Fauster, A. Goldmann, and U. Höfer, 2004, Surf. Sci. Rep. 52, 219.

Edwards, D. M., and Hertz, J. A., 1973, J. Phys. F: Met. Phys. 3, 2191.

Eilers, G., M. Lüttich, and M. Münzenberg, 2006, Phys. Rev. B 74, 054411.

Eimüller, T., A. Scholl, B. Ludescher, F. Schültz, and J.-U. Thiele, 2007, Appl. Phys. Lett. 91, 042508.

Elliott, R. J., 1954, Phys. Rev. 96, 266.

Eremenko, V. V., S. L. Gnatchenko, I. S. Kachur, V. G. Piryatinskaya, A. M. Ratner, and V. V. Shapiro, 2000, Phys. Rev. B 61, 10670.

Faraday, M., 1846, Philos. Trans. R. Soc. London 136, 104.

Fatti, N. D., C. Voisin, M. Achermann, S. Tzortzakis, D. Christofilos, and F. Vallée, 2000, Phys. Rev. B 61, 16956.

Ferguson, B., and X. Zhang, 2002, Nature Mater. 1, 26.

Ferré, J., and G. A. Gehring, 1984, Rep. Prog. Phys. 47, 513.

Fidler, J., and T. Schrefl, 2000, J. Phys. D 33, R135.

Fiebig, M., N. P. Duong, T. Satoh, B. B. van Aken, K. Miyano, Y. Tomioka, and Y. Tokura, 2008, J. Phys. D 41, 164005.

Fleury, P. A., and R. Loudon, 1968, Phys. Rev. 166, 514.

Furdyna, J. K., and J. Kossut, 1988, Eds., Diluted Magnetic Semiconductors, Semiconductors and Semimetals Vol. 25 (Academic, New York).

Gamble, S. J., M. H. Burkhardt, A. Kashuba, R. Allenspach, S. S. P. Parkin, H. C. Siegmann, and J. Stöhr, 2009, Phys. Rev. Lett. 102, 217201.

Garanin, A., 1991, Physica A 172, 470.

Garanin, A., 1997, Phys. Rev. B 55, 3050.

Gerrits, T., H. A. M. van den Berg, J. Hohlfeld, L. Bär, and Th.

Rasing, 2002, Nature (London) 418, 509.

Gilbert, T. L., 1955, Phys. Rev. 100, 1243.

Gómez-Abal, R., and W. Hübner, 2002, Phys. Rev. B 65, 195114.

Gómez-Abal, R., O. Ney, K. Satitkovitchai, and W. Hübner, 2004, Phys. Rev. Lett. 92, 227402.

Greber, T., T. J. Kreutz, and J. Osterwalder, 1997, Phys. Rev. Lett. 79, 4465.

Gridnev, V. N., 2008, Phys. Rev. B 77, 094426.

Güdde, J., U. Conrad, V. Jähnke, J. Hohlfeld, and E. Matthias, 1999, Phys. Rev. B 59, R6608.

Guidoni, L., E. Beaurepaire, and J.-Y. Bigot, 2002, Phys. Rev. Lett. 89, 017401.

Gupta, J. A., R. Knobel, N. Samarth, and D. D. Awschalom, 2001, Science 292, 2458.

Gurevich, A. G., and G. A. Melkov, 1996, Magnetization Oscillations and Waves (CRC, Boca Raton, FL).

Hall, K. C., J. P. Zahn, A. Gamouras, S. March, J. L. Robb, X. Liu, and J. K. Furdyna, 2008, Appl. Phys. Lett. 93, 032504.

Hansteen, F., A. V. Kimel, A. Kirilyuk, and Th. Rasing, 2005, Phys. Rev. Lett. 95, 047402.

Hansteen, F., A. V. Kimel, A. Kirilyuk, and Th. Rasing, 2006, Phys. Rev. B 73, 014421.

Hashimoto, Y., S. Kobayashi, and H. Munekata, 2008, Phys. Rev. Lett. 100, 067202.

Hashimoto, Y., and H. Munekata, 2008, Appl. Phys. Lett. 93, 202506.

Héroux, J. B., Y. Ino, M. Kuwata-Gonokami, Y. Hashimoto, and S. Katsumoto, 2006, Appl. Phys. Lett. 88, 221110.

Hertel, R., 2006, J. Magn. Magn. Mater. 303, L1.

Hertz, J. A., and D. M. Edwards, 1973, J. Phys. F: Met. Phys. 3, 2174 .
Hicken, R. J., 2003, Philos. Trans. R. Soc. London, Ser. A 361, 2827.

Hillebrands, B., and K. Ounadjela, 2002, Eds., Spin Dynamics in Confined Magnetic Structures I, Topics in Applied Physics (Springer, Berlin).

Hilton, D. J., R. D. Averitt, C. A. Meserole, G. L. Fisher, D. J. Funk, J. D. Thompson, and A. J. Taylor, 2004, Opt. Lett. 29, 1805.

Hilton, D. J., R. P. Prasankumar, S. A. Trugman, A. J. Taylor, and R. D. Averitt, 2006, J. Phys. Soc. Jpn. 75, 011006.

Hohlfeld, J., Th. Gerrits, M. Bilderbeek, Th. Rasing, H. Awano, and N. Ohta, 2001, Phys. Rev. B 65, 012413.

Hohlfeld, J., E. Matthias, R. Knorren, and K. H. Bennemann, 1997, Phys. Rev. Lett. 78, 4861.

Horner, H., and C. M. Varma, 1968, Phys. Rev. Lett. 20, 845. Hübner, W., and G. P. Zhang, 1998, Phys. Rev. B 58, R5920.

Hüfner, S., 1995, Photoelectron Spectroscopy (Springer, Berlin).

Jantz, W., J. R. Sandercock, and W. Wettling, 1976, J. Phys. C 9, 2229.

Ju, G., L. Chen, A. V. Nurmikko, R. F. C. Farrow, R. F. Marks, M. J. Carey, and B. A. Gurney, 2000, Phys. Rev. B 62, 1171. Ju, G., J. Hohlfeld, B. Bergman, R. J. M. van de Veerdonk, O. N. Mryasov, J.-Y. Kim, X. Wu, D. Weller, and B. Koopmans, 2004, Phys. Rev. Lett. 93, 197403.

Ju, G., A. V. Nurmikko, R. F. Farrow, R. F. Marks, M. J. Carey, and B. A. Gurney, 1998, Phys. Rev. B 58, R11857.

Ju, G., A. V. Nurmikko, R. F. C. Farrow, R. F. Marks, M. J. Carey, and B. A. Gurney, 1999, Phys. Rev. Lett. 82, 3705.

Ju, G., A. Vertikov, A. V. Nurmikko, C. Canady, G. Xiao, R. F. C. Farrow, and A. Cebollada, 1998, Phys. Rev. B 57, R700.

Jungwirth, T., J. Sinova, J. Mašek, J. Kučera, and A. H. MacDonald, 2006, Rev. Mod. Phys. 78, 809.

Kabychenkov, A. F., 1991, Zh. Eksp. Teor. Fiz. 100, 1219 [ Sov. Phys. JETP 73, 672 (1991)].

Kahn, F. J., P. S. Pershan, and J. P. Remeika, 1969, Phys. Rev. 186B, 891.

Kalashnikova, A. M., A. V. Kimel, R. V. Pisarev, V. N. Gridnev, A. Kirilyuk, and Th. Rasing, 2007, Phys. Rev. Lett. 99, 167205.

Kalashnikova, A. M., A. V. Kimel, R. V. Pisarev, V. N. Gridnev, P. Usachev, A. Kirilyuk, and Th. Rasing, 2008, Phys. Rev. B 78, 104301.

Kampfrath, T., R. G. Ulbrich, F. Leuenberger, M. Munzenberg, B. Sass, and W. Felsch, 2002, Phys. Rev. B 65, 104429. Kaplan, J., and C. Kittel, 1953, J. Chem. Phys. 21, 760.

Kazantseva, N., D. Hinzke, U. Nowak, R. W. Chantrell, U. Atxitia, and O. Chubykalo-Fesenko, 2008, Phys. Rev. B 77, 184428.

Kazantseva, N., U. Nowak, R. W. Chantrell, J. Hohlfeld, and A. Rebei, 2008, EPL 81, 27004.

Keatley, P. S., V. V. Kruglyak, A. Neudert, E. A. Galaktionov, R. J. Hicken, J. R. Childress, and J. A. Katine, 2008, Phys. Rev. B 78, 214412.

Keller, U., 2003, Nature (London) 424, 831.

Kim, J.-W., K.-D. Lee, J.-W. Jeong, and S.-C. Shin, 2009, Appl. Phys. Lett. 94, 192506.

Kimel, A. V., G. V. Astakhov, G. M. Schott, A. Kirilyuk, D. R. Yakovlev, G. Karczewski, W. Ossau, G. Schmidt, L. W. Molenkamp, and Th. Rasing, 2004, Phys. Rev. Lett. 92, 237203.

Kimel, A. V., F. Bentivegna, V. N. Gridnev, V. V. Pavlov, R. V. Pisarev, and Th. Rasing, 2001, Phys. Rev. B 63, 235201.

Kimel, A. V., B. A. Ivanov, R. V. Pisarev, P. A. Usachev, A. 
Kirilyuk, and Th. Rasing, 2009, Nat. Phys. 5, 727.

Kimel, A. V., A. Kirilyuk, F. Hansteen, R. V. Pisarev, and Th. Rasing, 2007, J. Phys.: Condens. Matter 19, 043201.

Kimel, A. V., A. Kirilyuk, A. Tsvetkov, R. V. Pisarev, and Th. Rasing, 2004, Nature (London) 429, 850.

Kimel, A. V., A. Kirilyuk, P. A. Usachev, R. V. Pisarev, A. M. Balbashov, and Th. Rasing, 2005, Nature (London) 435, 655.

Kimel, A. V., R. V. Pisarev, J. Hohlfeld, and Th. Rasing, 2002, Phys. Rev. Lett. 89, 287401.

Kimel, A. V., C. D. Stanciu, P. A. Usachev, R. V. Pisarev, V. N. Gridnev, A. Kirilyuk, and Th. Rasing, 2006, Phys. Rev. B 74, 060403(R).

Kirilyuk, A., A. V. Kimel, F. Hansteen, R. V. Pisarev, and Th. Rasing, 2006, Low Temp. Phys. 32, 748.

Kirilyuk, A., and Th. Rasing, 2005, J. Opt. Soc. Am. B 22, 148. Kirilyuk, A., Th. Rasing, M. A. M. Haast, and J. C. Lodder, 1998, Appl. Phys. Lett. 72, 2331.

Kise, T., T. Ogasawara, M. Ashida, Y. Tomioka, Y. Tokura, and M. Kuwata-Gonokami, 2000, Phys. Rev. Lett. 85, 1986.

Kisielewski, M., A. Maziewski, V. Zablotskii, and T. Polyakova, 2003, J. Appl. Phys. 93, 6966.

Kittel, C., 1949, Phys. Rev. 76, 743.

Kittel, C., 1958, Phys. Rev. 110, 836.

Kittel, C., 1987, Quantum Theory of Solids (Wiley, New York). Kobayashi, S., Y. Hashimoto, and H. Munekata, 2009, J. Appl. Phys. 105, 07C519.

Kobayashi, T., H. Hayashi, Y. Fujiwara, and S. Shiomi, 2005, IEEE Trans. Magn. 41, 2848.

Kojima, E., R. Shimano, Y. Hashimoto, S. Katsumoto, Y. Iye, and M. Kuwata-Gonokami, 2003, Phys. Rev. B 68, 193203.

Koopmans, B., H. H. J. E. Kicken, M. van Kampen, and W. J. M. de Jonge, 2005, J. Magn. Magn. Mater. 286, 271.

Koopmans, B., G. Malinowski, F. D. Longa, D. Steiauf, M. Fähnle, T. Roth, M. Cinchetti, and M. Aeschlimann, 2010, Nature Mater. 9, 259.

Koopmans, B., J. J. Ruigrok, F. D. Longa, and W. J. de Jonge, 2005, Phys. Rev. Lett. 95, 267207.

Koopmans, B., M. van Kampen, J. T. Kohlhepp, and W. J. M. de Jonge, 2000, Phys. Rev. Lett. 85, 844.

Koshihara, S., A. Oiwa, M. Hirasawa, S. Katsumoto, Y. Iye, C. Urano, H. Takagi, and H. Munekata, 1997, Phys. Rev. Lett. 78, 4617.

Koster, R. G. W., J. O. Dimmock, and H. Statz, 1963, Properties of the Thirty-Two Point Groups (MIT, Cambridge, MA).

Kottler, V., C. Chappert, N. Essaidi, and Y. Chen, 1998, IEEE Trans. Magn. 34, 2012.

Kraft, T., P. M. Oppeneer, V. N. Antonov, and H. Eschrig, 1995, Phys. Rev. B 52, 3561.

Kruglyak, V. V., A. Barman, R. J. Hicken, J. R. Childress, and J. A. Katine, 2005, Phys. Rev. B 71, 220409.

Kruglyak, V. V., R. J. Hicken, M. Ali, B. J. Hickey, A. T. G. Pym, and B. K. Tanner, 2005, Phys. Rev. B 71, 233104.

Kurtzig, A. J., R. Wolfe, R. C. LeCraw, and J. W. Nielsen, 1969, Appl. Phys. Lett. 14, 350.

Landau, L., and E. Lifshitz, 1935, Phys. Z. Sowjetunion 8, 153. Landau, L. D., and E. M. Lifshitz, 1984, Electrodynamics of Continuous Media, Theoretical Physics Vol. 8 (Pergamon, Oxford)

Langner, M. C., C. L. S. Kantner, Y. H. Chu, L. M. Martin, P. Yu, J. Seidel, R. Ramesh, and J. Orenstein, 2009, Phys. Rev. Lett. 102, 177601.

Lefkidis, G., and W. Hübner, 2007, Phys. Rev. B 76, 014418.

Lefkidis, G., G. P. Zhang, and W. Hübner, 2009, Phys. Rev.
Lett. 103, 217401.

Lever, A. B. P., 1984, Inorganic Electronic Spectroscopy (Elsevier Science, Amsterdam).

Li, Y., and K. Baberschke, 1992, Phys. Rev. Lett. 68, 1208.

Lisowski, M., P. Loukakos, U. Bovensiepen, J. Stähler, C. Gahl, and M. Wolf, 2004, Appl. Phys. A: Mater. Sci. Process. 78, 165.

Lisowski, M., P. A. Loukakos, A. Melnikov, I. Radu, L. Ungureanu, M. Wolf, and U. Bovensiepen, 2005, Phys. Rev. Lett. 95, 137402.

Liu, X., Y. Sasaki, and J. K. Furdyna, 2003, Phys. Rev. B 67, 205204.

Lorenc, M., J. Hébert, N. Moisan, E. Trzop, M. Servol, M. B.-L. Cointe, H. Cailleau, M. L. Boillot, E. Pontecorvo, M. Wulff, S. Koshihara, and E. Collet, 2009, Phys. Rev. Lett. 103, 028301.

Mansuripur, M., 1995, The Physical Principles of MagnetoOptical Recording (Cambridge University Press, Cambridge). Mazurenko, D. A., and P. H. M. van Loosdrecht, 2007, private communication.

Meier, F., and B. P. Zakharchenya, 1984, Eds., Optical Orientation, Modern Problems in Condensed Matter Sciences Vol. 8 (North-Holland, Amsterdam).

Meiklejohn, W. H., and C. P. Bean, 1956, Phys. Rev. 102, 1413. Melnikov, A., I. Radu, U. Bovensiepen, O. Krupin, K. Starke, E. Matthias, and M. Wolf, 2003, Phys. Rev. Lett. 91, 227403.

Melnikov, A., I. Radu, A. Polotskiy, T. Wehling, A. Lichtenstein, and U. Bovensiepen, 2008, J. Phys. D: Appl. Phys. 41, 164004.

Metselaar, R., M. A. H. Huyberts, and H. Logmans, 1975, J. Appl. Phys. 46, 3171.

Mitsumori, Y., A. Oiwa, T. Slupinski, H. Maruki, Y. Kashimura, F. Minami, and H. Munekata, 2004, Phys. Rev. B 69, 033203.

Mohn, P., 2003, Magnetism in the Solid State (Springer, Berlin). Morandi, O., P.-A. Hervieux, and G. Manfredi, 2009, Eur. Phys. J. D 52, 155.

Moreau, C. E., I. C. Moraru, N. O. Birge, and W. P. Pratt, 2007, Appl. Phys. Lett. 90, 012101.

Moriya, T., 1960, Phys. Rev. 120, 91.

Müller, G. M., G. Eilers, Z. Wang, M. Scherff, R. Ji, K. Nielsch, C. A. Ross, and M. Münzenberg, 2008, New J. Phys. 10, 123004.

Müller, G. M., et al., 2009, Nature Mater. 8, 56.

Munekata, H., H. Ohno, S. von Molnar, A. Segmüller, L. L. Chang, and L. Esaki, 1989, Phys. Rev. Lett. 63, 1849.

Néel, L., 1949, Ann. Geophys. 5, 99.

Ney, O., M. Trzeciecki, and W. Hübner, 2002, Appl. Phys. B: Lasers Opt. 74, 741.

Nowak, U., O. N. Mryasov, R. Wieser, K. Guslienko, and R. W. Chantrell, 2005, Phys. Rev. B 72, 172410.

Nye, J. F., 2004, Physical Properties of Crystals (Clarendon, Oxford).

O'Dell, T. H., 1970, The Electrodynamics of Magneto-Electric Media (North-Holland, Amsterdam).

Ogasawara, T., N. Iwata, Y. Murakami, H. Okamoto, and Y. Tokura, 2009, Appl. Phys. Lett. 94, 162507.

Ogasawara, T., K. Ohgushi, Y. Tomioka, K. S. Takahashi, H. Okamoto, M. Kawasaki, and Y. Tokura, 2005, Phys. Rev. Lett. 94, 087202.

Ohno, H., H. Munekata, T. Penney, S. von Molnár, and L. L. Chang, 1992, Phys. Rev. Lett. 68, 2664.

Oiwa, A., Y. Mitsumori, R. Moriya, T. Slupinski, and H. Mu- 
nekata, 2002, Phys. Rev. Lett. 88, 137202.

Oppeneer, P. M., and A. Liebsch, 2004, J. Phys.: Condens. Matter 16, 5519.

Paoletti, A., 1978, Ed., Physics of Magnetic Garnets, Enrico Fermi International School of Physics, Italian Physical Society (North-Holland, Amsterdam).

Perroni, C. A., and A. Liebsch, 2006, Phys. Rev. B 74, 134430.

Pershan, P. S., 1963, Phys. Rev. 130, 919.

Pershan, P. S., J. P. van der Ziel, and L. D. Malmstrom, 1966, Phys. Rev. 143, 574.

Pitaevskii, L. P., 1961, Sov. Phys. JETP 12, 1008.

Qi, J., Y. Xu, A. Steigerwald, X. Liu, J. K. Furdina, I. Perakis, and N. H. Tolk, 2009, Phys. Rev. B 79, 085304.

Qiu, Z., and S. Bader, 1999, J. Magn. Magn. Mater. 200, 664.

Radu, I., G. Woltersdorf, M. Kiessling, A. Melnikov, U. Bovensiepen, J.-U. Theile, and C. H. Back, 2009, Phys. Rev. Lett. 102, 117201.

Randoshkin, V. V., V. A. Polezhaev, N. N. Sysoev, and Y. N. Sazhin, 2003, Phys. Solid State 45, 513.

Regensburger, H., R. Vollmer, and J. Kirschner, 2000, Phys. Rev. B 61, 14716.

Respaud, M., M. Goiran, J. M. Broto, F. H. Yang, T. O. Ely, C. Amiens, and B. Chaudret, 1999, Phys. Rev. B 59, R3934.

Rhie, H. S., H. A. Dürr, and W. Eberhardt, 2003, Phys. Rev. Lett. 90, 247201.

Roth, T., D. Steil, D. Hoffmann, M. Bauer, M. Cinchetti, and M. Aeschlimann, 2008, J. Phys. D 41, 164001.

Rozkotová, E., P. Němec, P. Horodyská, D. Spinzl, F. Trojánek, P. Malý, V. Novák, K. Olejník, M. Cukr, and T. Jungwirth, 2008a, Appl. Phys. Lett. 92, 122507.

Rozkotová, E., P. Němec, P. Horodyská, D. Spinzl, F. Trojánek, P. Malý, V. Novák, K. Olejník, M. Cukr, and T. Jungwirth, 2008b, IEEE Trans. Magn. 44, 2674.

Rzhevsky, A. A., B. B. Krichevtsov, D. E. Bürgler, and C. M. Schneider, 2007, Phys. Rev. B 75, 224434.

Satoh, T., B. B. V. Aken, N. P. Duong, T. Lottermoser, and M. Fiebig, 2007, J. Magn. Magn. Mater. 310, 1604.

Satoh, T., N. P. Duong, and M. Fiebig, 2006, Phys. Rev. B 74, 012404.

Schmidt, A. B., M. Pickel, M. Wiemhöfer, M. Donath, and M. Weinelt, 2005, Phys. Rev. Lett. 95, 107402.

Schneider, C. M., K. Holldack, M. Kinzler, M. Grunze, H. P. Oepen, F. Schaers, H. Petersen, K. Meinel, and J. Kirschner, 1993, Appl. Phys. Lett. 63, 2432.

Schober, J., 1976, IEEE Trans. Magn. 12, 401.

Scholl, A., L. Baumgarten, R. Jacquemin, and W. Eberhardt, 1997, Phys. Rev. Lett. 79, 5146.

Schumacher, H. W., C. Chappert, P. Crozat, R. C. Sousa, P. P. Freitas, J. Miltat, J. Fassbender, and B. Hillebrands, 2003, Phys. Rev. Lett. 90, 017201.

Schütz, G., W. Wagner, W. Wilhelm, P. Kienle, R. Zeller, R. Frahm, and G. Materlik, 1987, Phys. Rev. Lett. 58, 737.

Shah, J., 1996, Ultrafast Spectroscopy of Semiconductors and Semiconductor Nanostructures, Springer Series in Solid-State Sciences Vol. 115 (Springer, Berlin).

Sham, L. J., 1999, J. Magn. Magn. Mater. 200, 219.

Shapiro, S. M., J. D. Axe, and J. P. Remeika, 1974, Phys. Rev. B 10, 2014.

Shen, Y. R., 1984, The Principles of Nonlinear Optics (Wiley, New York).

Shen, Y. R., and N. Bloembergen, 1966, Phys. Rev. 143, 372.

Silva, T., J. Nibarger, B. Rippard, and B. Stamps, 2004, Bull. Am. Phys. Soc. 49, B23011.
Smolenskǐ̆, G. A., R. V. Pisarev, and I. G. Sinii, 1975, Sov. Phys. Usp. 18, 410.

Stamm, C., T. Kachel, N. Pontius, R. Mitzner, T. Quast, K. Holldack, S. Khan, C. Lupulescu, E. F. Aziz, M. Wietstruk, H. A. Dürr, and W. Eberhardt, 2007, Nature Mater. 6, 740.

Stanciu, C., A. Tsukamoto, A. V. Kimel, F. Hansteen, A. Kirilyuk, A. Itoh, and Th. Rasing, 2007, Phys. Rev. Lett. 99, 217204.

Stanciu, C. D., F. Hansteen, A. V. Kimel, A. Tsukamoto, A. Itoh, A. Kirilyuk, and Th. Rasing, 2007, Phys. Rev. Lett. 99, 047401.

Stanciu, C. D., A. V. Kimel, F. Hansteen, A. Tsukamoto, A. Itoh, A. Kirilyuk, and Th. Rasing, 2006, Phys. Rev. B 73, 220402(R).

Steiauf, D., and M. Fähnle, 2009, Phys. Rev. B 79, 140401.

Stevens, T. E., J. Kuhl, and R. Merlin, 2002, Phys. Rev. B 65, 144304.

Stöhr, J., and H. C. Siegmann, 2006, Magnetism: From Fundamentals to Nanoscale Dynamics (Springer, Berlin).

Stöhr, J., Y. Wu, B. D. Hermsmeir, M. G. Samant, G. R. Harp, S. Koranda, D. Dunham, and B. P. Tonner, 1993, Science 259, 658.

Stupakiewicz, A., A. Maziewski, I. Davidenko, and V. Zablotskii, 2001, Phys. Rev. B 64, 064405.

Tanabe, Y., and K. Aoyagi, 1982, Excitons (North-Holland, Amsterdam), Vol. 2.

Teale, R. W., and D. W. Temple, 1967, Phys. Rev. Lett. 19, 904.

Thiele, J.-U., M. Buess, and C. H. Back, 2004, Appl. Phys. Lett. 85, 2857.

Thiele, J.-U., S. Maat, and E. E. Fullerton, 2003, Appl. Phys. Lett. 82, 2859.

Tudosa, I., C. Stamm, A. B. Kashuba, F. King, H. C. Siegmann, J. Stöhr, G. Ju, B. Lu, and D. Weller, 2004, Nature (London) 428, 831.

Vahaplar, K., A. M. Kalashnikova, A. V. Kimel, D. Hinzke, U. Nowak, R. Chantrell, A. Tsukamoto, A. Itoh, A. Kirilyuk, and Th. Rasing, 2009, Phys. Rev. Lett. 103, 117201.

van der Laan, G., B. T. Thole, G. A. Sawatzky, J. B. Goedkoop, J. C. Fuggle, J.-M. Esteva, R. Karnatak, J. P. Remeika, and H. A. Dabkowska, 1986, Phys. Rev. B 34, 6529.

van der Ziel, J. P., P. S. Pershan, and L. D. Malmstrom, 1965, Phys. Rev. Lett. 15, 190.

van Kampen, M., C. Jozsa, J. T. Kohlhepp, P. LeClair, L. Lagae, W. J. M. de Jonge, and B. Koopmans, 2002, Phys. Rev. Lett. 88, 227201.

Vaterlaus, A., T. Beutler, and F. Meier, 1990, J. Appl. Phys. 67, 5661.

Vaterlaus, A., T. Beutler, and F. Meier, 1991, Phys. Rev. Lett. 67, 3314.

Vernes, A., and P. Weinberger, 2005, Phys. Rev. B 71, 165108. Versluis, J. H., A. V. Kimel, V. N. Gridnev, D. R. Yakovlev, G. Karczewski, T. Wojtowicz, J. Kossut, A. Kirilyuk, and Th. Rasing, 2009, Phys. Rev. B 80, 235326.

Veselago, V. G., R. A. Doroshenko, and S. G. Rudov, 1994, Zh. Eksp. Teor. Fiz. 105, 638 [ JETP 78, 341 (1994)].

Vomir, M., L. H. F. Andrade, L. Guidoni, E. Beaurepaire, and J.-Y. Bigot, 2005, Phys. Rev. Lett. 94, 237601.

Walowski, J., G. Müller, M. Djordjevic, M. Münzenberg, M. Kläui, C. A. F. Vaz, and J. A. C. Bland, 2008, Phys. Rev. Lett. 101, 237401.

Wang, D. M., Y. H. Ren, X. Liu, J. K. Furdyna, M. Grimsditch, and R. Merlin, 2007, Phys. Rev. B 75, 233308.

Wang, J., I. Cotoros, D. S. Chemla, X. Liu, J. K. Furdyna, J. 
Chovan, and I. E. Perakis, 2009, Appl. Phys. Lett. 94, 021101.

Wang, J., I. Cotoros, K. M. Dani, X. Liu, J. K. Furdyna, and D. S. Chemla, 2007, Phys. Rev. Lett. 98, 217401.

Wang, J., L. Cywinski, C. Sun, J. Kono, H. Munekata, and L. J. Sham, 2008, Phys. Rev. B 77, 235308.

Wang, J., C. Sun, Y. Hashimoto, J. Kono, G. A. Khodaparast, L. Cywiński, L. J. Sham, G. D. Sanders, C. J. Stanton, and H. Munekata, 2006, J. Phys.: Condens. Matter 18, R501.

Wang, J., C. Sun, J. Kono, A. Oiwa, H. Munekata, Ł. Cywiński, and L. J. Sham, 2005, Phys. Rev. Lett. 95, 167401.

Wangsness, R. K., 1953, Phys. Rev. 91, 1085.

Wangsness, R. K., 1954, Phys. Rev. 93, 68.

Wangsness, R. K., 1955, Phys. Rev. 97, 831.

Weber, M. C., H. Nembach, and J. Fassbender, 2004, J. Appl. Phys. 95, 6613.

Weng, R. S., and M. H. Kryder, 1993, IEEE Trans. Magn. 29, 2177.

White, R. L., 1969, J. Appl. Phys. 40, 1061.

White, R. M., R. J. Nemanich, and C. Herring, 1982, Phys. Rev. B 25, 1822.

Wijn, H. P. J., 1994, Numerical Data and Functional Relationships, Landolt-Börnstein, New Series, Group III, Vol. $27 \mathrm{f}$ (Springer, Berlin).

Wilks, R., R. J. Hicken, M. Ali, and B. J. Hickey, 2005, J. Appl. Phys. 97, 10A705.

Wilks, R., R. J. Hicken, M. Ali, B. J. Hickey, J. D. R. Buchanan, A. T. G. Pym, and B. K. Tanner, 2004, J. Appl. Phys. 95, 7441.

Woodford, S. R., 2009, Phys. Rev. B 79, 212412.

Woodford, S. R., A. Bringer, and S. Blügel, 2007, J. Appl. Phys. 101, 053912.

Wu, T., 1997, J. Appl. Phys. 81, 5321.

Xu, Z., X. D. Liu, R. X. Gao, Z. F. Chen, T. S. Lai, N. H. Hu,
S. M. Zhou, X. J. Bai, and J. Du, 2008, Appl. Phys. Lett. 93, 162509.

Yafet, Y., 1963, Solid State Physics (Academic, New York), Vol. 14.

Yan, Y.-X., E. B. Gamble, and K. A. Nelson, 1985, J. Chem. Phys. 83, 5391.

Zakharov, V. E., and E. A. Kuznetsov, 1984, in Soviet Scientific Reviews, Section C: Mathematical Physics Reviews, edited by S. P. Novikov (OPA, Amsterdam).

Zhan, H., J. Deibel, J. Laib, C. Sun, J. Kono, D. Mittleman, and H. Munekata, 2007, Appl. Phys. Lett. 90, 012103.

Zhang, G., W. Hübner, E. Beaurepaire, and J.-Y. Bigot, 2002, Spin Dynamics in Confined Magnetic Structures I, Topics in Applied Physics Vol. 83 (Springer, Berlin).

Zhang, G. P., and T. George, 2008, Phys. Rev. B 78, 052407.

Zhang, G. P., and W. Hübner, 2000, Phys. Rev. Lett. 85, 3025.

Zhang, G. P., W. Hübner, G. Lefkidis, Y. Bai, and T. F. George, 2009, Nat. Phys. 5, 499.

Zhang, Q., A. V. Nurmikko, A. Anguelouch, G. Xiao, and A. Gupta, 2002, Phys. Rev. Lett. 89, 177402.

Zhang, Q., A. V. Nurmikko, G. X. Miao, G. Xiao, and A. Gupta, 2006, Phys. Rev. B 74, 064414.

Zhao, J., A. V. Bragas, D. J. Lockwood, and R. Merlin, 2004, Phys. Rev. Lett. 93, 107203.

Zhu, Y., X. Zhang, T. Li, L. Chen, J. Lu, and J. Zhao, 2009, Appl. Phys. Lett. 94, 142109.

Zhukov, V. P., and E. V. Chulkov, 2009, Phys. Usp. 52, 105.

Zon, B. A., and V. Y. Kupershmidt, 1983, Sov. Phys. Solid State 25, 708.

Žutić, I., J. Fabian, and S. Das Sarma, 2004, Rev. Mod. Phys. 76, 323.

Zvezdin, A. K., and V. A. Kotov, 1997, Modern Magnetooptics and Magnetooptical Materials (IOP, Bristol). 